\title{
Quantum Magnetism, Nonequilibrium Dynamics and Quantum Simulation of Correlated Quantum Systems
}

Kumulative Habilitationsschrift VORGELEGT VON

DR. RER. NAT. DiPl.-Phys.

Salvatore Rosario Manmana aus Catania (Italien)

Institut für Theoretische Physik

Fakultät für Physik

Georg-August-Universität Göttingen 


\section{Contents}

Deutsche Zusammenfassung . . . . . . . . . . . . . . . v

English Short Summary . . . . . . . . . . . . . vi

I Introduction $\quad 1$

1 Quantum Many Body Systems 5

2 MPS and Further Developments of DMRG 13

2.1 MPS and Matrix Product Operators (MPO) . . . . . . . . . 14

2.2 Projected Entangled Pair States (PEPS) . . . . . . . . . . 21

2.3 Time Evolution with the Adaptive t-DMRG . . . . . . . . 23

3 Quantum Magnets 27

3.1 Unconventional Phases and Phase Transitions . . . . . . . . . 27

3.2 Effect of Spin-Orbit Coupling . . . . . . . . . . . . . 31

4 Quantum Simulation 35

4.1 Realizations of SU(N) Symmetric Systems . . . . . . . . . 36

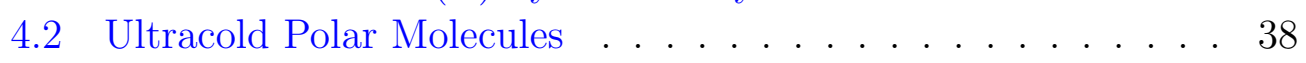

4.3 Topological Phases . . . . . . . . . . . . . . . . . . . . . . 41

5 Nonequilibrium Dynamics 45

5.1 Relaxation after Quantum Quenches . . . . . . . . . . . 47

5.2 Transport on Optical Lattices . . . . . . . . . . . . . . 51

6 Outlook $\quad 55$

List of Own Contributions $\quad 57$ 
II Selected Results Published in Refereed Journals 65

7 Quantum Critical Behavior $\quad 69$

7.1 Spin Systems In Magnetic Fields . . . . . . . . . . . . 70

7.2 Quantum Magnets with Spin-Orbit Coupling . . . . . . . . . 75

7.3 Quantum Information Approach . . . . . . . . . . . . . . 79

8 Quantum Simulation $\quad 83$

9 Non-Equilibrium Dynamics $\quad 89$

9.1 Quantum Quenches . . . . . . . . . . . . . . . . . . . 90

9.2 Transport on Optical Lattices . . . . . . . . . . . . . . . 93

$\begin{array}{ll}\text { Acknowledgements } & 97\end{array}$

$\begin{array}{ll}\text { Bibliography } & 99\end{array}$ 


\section{Deutsche Zusammenfassung}

Die theoretische und experimentelle Untersuchung stark korrelierter Quantensysteme im und ausserhalb des Gleichgewichts ist in den letzten Jahren zu einem zentralen Forschungsschwerpunkt in der Physik der kondensierten Materie geworden. In dieser Habilitationsschrift werden anhand eigener Beiträge aktuelle Entwicklungen diskutiert und ein Ausblick auf zukünftige Entwicklungen des Feldes gegeben. Es wird dabei ein direkter Bezug von theoretischen Zugängen zu aktuellen Experimenten mit ultrakalten Gasen und mit stark korrelierten Materialien genommen. Insbesondere werden mikroskopische Modelle diskutiert, die zur Modellierung stark korrelierter Materialien (wie z.B. Quantenmagnete) herangezogen werden. Das zweite Thema der Schrift behandelt die Realisierung und Untersuchung von Quantenvielteilcheneffekten in aktuellen Experimenten mit ultrakalten Gasen durch sogenannte Quantensimulatoren. Ziel ist es, aufgrund theoretischer Überlegungen Vorhersagen zur Emulation stark korrelierter Systeme zu treffen und, basierend auf numerisch exakten Simulationen, Vorhersagen für die Experimente zu treffen. Da solche Experimente insbesondere auch ausserhalb des Gleichgewichts durchgeführt werden können, knüpft dies direkt an das dritte Thema der Schrift an, in dem die Physik von Quantenvielteilchensystemen ausserhalb des Gleichgewichts untersucht wird. Dazu gehören die Behandlung des Relaxationsverhaltens nach sogenannten Quantum Quenches und Fragestellungen zum Transport, die hier anhand von Situationen auf optischen Gittern diskutiert werden. Die drei Themenbereiche werden überspannt von der Fragestellung, wie man neuartige Quantenzustände realisieren kann, und wie man diese charakterisieren kann. Ausserhalb des Gleichgewichts erwächst die Fragestellung, ob durch das Relaxationsverhalten ungewöhnliche Zustände erzeugt werden können. Wie in dieser Arbeit diskutiert, ergeben sich in der Tat unerwartete Ergebnisse, wie die Bildung negativer Temperaturen, oder das Anwachsen der Teilchendichte verursacht durch repulsive Wechselwirkungen. Im Gleichgewicht kann das Vorhandensein starker Magnetfelder zu interessantem Verhalten führen, wie anhand verschiedener Systeme diskutiert wird. Hervorzuheben sind hier die Bildung unkonventioneller Mott-Isolatoren, von Phasen mit 'spin-nematischer' Ordnung, und unkonventionelle Quantenphasenübergänge. Eine aktuelle Entwicklung der aktuellen Forschung behandelt die Bildung sogenannter topologischer Phasen, die nicht durch einen lokalen Ordnungsparameter beschrieben werden und sich somit der Charakterisierung im Rahmen der LandauTheorie von Phasen und Phasenübergängen entziehen. Aktuelle Fragestellungen sind die Realisierung solcher Phasen im Vorhandensein von Wech- 
selwirkungen, auch langreichweitiger Natur, wie sie in Quantensimulatoren mit polaren Molekülen in Erscheinung treten. In dieser Schrift wird diskutiert, wie diese Fragestellungen durch einen numerischen Zugang - die Dichtematrixrenormierungsgruppe - behandelt werden können und wie diese sehr flexible Methode zu interessanten Einsichten in die facettenreiche Physik stark korrelierter Quantensysteme führt.

\section{English Short Summary}

The theoretical and experimental investigation of strongly correlated quantum systems in- and out-of-equilibrium has been a recent focus of research in condensed matter physics. In this Habilitation thesis, I present recent and future developments of the field in the context of my own contributions. A direct relation between theoretical approaches and experiments with ultracold gases and in strongly correlated materials is made, and I discuss useful microscopic models for the description of such materials (e.g., quantum magnets). The second topic of the thesis addresses the realization of quantum many body effects in ongoing experiments with ultracold gases in the context of so-called 'Quantum Simulators'. Theoretical considerations based on so-called 'numerically exact' simulations are used to make predictions for the concrete experimental implementation of such emulators for strongly correlated systems. These experiments can be performed in a very well controlled way out-of-equilibrium, which directly connects to the third topic of the thesis, the investigation of the nonequilibrium dynamics of quantum many body systems. This addresses the relaxation behavior after so-called 'Quantum Quenches' and problems related to transport, here discussed in the context of optical lattices. The three topics are bridged by the question for the realization and characterization of novel quantum states of matter. In out-of-equilibrium situations the question is addressed if such states can be identified in the relaxation behavior. As further discussed in this thesis, indeed unconventional behavior as, e.g., the formation of 'absolute negative temperatures' and the increase of particle population in the presence of repulsive interactions is realized. In equilibrium, a bouquet of interesting behavior in various systems can be revealed in the presence of strong magnetic fields. Examples are unconventional Mott insulators, phases with 'spin-nematic order' and unusual quantum critical behavior. A recent development addresses so-called topological phases which are not described in terms of a local order parameter and hence do not fall into the realm of validity of the usual Landau theory of phases and phase transitions. Questions arise for the effect 
of interactions, in particular also the presence of long-range interactions as realized in quantum simulators with ultracold polar molecules. The thesis demonstrates how these aspects can be addressed via numerical methods in particular the density matrix renormalization group method - and how this approach has been leading to interesting insights into the multi-facetted behavior of correlated quantum systems. 


\section{Part I}

\section{Introduction}



This thesis is devoted to the theoretical investigation of strongly correlated quantum systems in- and out-of-equilibrium. In the first part, I introduce the field, the methods and various topics of present day research. In the second part of the thesis, several of my own contributions are presented which cover the various topics discussed in the first part.

After a short introduction to quantum many body systems in Ch. 1, I introduce the numerical methods relevant for the thesis in Ch. 2. According to my own research, and to relevant topics in present day research activities, it is followed by three chapters discussing various realizations and aspects of quantum many body systems. In Ch. 3, quantum critical behavior in the context of strongly correlated materials, in particular quantum magnets in strong magnetic fields, is treated. Ch. 4 discusses ongoing and future experiments with ultracold gases on optical lattices, so-called quantum simulators, and their theoretical description. In particular, systems of alkaline earth atoms and polar molecules are treated. These systems lead to the implementation of $\mathrm{SU}(\mathrm{N})$ symmetric models or to the emulation of quantum magnetism and superconductivity on optical lattices. An important aspect of these investigations is the non-equilibrium behavior in the experiments, which will be addressed in Ch. 5. In this context, various non-equilibrium systems are treated which are topics of present day research, in particular quantum quenches and transport in the presence of interactions. In Ch. 6, I summarize the first part and give a short outlook to further developments in the field. 


\section{Chapter 1}

\section{Quantum Many Body Systems}

Quantum many body effects come into play in certain materials and in systems of ultracold atomic and molecular gases on optical lattices [1,2]. Prominent examples for strongly correlated materials are high-temperature superconductors [3, 4] and frustrated quantum magnets [5-7]. Using ultracold atoms, a breakthrough experiment was the realization of a Mott-insulating state of ultracold bosons in 2002 [8]. These systems are described by microscopic quantum mechanical models of interacting particles on various lattice geometries. Interesting effects arise due to competing interactions or geometrical frustration which typically does not allow for the realization of a simple ground state that satisfies all bonds equally well. Examples for such frustrated geometries of interest in ongoing research are shown in Fig. 1.1. Due to these competing interactions, novel and interesting states of matter can be realized [5-7]. Ongoing theoretical and experimental research efforts are concerned with identifying and characterizing such new states, and a large part of this thesis is devoted to the finding of such unconventional phases in the context of strongly correlated materials and in quantum simulators. In the following, own contributions are put in the perspective of ongoing research activities in the field.

An interesting class of strongly correlated materials are frustrated quantum magnets [5-7]. These systems can be described as networks of interacting quantum mechanical spins on a lattice, and the underlying microscopic model 

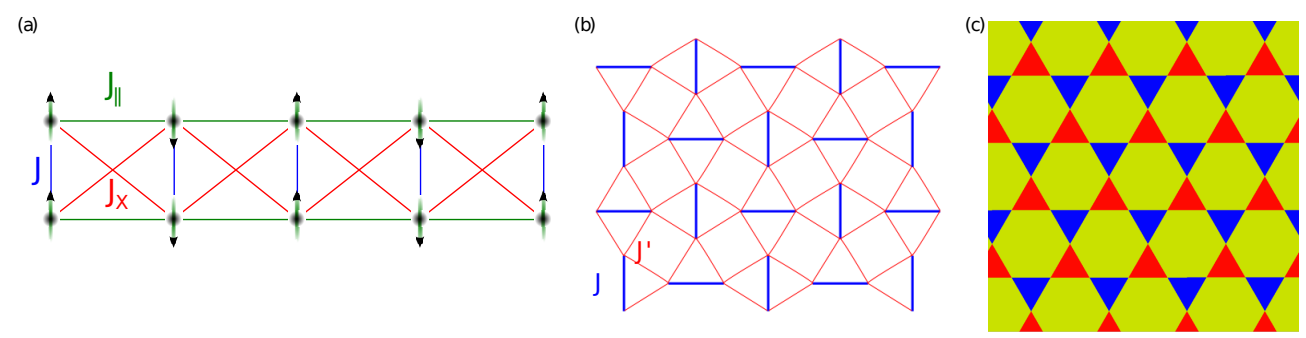

Figure 1.1: Examples of frustrated lattice geometries realized in quantum magnetic materials. (a) Frustrated ladder, realized in various materials, e.g., $\mathrm{TlCuCl}_{3}[9,10]$. (b) Shastry-Sutherland lattice, a network of orthogonal dimers. This geometry is realized, e.g., in $\mathrm{SrCu}_{2}\left(\mathrm{BO}_{3}\right)_{2}[11,12]$. (c) Example of a kagome lattice of corner-sharing triangles. This system is realized, e.g., in Herbertsmithite $\left[\mathrm{ZnCu}_{3}(\mathrm{OH})_{6} \mathrm{Cl}_{2}\right]$ [13] (figure from Wikipedia).

is the Heisenberg Hamiltonian,

$$
\mathcal{H}_{\text {Heisenberg }}=\sum_{\langle i, j\rangle} J_{i, j} \vec{S}_{i} \cdot \vec{S}_{j}
$$

where the spins $\vec{S}$ are located on the lattice sites $i$ and $j$. In principle, any magnitude of $S \equiv|\vec{S}|$ can be realized, but the most appealing effects due to the quantum nature of the spins are expected for small values of the spin, e.g., $S=1 / 2$ or $S=1$. These materials host a rich bouquet of interesting phenomena which are revealed in the presence of an external magnetic field. Particularly intriguing is the realization of unconventional quantum states of matter, as e.g., a Bose-Einstein-condensate (BEC) of triplet excitations [10, 14-16], Mott-insulators on magnetization plateaux [11, 12, 17, 18], and the proposed spin-equivalent [19-24] of a supersolid phase [25-29] in their vicinity which is characterized by the spontaneous breaking of the translational symmetry of the underlying lattice which happens simultaneously with the breaking of a $\mathrm{U}(1)$ symmetry associated to the formation of a superfluid. These effects most prominently appear at low temperatures at which quantum fluctuations dominate over thermodynamic fluctuations, and which drive quantum phase transitions in the phase diagrams of these systems [30, 31].

In numerical approaches, the spontaneous breaking of the symmetries of the system and the stabilization of a phase are typically investigated by computing local observables and correlation functions on finite systems and extrapolating these results to the thermodynamic limit. This, however, often leaves the question open of whether the relevant correlation functions have 
been looked at. This is addressed in recent developments by computing the so-called correlation density matrices, in which appropriate statistical operators and their spectral decomposition are analyzed [32-34]. The eigenvectors of these correlation density matrices are then associated to correlation functions, the most important ones having the largest eigenvalue. As an example for the usefulness of this approach, see my contribution [SRM28] (attached in the second part of the thesis) in which a similar approach is used for the identification of quantum criticality in a $2 \mathrm{D}$ kagome system for which only small systems could be treated. In 1D, gapless phases and the universality class of transitions can be characterized by the central charge $c$ in the context of conformal field theories (CFT) $[35,36]$. As demonstrated by Vidal et al. [37] and by Calabrese and Cardy [38], this quantity can easily be obtained by computing the von Neumann entanglement entropy

$$
S_{\ell}=-\operatorname{Tr}\left[\varrho_{\ell} \ln \left(\varrho_{\ell}\right)\right],
$$

with $\varrho_{\ell}$ the reduced density matrix of a subsystem of size $\ell$. For critical 1D systems of size $L$ with periodic boundary conditions and a subsystem of size $\ell$, the relation

$$
S_{\ell}=\frac{c}{3} \ln \left[\frac{L}{\pi} \sin \left(\frac{\pi \ell}{L}\right)\right]+g_{\mathrm{PBC}}
$$

is obtained, with $g_{\mathrm{PBC}}$ a constant independent of $c$. Thus, computing $S_{\ell}$ numerically allows one to extract the value of $c$ rather easily. This can be done using the density matrix renormalization group method (DMRG) [39, 40] which will be discussed in more detail below. This approach is now widely used to investigate quantum criticality in 1D systems, and together with the direct computation of observables it constitutes a powerful toolbox for the investigation of low-dimensional correlated quantum systems.

Other developments adopt concepts from quantum information theory [41] to investigate quantum critical behavior. One of these proposes to use the overlap between ground states in different phases (often referred to as fidelity) [42] or the fidelity susceptibility (also called fidelity metric) [43, 44] which for the ground state $\left|\psi_{0}\right\rangle$ is obtained for a certain value of some control parameter $U$ as

$$
\chi(U)=\frac{2\left[1-\left|\left\langle\psi_{0}(U) \mid \psi_{0}(U+d U)\right\rangle\right|\right]}{L d U^{2}} .
$$

The underlying picture is that at a phase transition the nature of the wave function changes drastically, so that in the thermodynamic limit a divergence of $\chi$ should appear at the critical point. The hope is that such a behavior can be seen for small systems already, so that computing $\chi$ should facilitate the investigation of systems using standard numerical approaches. Indeed, 
for various systems, this has been seen (see, e.g., [45-49] and my own contribution [SRM9]). However, a field-theoretical analysis indicates that the singular part of $\chi$ does not necessarily diverge in the thermodynamic limit, indicating that for some systems it will be difficult to characterize quantum criticality solely relying on the behavior of $\chi[44]$. Furthermore, as discussed in the second part of this thesis, my own contributions show that there are systems in which the finite-size scaling is difficult, and large system sizes as well as a high accuracy are required [SRM20, SRM22] (see also [50]). Therefore, caution is needed when applying this approach.

Special attention is devoted to phases stabilized by non-obvious spontaneous breaking of symmetries of the Hamiltonian. A prominent example is realized for $S>1 / 2$. In this case, the $\mathrm{SU}(2)$ symmetry of the Heisenberg Hamiltonian can spontaneously be broken without resulting finite local magnetizations, realizing a rather unconventional ordered phase which would appear disordered under a conventional perspective in which only local magnetizations are considered. Such a state can be related to liquid crystals which realize nematic states with a broken spin-rotational symmetry but unbroken time reversal symmetry [7, 51-60]. Correspondingly, such states are called spinnematic states and have been explored in a number of theoretical approaches, e.g. [61-64] and my own contribution [SRM25] (attached in the second part of the thesis; a nice summary of the state of the art is sketched in the introduction of [60] and references therein). An example for a quantum magnetic material which might realize such a phase is $\mathrm{NiGa}_{2} \mathrm{~S}_{4}$ [65], a $S=1$ spin system on a triangular lattice. However, a convincing experimental proof for the existence of such a phase is still missing, and it would be interesting to identify it either in quantum magnetic materials or in experiments on optical lattices. This might be achieved in quantum simulators for $\mathrm{SU}(\mathrm{N})$ systems using alkaline earth atoms [66] which are further discussed below.

I want to briefly mention that there are phases in which, despite the presence of strong correlations in the system, no long-range order is induced at zero temperature. These phases are called spin liquids and can be pictured as a superposition of many spins which simultaneously point in different directions. They show exotic behavior and possess a number of interesting properties such as excitations with fractional quantum numbers [67]. There exists a vast literature on this topic, for an introduction see [68]. The search for realizations of this type of unconventional states is motivating a lot of ongoing research. Recently, numerical methods have provided evidence for the existence of such a spin liquid phase in the kagome lattice [69-71], which is depicted in Fig. 1.1 and which has been identified in the natural mineral Herbertsmithite $[13,72]$. This is therefore an interesting example for how a 
numerical study can uncover interesting phases of matter which may find a realization in nature in strongly correlated materials. However, since these phases are not central for this thesis, I refrain at this point from a more detailed treatment and refer to the literature.

While the interacting spins in the Heisenberg model remain localized, in many materials the electrons are itinerant. This is addressed, e.g., by the Hubbard model [73-76], which is one of the simplest models taking into account the effects of spin and of electron motion. Both, the fermionic version, ${ }^{1}$

$$
\mathcal{H}_{\text {Hubbard }}^{\text {Fermions }}=-t \sum_{\substack{\langle i, j\rangle \\ \sigma}}\left[c_{i, \sigma}^{\dagger} c_{j, \sigma}+\text { h.c. }\right]+U \sum_{i} n_{i, \uparrow} n_{i, \downarrow}
$$

as well as the bosonic variant [30, 77],

$$
\mathcal{H}_{\text {Hubbard }}^{\text {Bosons }}=-J \sum_{\langle i, j\rangle}\left[b_{i}^{\dagger} b_{j}+\text { h.c. }\right]+\frac{U}{2} \sum_{i} n_{i}\left(n_{i}-1\right)
$$

are relevant for the description of strongly correlated materials or for systems of ultracold atoms on optical lattices, respectively. Due to the difficulties to treat in particular the fermionic variant of this system beyond 1D using analytical or numerical approaches, the experiments on optical lattices have got the particular motivation to emulate the behavior of this microscopic model, so that its phase diagram can be investigated in such experiments. This is in the spirit of Feynman's proposal from the early 1980 s $^{2}$ to use some well controlled quantum systems to simulate other ones, eventually leading to the development of a quantum computer [79-81]. There are new developments aiming at the emulation of the Heisenberg and the $\mathrm{t}-\mathrm{J}$-model

$$
\mathcal{H}_{\mathrm{tJ}}=-t \sum_{\substack{\langle i, j\rangle \\ \sigma}}\left(f_{i, \sigma}^{\dagger} f_{j, \sigma}+\text { h.c. }\right)+J \sum_{\langle i, j\rangle}\left(\vec{S}_{i} \cdot \vec{S}_{j}-\frac{1}{4} n_{i} n_{j}\right),
$$

which can be obtained from the fermionic Hubbard model in degenerate perturbation theory in the limit $U / t \rightarrow \infty$ [82] and is considered a minimal model for high-temperature superconductors [83].

\footnotetext{
${ }^{1}$ Standard notation for the operators is used, i.e., $c_{i, \sigma}^{(\dagger)}$ represents a fermionic annihilation (creation) operator, $b_{i}^{(\dagger)}$ the corresponding bosonic one, and $n_{i, \sigma}=c_{i, \sigma}^{\dagger} c_{i, \sigma}$ or $n_{i}=b_{i}^{\dagger} b_{i}$ the densities in the fermionic or bosonic case, respectively. In the case of the $\mathrm{t}$-J-model, the operators $f_{i, \sigma}^{(\dagger)}$ are fermionic ones, but act on a restricted Hilbert space in which double occupancies are forbidden.

${ }^{2}$ The possibility to exploit quantum speed up was actually first envisaged by Y.I. Manin [78] in 1980; it is unclear to me whether Feynman was aware of this. In any case, he seems to be the first one to promote these ideas in the public in the western hemisphere and pursue them.
} 
The fermionic and spin systems considered so far have a SU(2) symmetry and are invariant under the corresponding transformations (e.g., rotation in spin space). From the theoretical side, it is tempting to enhance this symmetry from $\mathrm{SU}(2)$ to $\mathrm{SU}(\mathrm{N})$. This has attracted considerable theoretical attention in recent years, see, e.g., [84-109] and my own contribution [SRM22]. There is a long history of studies of $\mathrm{SU}(N)$ spin systems [84, 110,111] since they become analytically tractable in the large- $N$ limit, and rich phase diagrams have been identified, see, e.g., [110, 112-124], realizing, amongst others, antiferromagnetic phases and valence-bond solids. In 1D, the aforementioned spin-nematic phases have been predicted $[63,64,125,126]$ as well as generalizations of the so-called AKLT state [111, 122, 127-134], which is an archetypical example for topological phases which are briefly revisited in Sec. 4.3. However, as no exact $\mathrm{SU}(N)$ models have been identified in nature, these efforts were broadly considered a theoretical playground.

Recently, however, it has been proposed that systems with such a high symmetry (up to $N=10$ ) can be realized in quantum simulators with ultracold alkaline earth atoms [66]. More specifically, these experiments can realize $\mathrm{SU}(\mathrm{N})$ symmetric generalizations of fermionic Hubbard models

$$
\mathcal{H}_{\mathrm{Hubbard}}^{\mathrm{SU}(\mathrm{N})}=-t \sum_{\substack{\langle i, j\rangle \\ \alpha=1}}^{\alpha=N}\left(f_{\alpha, i}^{\dagger} f_{\alpha, j}+\text { h.c. }\right)+\frac{U}{2} \sum_{i, \alpha \neq \alpha^{\prime}} f_{\alpha, i}^{\dagger} f_{\alpha^{\prime}, i}^{\dagger} f_{\alpha^{\prime}, i} f_{\alpha, i} .
$$

Here, $f_{\alpha, i}^{(\dagger)}$ is a fermionic annihilation (creation) operator for a particle with flavor $\alpha$ on lattice site $i$. Similar to the $\mathrm{SU}(2)$ case, in the limit $U / t \rightarrow \infty$ an effective $\mathrm{SU}(\mathrm{N})$ symmetric Heisenberg model can be derived

$$
\mathcal{H}_{\text {Heisenberg }}^{\mathrm{SU}(\mathrm{N})}=\frac{2 t^{2}}{U} \sum_{\substack{\langle i, j\rangle \\ \alpha, \beta}} S_{\alpha}^{\beta}(i) S_{\beta}^{\alpha}(j)
$$

with the spin operators $S_{\alpha}^{\beta}(i)=f_{\alpha, i}^{\dagger} f_{\beta, i}$. Having this and the specific experimental implementation in mind, exotic new phases have been predicted. An example which has intrigued researchers is the possibility to realize chiral spin liquids $[135,136]$ in such systems [132]. These are spin liquids with certain topological properties which can be of relevance for the realization of topological quantum computers [67, 137].

These findings underline the recent focus of research on the uncovering of new and unconventional behavior in microscopic models and their possible experimental detection. For quasi-1D systems (i.e., chains and ladder geometries), this can be done efficiently using the density matrix renormalization group 
method (DMRG) [39, 40], which is explained in detail in various review articles, e.g., Refs. [138-142] and my own contribution [SRM37]. This method has been applied very successfully for the investigation of phase diagrams and of quantum critical behavior of a multitude of (quasi-)1D systems (see the website [143] for a collection of the publications relying on this method). However, for 2D systems, the area law of entanglement growth $[144,145]$ has inhibited an efficient treatment of the microscopic models of interest. On the other hand, from this point of view it is clear that product states, like a ferromagnetic ground state, are not subject to these difficulties. The question arises where the limitations of this approach lie in practice, and to find means to overcome them, despite the restrictions imposed by the area law. My recent contributions [SRM11, SRM16] (attached in the second part of the thesis) discuss how a proper combination of analytical tools and the DMRG leads to successful investigations of frustrated quasi-2D geometries (see also [69-71, 146, 147]). In particular, as demonstrated in Ref. [146], the accuracy when computing local observables is higher than previously expected, shedding new light onto the possibilities to treat $2 \mathrm{D}$ systems with the standard DMRG. The state of the art for approaching 2D systems using the DMRG is summarized in the Review Article by E. M. Stoudenmire and S. R. White, Ref. [148]; in order to speed up the calculations, it is helpful to parallelize the approach by dividing a single run over several regions in real space as discussed in Ref. [149].

The natural next steps are both, the careful investigation of the crossover from $1 \mathrm{D}$ to $2 \mathrm{D}$ systems using the standard DMRG, and, in parallel, to investigate for new methods which are less subject to the limitations imposed by the area law. Using the standard DMRG, the challenge is to formulate the problem in such a way that the entanglement growth is minimized. This can be done, e.g., by applying appropriate 'pinning fields' at the boundaries and by choosing optimal lattice topologies, as shown in Ref. [146], or by reordering the lattice sites, as discussed in the context of a quantum chemistry approach $[150,151]$. Due to the results of my contributions [SRM11, SRM16] and of $[69-71,146]$, I expect that a fruitful combination of analytical tools and the numerical approaches will lead to interesting new insights into the behavior of two-dimensional quantum many body systems.

Next, I will review some of the most interesting developments in recent years, and discuss the potentiality to realize and further develop such methods in the future. 


\section{Chapter 2}

\section{Matrix Product States and Further Developments of the DMRG}

The density matrix renormalization group (DMRG) method is an approach in which the system is treated in a truncated Hilbert space with size much smaller than the dimension of the actual one. The basis states are selected so that the entanglement in the system is captured in an optimal way. This entanglement aspect of the DMRG method [139] has given an incentive to the development of new methods extending the standard DMRG in the context of quantum information theory. The most important aspect is that the DMRG is a particular realization of a class of algorithms in which a matrix product state wave function (MPS) $[152,153]$ is optimized in a variational way in the course of the iterations $[138,154-156]$. This insight lead immediately to the formulation of algorithms improving on the complexity when treating systems with periodic boundary conditions $[157,158]$. Generalizations of this ansatz to 2D systems are achieved by introducing Projected Entangled Pair States (PEPS) methods [155, 156, 159-162] and tensor-network algorithms like the multi-scale renormalization group ansatz (MERA) [156, 163-166]. It is possible to formulate variants in which the algorithms work directly in the thermodynamic limit exploiting the translational symmetry of the systems and which then are referred to as 'infinite PEPS' or 'infinite MERA' (iPEPS [161] or iMERA [165]), respectively.

At the present stage, optimal implementations as well as further development of these algorithms are a current topic of investigations (see, e.g., [167]). Recently, careful studies have been performed, and (i)PEPS methods 
have been successfully applied, e.g., for treating quantum criticality [168], 2D t-J-models, Eq. (1.7), [169-171], the $S=1 / 2$ Heisenberg antiferromagnet Eq. (1.1) on the 2D Shastry-Sutherland lattice depicted in Fig. 1.1(b) [172, 173] and [SRM3], and systems with SU(N) symmetries [174-176]. The MERA has, e.g., been applied to investigate the ground-state of the $S=1 / 2$ Heisenberg model (1.1) on the kagome geometry [Fig. 1.1(c)], Ref. [177]. Note, however, that the variational energies obtained by Quantum-MonteCarlo methods are lower than the ones obtained by PEPS in the study of Ref. [169], and the energies obtained by the standard DMRG extrapolated to 2D presented in Refs. [69-71] are lower than the ones found by MERA in Ref. [177] (for a detailed discussion for how to approach 2D systems using the DMRG, see the aforementioned review article [148]). It is therefore essential to find ways to improve these approaches by optimizing the implementations and by further developing the methods. A promising path is to combine these MPS-based approaches for variational wave functions with other methods, e.g., stochastic sampling of the configuration space. Very interesting in this context is the introduction of the Minimally Entangled Typical Thermal state algorithm (METTS [178, 179], see also the viewpoint [180]), which treats systems at finite temperatures. In addition, developments combining Quantum Monte Carlo (QMC) methods and MPS might be interesting [181, 182]. Due to their significance, let us quickly review the main features of MPS and PEPS approaches.

\subsection{Matrix product states (MPS) and matrix product operators (MPO)}

In principle, matrix product states provide an exact formulation for generic wave functions. However, the big advantage is that they open in a rather natural way a path for optimizing approximate representations of wave functions. Here, I follow the excellent review [138]. Generically, a many-body wave function on a lattice with $N$ sites can be written as

$$
|\psi\rangle=\sum_{\sigma_{1}, \ldots, \sigma_{N}} c_{\sigma_{1}, \ldots, \sigma_{N}}\left|\sigma_{1} \ldots \sigma_{N}\right\rangle
$$

$\left|\sigma_{i}\right\rangle$ represents a basis state on site $i$, e.g., for a $S=1 / 2$ system $\left|\sigma_{i}\right\rangle=|\uparrow\rangle_{i}$ or $|\downarrow\rangle_{i}$. In general, the site basis has $d$ states (for $S=1 / 2$, hence, $d=2$ ), and the dimension of the Hilbert space of the total system is $d^{N}$. In a matrix product state representation, Eq. (2.1) is rewritten in terms of products of 
matrices, where each of these matrices acts locally:

$$
|\psi\rangle=\sum_{\sigma_{1}, \ldots, \sigma_{N}} \mathbf{A}^{\sigma_{1}} \mathbf{A}^{\sigma_{2}} \ldots \mathbf{A}^{\sigma_{N-1}} \mathbf{A}^{\sigma_{N}}\left|\sigma_{1} \ldots \sigma_{N}\right\rangle
$$

This is achieved by rewriting the coefficients $c_{\sigma_{1}, \ldots, \sigma_{N}}$ as further explained below. The index $\sigma_{i}$ indicates a matrix for each of the states of the site basis. Therefore, we are dealing with $d \cdot N$ matrices, a number which is much smaller than the number of coefficients $c_{\sigma_{1}, \ldots, \sigma_{N}}$ which is the dimension of the system's Hilbert space $d^{N}$. Since, so far, we have only rewritten the coefficients $c_{\sigma_{1}, \ldots, \sigma_{N}}$, not much more is gained. However, as I will discuss below, this ansatz helps in obtaining $|\psi\rangle$ in two ways:

1. Instead of directly computing all of the $d^{N}$ coefficients $c_{\sigma_{1}, \ldots, \sigma_{N}}$ (e.g., by diagonalizing the system's Hamiltonian), one can formulate algorithms which iteratively 'optimize' each of the $d \cdot N$ matrices $\mathbf{A}_{i}^{\sigma}$; i.e., it should be possible to find algorithms for computing $|\psi\rangle$ which do not need an effort exponential, but linear in $N$.

2. It is possible to truncate the dimensions of each of the matrices $\mathbf{A}_{i}^{\sigma}$, which will lead to a substantial speedup and efficiency typical for these type of algorithms, while keeping the error of the approximation small.

The key element for doing so is the singular value decomposition (SVD), according to which any rectangular matrix $\mathbf{M}$ of dimensions $N_{A} \times N_{B}$ can be rewritten as

$$
\mathbf{M}=\mathbf{U S V}^{\dagger}
$$

with $\mathbf{U}$ having orthonormal columns and dimensions $N_{A} \times \min \left(N_{A}, N_{B}\right), \mathbf{S}$ a diagonal matrix of dimension $\min \left(N_{A}, N_{B}\right) \times \min \left(N_{A}, N_{B}\right)$ and $\mathbf{V}^{\dagger}$ a matrix with orthonormal rows and dimensions $\min \left(N_{A}, N_{B}\right) \times N_{B}$. The entries of $\mathbf{S}$ are non-negative and are referred to as singular values, and the number $r$ of non-zero singular values determines the rank of $\mathbf{M}$. Note that the orthonormality of the column- and row-vectors of $\mathbf{U}$ and $\mathbf{V}^{\dagger}$, respectively, allow us to use these as basis vectors. When ordering the singular values in descending order, we can imagine them to reflect the weight or importance of the corresponding columns or rows of $\mathbf{U}$ and $\mathbf{V}^{\dagger}$, respectively. It is natural to ask, if this can be used to introduce a controlled cutoff in the number of basis states used, and to estimate the error introduced by the cutoff. A nice way to see that this is possible is by considering the Schmidt decomposition of the wave function of a system which is divided into two parts $A$ and $B$. With $D$ the 
dimension of the smaller of the Hilbert spaces of $A$ and $B$, we obtain

$$
|\psi\rangle=\sum_{i=1}^{D} s_{i}|A\rangle_{i}|B\rangle_{i} \approx \sum_{i=1}^{m<D} s_{i}|A\rangle_{i}|B\rangle_{i},
$$

with states $|A\rangle$ and $|B\rangle$ living on either part $A$ or $B$, respectively. Several remarks are in order. Eq. (2.4) can be seen as the SVD of the corresponding matrix representation of the wave function: the vector containing the coefficients $c_{\sigma_{1}, \ldots, \sigma_{N}}$ in Eq. (2.1) can be recast to a matrix $\psi_{\left(\sigma_{1}, \ldots, \sigma_{\ell}\right),\left(\sigma_{\ell+1}, \ldots, \sigma_{N}\right)}$ where the sites $1, \ldots \ell$ belong to part $A$ of the system and sites $\ell+1, \ldots, N$ to part $\mathrm{B}$. The SVD of this matrix leads to a decomposition where the states $|A\rangle$ and $|B\rangle$ are represented by the column and row vectors of the corresponding $\mathbf{U}$ and $\mathbf{V}^{\dagger}$ matrices of Eq. (2.3), and the coefficients $s_{i}$ in Eq. (2.4) are the singular values. Furthermore, direct computation of $\operatorname{Tr}_{A / B}|\psi\rangle\langle\psi|$ reveals that the matrices $\mathbf{U}$ and $\mathbf{V}^{\dagger}$ diagonalize the reduced density matrices of system part $A$ and $B$, respectively, so that the states $|A\rangle$ and $|B\rangle$ are the eigenstates of the reduced density matrices, with eigenvalues $s_{i}^{2}$. At this point, we can give a probabilistic interpretation to the Schmidt decomposition Eq. (2.4): the singular values are, indeed, the probability of finding subsystem $A$ or $B$ in the corresponding eigenstate of the reduced density matrix. Therefore, neglecting small values of $s_{i}$ amounts to neglecting less important eigenstates of the reduced density matrices. Hence, introducing the cutoff at $m<D$ states as in the right hand side of Eq. (2.4) is a controlled approximation, and a measure for the error

$$
\varepsilon=\sum_{m+1}^{D} s_{i}^{2}
$$

can be introduced. $\varepsilon$ is called the discarded weight, and in a DMRG calculation the goal is to keep $\varepsilon$ as small as possible, typically being of the order of $10^{-9}$ or smaller. Note that all that has been discussed so far is independent of details of the system like connectivity or spatial dimension. However, as discussed further below, this representation gives the most useful approximations for one-dimensional systems.

The key aspect of the DMRG and of related MPS algorithms is to use these observations to write down the wave function of the system in the form (2.2) and at the same time to introduce a cutoff so that the sizes of the matrices $\mathbf{A}_{\sigma_{i}}$ are small enough to be treated with a reasonable amount of computational resources, while the error is controlled via the discarded weight. In order to see how an arbitrary state can be recast into the MPS form, let us start with a bipartition in which $A$ consists of a single site located at the left end. Let 
us assume that all the lattice sites have the same properties and that the local basis is of dimension $d$. This bipartition hence results in a matrix

$$
\psi_{\left(\sigma_{1}\right),\left(\sigma_{2}, \ldots, \sigma_{N}\right)}=c_{\sigma_{1}, \ldots, \sigma_{N}}
$$

of dimension $d, d^{N-1}$. An SVD results in

$c_{\sigma_{1}, \ldots, \sigma_{N}}=\psi_{\left(\sigma_{1}\right),\left(\sigma_{2}, \ldots, \sigma_{N}\right)}=\sum_{a_{1}}^{r_{1}} U_{\sigma_{1}, a_{1}} s_{a_{1}}\left(V^{\dagger}\right)_{a_{1},\left(\sigma_{2}, \ldots, \sigma_{N}\right)} \equiv \sum_{a_{1}}^{r_{1}} A_{a_{1}}^{\sigma_{1}} \psi_{\left(a_{1} \sigma_{2}\right),\left(\sigma_{3}, \ldots, \sigma_{N}\right)}$,

with rank $r_{1} \leq d$. On the right hand side we have recast the matrix $\mathbf{U}$ of the SVD to a collection of $d$ row vectors $A^{\sigma_{1}}$ with entries $A_{a_{1}}^{\sigma_{1}}=U_{\sigma_{1}, a_{1}}$, and the product of the singular values $s_{a_{1}}$ and the $\mathbf{V}^{\dagger}$ matrix to a new matrix $\psi_{\left(a_{1} \sigma_{2}\right),\left(\sigma_{3}, \ldots, \sigma_{N}\right)}$ of dimension $r_{1} d, d^{N-2}$. Performing a SVD on this new matrix $\psi$ yields in a similar manner

$$
c_{\sigma_{1}, \ldots, \sigma_{N}}=\sum_{a_{1}}^{r_{1}} \sum_{a_{2}}^{r_{2}} A_{a_{1}}^{\sigma_{1}} A_{a_{1}, a_{2}}^{\sigma_{2}} \psi_{\left(a_{2} \sigma_{3}\right),\left(\sigma_{4}, \ldots, \sigma_{N}\right)},
$$

with the matrix $\psi_{\left(a_{2} \sigma_{3}\right),\left(\sigma_{4}, \ldots, \sigma_{N}\right)}$ of dimension $r_{2} d, d^{N-3}$ and the rank $r_{2} \leq$ $r_{1} d \leq d^{2}$. However, instead of vectors as in the step before, we obtained a set of $d$ matrices $\mathbf{A}^{\sigma_{2}}$ each of dimension $r_{1}, r_{2}$ with entries $A_{a_{1}, a_{2}}^{\sigma_{2}}=U_{\left(a_{1} \sigma_{2}\right), a_{2}}$. Repeating this scheme, we can rewrite the wave function as

$$
\begin{aligned}
|\psi\rangle & =\sum_{\sigma_{1}, \ldots, \sigma_{N}} \sum_{a_{1}, \ldots, a_{N-1}} A_{a_{1}}^{\sigma_{1}} A_{a_{1}, a_{2}}^{\sigma_{2}} \cdots A_{a_{N-2}, a_{N-1}}^{\sigma_{N-1}} A_{a_{N-1}}^{\sigma_{N}}\left|\sigma_{1} \ldots \sigma_{N}\right\rangle \\
& =\sum_{\sigma_{1}, \ldots, \sigma_{N}} \mathbf{A}^{\sigma_{1}} \mathbf{A}^{\sigma_{2}} \ldots \mathbf{A}^{\sigma_{N-1}} \mathbf{A}^{\sigma_{N}}\left|\sigma_{1} \ldots \sigma_{N}\right\rangle
\end{aligned}
$$

which is the form (2.2). Note that in the derivation we made so far, $\mathbf{A}^{\sigma_{1}}$ and $\mathbf{A}^{\sigma_{N}}$ are actually vectors. This is because we distinguished sites of the lattice as being the 'first' and the 'last' site of the lattice, and is the reason why the product of matrices results in a scalar. This is fine if we consider a lattice with open boundary conditions. However, for a translationally invariant system there is obviously no reason why they should be treated differently from the matrices in the bulk region of the system. It is, therefore, more accurate to replace the vectors in Eq. (2.7) by matrices. Since the first and the last site are now connected to each other, the MPS is consistent with matrix multiplications on all bonds by taking the trace, so that the MPS wave function now takes on the form

$$
|\psi\rangle=\sum_{\sigma_{1}, \ldots, \sigma_{N}} \operatorname{Tr}\left(\mathbf{A}^{\sigma_{1}} \mathbf{A}^{\sigma_{2}} \cdots \mathbf{A}^{\sigma_{N-1}} \mathbf{A}^{\sigma_{N}}\right)\left|\sigma_{1} \ldots \sigma_{N}\right\rangle .
$$


Since translational invariance is assumed, the dimension of all the matrices $\mathbf{A}^{\sigma_{i}}$ can be chosen to be the same. This was one of the first insights that came from a quantum information approach to the established DMRG method. By carefully writing down the MPS form of the wave function and analyzing the properties of the algorithm, it became clear how to formulate further variants and extensions of the method, e.g., for treating translationally invariant systems directly in the thermodynamic limit, or to generalize the form (2.2) to better suit $2 \mathrm{D}$ systems.

Using the ansatz (2.2) it is possible to search for eigenstates (typically the ground state) of the system and also to compute the time evolution when going out-of-equilibrium. For the sake of brevity, let us briefly sketch the basic ideas for the ground state algorithms, and in the next section some basic properties for time evolution algorithms, and refer to the review [138] for details of the algorithms. The goal is to formulate iterative algorithms which optimize (or in the case of computing the time evolution) update subsequently the matrices $\mathbf{A}^{\sigma_{i}}$. E.g., for the ground state search, one has to find the MPS which minimizes

$$
E_{0}=\frac{\langle\psi|\hat{H}| \psi\rangle}{\langle\psi \mid \psi\rangle} .
$$

This can either be done by computing an imaginary time evolution on some random initial MPS, or by a variational ground state search, which usually is more efficient than the imaginary time evolution. The problem can then be formulated as searching for the extremum of

$$
\langle\psi|\hat{H}| \psi\rangle-\lambda\langle\psi \mid \psi\rangle
$$

with $\lambda$ the Lagrangian multiplier which upon solution will be the ground state energy. By subsequently addressing each of the matrices of the MPS, one obtains in every optimization step a generalized eigenvalue problem whose solution gives the update of the matrix and the lowest lying eigenvalue being the estimate for the ground state energy in this step. This is done until convergence in the energy is obtained, or until the variance of the Hamiltonian $\left\langle\hat{H}^{2}\right\rangle-\langle\hat{H}\rangle^{2}$ is close to zero. In the course of the procedure, one can start first with small dimensions of the matrices and then in later iterations subsequently increase while monitoring the discarded weight. This allows one to control the calculation, for further details, see [138].

Note that, up to now, all that was said is exact and independent of the dimensionality or connectivity of the lattice. However, it is found that the DMRG 
and its MPS variants work best in 1D systems. To elucidate the reason for this behavior, we have to go deeper into quantum information properties and revisit the Schmidt decomposition Eq. (2.4) and the truncation of the basis mentioned there. Often, even for large 1D systems with hundreds or sometimes even thousands of lattice sites, the order of only $m \sim 1000$ basis states need to be kept for obtaining a discarded weight $\varepsilon \sim 10^{-9}$. This is an impressive reduction of the needed computational efforts and is the main reason for the success of the DMRG. In order to be able to work with such small values of $m$, however, the singular values in the Schmidt decomposition need to be 'well behaved', i.e., many of the singular values should have values close to zero or of the order of machine precision (typically $\sim 10^{-16}$ ). The best case scenario is that only a hand full of singular values are substantially larger than zero. It turns out $[139,140]$ that for gapped 1D strongly correlated systems the singular values decay exponentially, so that indeed a small value of $m$ can be used.

The behavior of the singular values is intimately related to the entanglement present in the system. For a pure state, the von Neumann (or entanglement) entropy is a good measure for quantifying the entanglement present in the system [183]. For a bipartition at site $\ell$ it is given by

$$
S_{\ell}=-\operatorname{Tr}_{A / B} \varrho_{\ell} \ln \varrho_{\ell}=-\sum_{i} s_{i}^{2} \ln s_{i}^{2} .
$$

For a pure product state, one of the singular values $s_{1}=1$, and the others are exactly zero, leading to $S_{\ell}=0$. Obviously, the DMRG/MPS algorithms are expected to work best in this case. On the other hand, in a maximally entangled state, all the singular values have (per definitionem) the same value with $s_{i}^{2}=1 / d^{\ell}$ for a bipartition where the smaller subsystem has $\ell$ sites. In this case, $S_{\ell}=\ell \ln d$ becomes maximal, and any cutoff to $m$ states leads to a substantial error since all basis states in the Schmidt decomposition have the same importance. Hence, the larger the entanglement in the system, the larger the number of states $m$ needed to reach a certain accuracy. The best case for the DMRG/MPS algorithms is therefore a system which is not too strongly entangled. In [37] it is shown that this is the case in gapped 1D systems since here $S_{\ell}=$ const. for $\ell$ larger than the correlation length. The representation of $|\psi\rangle$ can therefore be exact with a small value of $m$ since in the derivation of Eq. (2.2) the rank of the matrices $\mathbf{A}^{\sigma_{i}}$ played a role, which can be small if many singular values are zero. Gapless 1D systems are already more difficult to treat since here according to Eq. (1.3) the entanglement entropy grows logarithmically with $\ell$. Hence, the above statement that the representation of $|\psi\rangle$ can be exact with a small value of 
$m$ does not hold any more, but a good approximation with small discarded weight can be obtained for large enough values of $m .^{1}$ The observation of a constant entanglement entropy for gapped 1D systems is a manifestation of the area law of entanglement, according to which the entanglement present in the system is $\sim D^{-1}$, i.e., to the surface of the subsystem ${ }^{2}[145,185]$. In higher dimensions, the situation is therefore worse and the question arises for a better representation of the wave function which takes into account the area law correctly. In the next section, I briefly discuss the PEPS ansatz which is a straight-forward generalization of MPS to higher dimensions taking into account the entanglement area law.

At the end of this section, let us briefly discuss two aspects which have been found to be useful for the treatment of translationally invariant systems and for systems with long-range interactions. In the presence of translational invariance, one can revisit the MPS wave function for systems with periodic boundary conditions (2.8), in which we had chosen all the matrices $\mathbf{A}^{\sigma_{i}}$ to have the same dimensions. Going one step further, one realizes that in the presence of translational invariance, all of these matrices are identical. Hence, it is possible to formulate algorithms in which only a few matrices (sufficient to describe the unit cell of possibly emerging patterns in the system) are optimized and by periodic repetition of this small number of matrices one can directly compute expectation values of observables in the thermodynamic limit.

In order to compute observables, and also to perform the optimization procedure, one needs to apply operators to MPS. This is done by formulating them in terms of matrix product operators (MPO). The basic idea is to recast the operator into an expression in terms of local matrices, similar to the MPS idea. For a generic operator, this leads to the form

$$
\hat{O}=\sum_{\substack{\sigma_{1}, \ldots, \sigma_{N} \\ \sigma_{1}^{\prime}, \ldots, \sigma_{N}^{\prime}}} \mathbf{W}^{\sigma_{1}, \sigma_{1}^{\prime}} \mathbf{W}^{\sigma_{2}, \sigma_{2}^{\prime}} \ldots \mathbf{W}^{\sigma_{N-1}, \sigma_{N-1}^{\prime}} \mathbf{W}^{\sigma_{N}, \sigma_{N}^{\prime}}\left|\sigma_{1}, \ldots \sigma_{N}\right\rangle\left\langle\sigma_{1}^{\prime}, \ldots, \sigma_{N}^{\prime}\right|
$$

For periodic boundary conditions, the same extension as in Eq. (2.8) is obtained. To discuss the technical details when applying the MPOs to MPS

\footnotetext{
${ }^{1}$ When treating finite systems, one always has a finite size gap, so that in practice one is always dealing with gapped systems which are more beneficial to the truncation. However, close to a gapless point (e.g., a phase transition) or in a critical phase, the value of $m$ can grow substantially also in 1D.

${ }^{2}$ Note that the entanglement can grow even faster with an additional logarithmic factor, e.g., in critical 1D systems discussed previously, see Eq. (1.3), and in critical 2D systems as discussed in [145, 184].
} 
would lead to far at this point, and I refer again to the review [138]. Let me illustrate the power of the MPO formulation on a concrete example. In my contribution [SRM5] (attached in the second part of the thesis), we followed $[186,187]$ and use this formulation for efficiently treating systems with dipolar long-range interactions. As discussed in more detail there and in the supplementary material of [SRM5], this leads to a very accurate representation of the Hamiltonian of the long-range systems, resulting in a substantial speed up of the calculations. Despite the interactions going over long distances, this approach allowed us to treat systems with as many lattice sites as in a comparable system with short-ranged interactions.

\subsection{Projected Entangled Pair States (PEPS)}

As discussed above, one major problem when dealing with systems in spatial dimensions $D>1$ is the area law of entanglement which makes it necessary to keep a large number of basis states in order to obtain a sufficiently small discarded weight. One approach to overcome this problem is therefore to generalize the MPS state to an ansatz which can better capture the entanglement in the higher dimensional systems. This is realized by the so-called 'PEPS' ansatz (projected entangled pair states) [155, 159]. In the following I summarize briefly the main features of this ansatz following the presentation in $[155]$.

The basic observation is that in an MPS ansatz, the number of degrees of freedom captured by the approach is bounded by the introduced cutoff $m$. However, due to the area law, the number of needed degrees of freedom needed to describe the behavior of the subsystem has to scale as the boundary of the subsystem, so that the states needed to keep the discarded weight small grow exponentially with the size of the boundary. The goal is, hence, to formulate an ansatz which captures this exponential growth of the number of degrees of freedom.

The PEPS are, as the MPS, a generic ansatz which allow to represent any quantum state. For the sake of simplicity, I will focus on PEPS on a square lattice, generalizations to further geometries and higher dimensions are straight forward. Let us start by representing each site $i$ by four auxiliary systems $a_{i}, b_{i}, c_{i}$ and $d_{i}$. Let us choose them each to be in a maximally entangled state with one of the four neighbors of the site, respectively. The PEPS is obtained by applying an operator $\hat{Q}_{i}$ to map the four auxiliary systems to one physical site with dimension of the site basis $d$. The coefficients of the 
resulting state are formulated as contractions of tensors, which are related to the operator $\hat{Q}_{i}$ on the site via

$$
\left[A_{i}\right]_{l, r, u, d}^{k}=\left\langle k\left|\hat{Q}_{i}\right| l, r, u, d\right\rangle ;
$$

I denote by $|k\rangle$ the $d$ physical states on the site, and introduce four virtual indices $l, r, u, d$ with dimension $m$. Hence, similar to the MPS ansatz in which we introduced local matrices $\mathbf{A}^{\sigma_{i}}$, we have now introduced local tensors attached to site $i$. The PEPS is obtained by contracting the tensors by a scheme which mimics the underlying lattice structure. In our case, the four virtual indices are related to the left, right, upper and lower bonds emanating from the respective neighboring sites. The coefficients of the PEPS are obtained by joining the tensors in such a way that all virtual indices related to the same bonds are contracted. With $\mathcal{F}(\cdot)$ the function which performs this contraction, we can finally formulate the PEPS wave function as

$$
|\psi\rangle=\sum_{k_{1}, \ldots, k_{N}=1}^{d} \mathcal{F}\left(\left[A_{1}\right]^{k_{1}}, \ldots,\left[A_{N}\right]^{k_{N}}\right)\left|k_{1}, \ldots, k_{N}\right\rangle .
$$

Note the resemblance to the MPS Eqs. (2.2) and (2.8). However, the generalization using tensors now allows to generalize this construction to any lattice shape and dimension, and for bond dimensions $m$ large enough, any quantum state can be written as a PEPS. Note that now the entanglement area law is captured: in contrast to the MPS, the number of bonds connecting the subsystem and the rest of the system is now proportional to the surface of the subsystem. Hence, each bond can stay at a smaller bond dimension and still capture effectively the entropy, so that a good approximation of the wave function can be obtained at smaller values of $m$. Indeed, Hastings [188] has shown that every ground state of local quantum spin Hamiltonians can be efficiently represented by a MPS (and hence also a PEPS) also in higher dimensions with bond dimensions $m$ scaling sub-exponentially with the number of spins of interest. Interestingly, he also shows that all thermal states have an efficient representation in terms of MPOs. Therefore, MPS, PEPS and MPOs seem to form the relevant manifold for describing the low-energy behavior of quantum spin systems. At the moment, an important task is to obtain efficient implementations of algorithms for optimizing PEPS wave functions allowing one to treat bond dimensions large enough for exploring interesting behavior in a rather unbiased way similar to the progress obtained by the DMRG for $1 \mathrm{D}$ systems.

To conclude, let us remark that it is possible to formulate the PEPS $|\psi\rangle$ directly in the thermodynamic limit in the presence of translational invariance by keeping as many tensors as needed to capture the unit cell of the 
periodic pattern in the system. This variant, coined iPEPS [161], seems at the moment to lead to the most stable and efficient formulation of algorithms for optimizing PEPS wave functions and has been applied in the context of various systems, see, e.g., [168-176]. Let me illustrate the possibilities of this approach on the example of my own contribution [SRM3] (attached in the second part of the thesis) in which the 2D Shastry-Sutherland lattice in a magnetic field is analyzed using various methods, including the standard DMRG and iPEPS. The results of these three numerical methods agree very well. However, since the iPEPS are working directly in the thermodynamic limit, it is possible to avoid problems with the commensurability of the resulting periodic structures on the system and gain a better control on the behavior of the full 2D system without need for performing a difficult finite size scaling analysis. The prize needed to be paid is that the computations need to be done with a variety of possible unit cells, always leaving the possibility open that even larger unit cells could be a better solution of the system.

\subsection{Exploring out-of-equilibrium systems with the adaptive time-dependent DMRG}

An important breakthrough in the development of numerical methods for systems out-of-equilibrium was achieved in the years 2003-2004. The DMRG can be extended to the adaptive time-dependent DMRG (adaptive t-DMRG) [189-194] which allows for the efficient and accurate treatment of non-equilibrium situations [195]. My own contribution [SRM38] and the time-step targeting approach of Ref. [196] pursue the development of variants of the adaptive t-DMRG; in particular, [SRM38] shows how to combine the adaptive t-DMRG with a Krylov-space representation of the time evolution operator. This allows for a larger flexibility of the method (for further details see below and my $\mathrm{PhD}$ thesis [SRMPhD]), e.g., for treating systems with long-range interactions as in my contributions [SRM2] and [SRM6, SRM10] (attached in the second part of the thesis). It is possible to extend the adaptive t-DMRG to treat finite temperature properties $[197,198]$ by performing an imaginary time evolution on a purification of the thermal density matrix by adding an ancilla space to the system or by directly implementing the thermal density matrix using MPOs [198, 199]. This can be used to compute observables and thermodynamic properties, and also to obtain dynamical spectral functions at zero and finite temperatures [192, 200-203] by comput- 
ing the Fourier-transform of time dependent correlation functions; alternatively, a combination of the finite-temperature Lanczos approach [204, 205] with DMRG has been proposed which requires stochastic sampling [206]. In [207] it is demonstrated that performing the real-time evolution backward in time on the ancilla space leads to a smaller growth of entanglement so that longer times can be reached. Alternative approaches work directly in frequency space at zero temperatures, e.g., the so-called dynamical DMRG (DDMRG) [208-210]. In a recent own contribution it is shown how to formulate a Liouvillian approach for the calculation of dynamical spectral functions at finite temperatures directly in frequency space with a high resolution [SRM1] which also gives a natural framework to the backward time evolution on the ancilla space of [207]. Further developments of the adaptive t-DMRG treat dissipative systems either by exploiting the properties of the superoperator algorithm [197, 198], by using a formulation of the t-DMRG in the Heisenberg-picture [211, 212], or by combining the t-DMRG with quantumtrajectory-approaches [213] (see also the viewpoint [214]).

Since the adaptive t-DMRG has become an important tool for treating nonequilibrium situations of low-dimensional quantum many body systems, I want to quickly review it with its most important variants, following the presentation in [138]. In most of the cases one uses one of two variants for computing the time evolution within MPS/DMRG approaches [SRM38]. The first one relies on the Suzuki-Trotter decomposition of the time evolution operator, e.g., to first order

$$
e^{-i \hat{H} \Delta t}=e^{-i \hat{h}_{1} \Delta t} e^{-i \hat{h}_{2} \Delta t} \cdots e^{-i \hat{h}_{L-2} \Delta t} e^{-i \hat{h}_{L-1} \Delta t}+\mathcal{O}\left([\Delta t]^{2}\right)
$$

here we assumed that only nearest neighbor interaction terms come into play and that the Hamiltonian of a 1D system with $L$ lattice sites can be written as $\hat{H}=\sum_{i=1}^{L-1} \hat{h}_{i}$ with the local operators $\hat{h}_{i}$ acting on the bonds. For nearest neighbor interactions, the time evolution operators on the odd bonds and on the even bonds, respectively, mutually commute, so that we can group them to $\hat{U}_{\text {odd }}=e^{-i \hat{H}_{\text {odd }} \Delta t}$ and $\hat{U}_{\text {even }}=e^{-i \hat{H}_{\text {even }} \Delta t}$, respectively. The time evolution on either the odd or the even bonds can now be computed in a single step by formulating the MPO for either $\hat{U}_{\text {odd }}$ or $\hat{U}_{\text {even }}$ and applying first the one to the MPS representing $|\psi(t)\rangle$, and then the other ${ }^{3}$. The algorithm can then be summarized as follows:

\footnotetext{
${ }^{3}$ In the standard DMRG formulation, one can perform half a sweep to apply $\hat{U}_{\text {odd }}$ and afterwards another half sweep back throughout the system to apply $\hat{U}_{\text {even }}$.
} 
1. Apply the MPO for $\hat{U}_{\text {odd }}$ to $|\psi(t)\rangle$.

2. Apply the MPO for $\hat{U}_{\text {even }}$ to the result of the previous step. The result is $|\psi(t+\Delta t)\rangle$.

3. Compress $|\psi(t+\Delta t)\rangle$ from dimensions $d^{2} D$ to $D$ and monitor the error.

4. Compute the observables at time steps of interest by evaluating $\langle\hat{O}(t)\rangle=$ $\langle\psi(t)|\hat{O}| \psi(t)\rangle$.

5. Continue with step 1 . until the final time $T_{\max }$ of the simulation is reached.

More details can be found in Ref. [138]. Note that two main error sources come into play: 1) the error of the Trotter-Suzuki decomposition; 2) the error from the compression (or basis truncation in the standard DMRG language). While the first can be accommodated with by going to, e.g., higher orders or using smaller time steps, the second one is a more fundamental problem intrinsic to interacting quantum systems. In the course of the time evolution, the entanglement typically grows, so that $D$ needs to grow with time; typically, an exponential growth in time is found. Therefore, the times reachable are quite restricted, and typically time scales $\sim 10-100$ in the units of the energy can be reached. In order to reach longer times, it is beneficial to try to start from a state which is close to a product state, so that the entanglement in the initial state is minimized. As shown in my own contribution [SRM17] (attached in the second part of the thesis), for an initially dimerized system in this way times $\sim 200$ in the units of the energy could be reached.

The approach we discussed so far relies on the Trotter-Suzuki decomposition of the time evolution operator and on the fact that the Hamiltonian consists only of nearest-neighbor terms. It is possible to treat systems with longer range interactions by formulating MPOs for more than a single bond, or by using an alternative Krylov-space approach in which the time evolution operator is projected onto a basis of so-called Lanczos vectors. In this approach, the iterative procedure

$$
\begin{aligned}
\left|v_{n+1}\right\rangle=\hat{H}\left|v_{n}\right\rangle-\alpha_{n}\left|v_{n}\right\rangle-\beta_{n}^{2}\left|v_{n-1},\right\rangle \\
\alpha_{n}=\frac{\left\langle v_{n}|\hat{H}| v_{n}\right\rangle}{\left\langle v_{n} \mid v_{n}\right\rangle} ; \quad \beta_{n}^{2}=\frac{\left\langle v_{n} \mid v_{n}\right\rangle}{\left\langle v_{n-1} \mid v_{n-1}\right\rangle}
\end{aligned}
$$

forms a basis of vectors $\left\{\left|v_{n}\right\rangle\right\}$ which can be used to represent the time evolution operator (for more details, see, e.g., my contributions [SRM37, SRM38] 
and references therein.) One can now set $\left|v_{0}\right\rangle \equiv|\psi(t)\rangle$ and compute

$$
|\psi(t+\Delta t)\rangle \approx \mathbf{V}_{n} e^{-i \Delta t \mathbf{T}_{n}} \mathbf{V}_{n}^{\dagger}|\psi(t)\rangle
$$

where $\mathbf{V}_{n}$ is the matrix containing the vectors $\left|v_{n}\right\rangle$ in the columns and $\mathbf{T}_{n}$ the Hamiltonian represented in the basis $\left\{\left|v_{n}\right\rangle\right\}$, which takes a tridiagonal form. In this approximation, the Hamiltonian can be of arbitrary range and this ansatz can be used to compute the time evolution for systems with longrange interactions as, e.g., shown in my contributions [SRM2] and [SRM6, SRM10] (attached in the second part of the thesis).

With this, I close my quick overview on numerical methods in the framework of matrix product state ansatzes. The following chapters discuss examples for interesting behavior uncovered using the DMRG and the adaptive t-DMRG in equilibrium as well as out-of-equilibrium in the context of quantum magnetic materials and of quantum simulators realized on optical lattices. 


\section{Chapter 3}

\section{Quantum Magnetism in Strong Magnetic Fields}

As mentioned in Ch. 1, an interesting class of strongly correlated systems is found in so-called quantum magnetic materials. The interplay of interactions, lattice geometries and external fields opens a vast playground for realizing interesting scenarios with unconventional phases of matter as well as unusual phase transitions.

\subsection{Unconventional Phases and Quantum Crit- ical Behavior}

Inspired by quantum magnetic materials, microscopic models have been formulated, and in addition to their possible realization in the materials, it is interesting per se to investigate their properties and search from the theoretical perspective for new, unconventional behavior. An example for which I obtained the complete phase diagram (see my contribution [SRM25] attached in the second part of the thesis) is the $S=1$ Heisenberg-type chain with additional biquadratic interactions in a magnetic field,

$$
\mathcal{H}=\sum_{i}\left[\cos (\theta) \vec{S}_{i} \cdot \vec{S}_{i+1}+\sin (\theta)\left(\vec{S}_{i} \cdot \vec{S}_{i+1}\right)^{2}\right]-B \sum_{i} S_{i}^{z}
$$

the so called bilinear biquadratic Hamiltonian (BLBQ). Here, the simple Heisenberg model of Eq. (1.1) has been extended by a term which also 


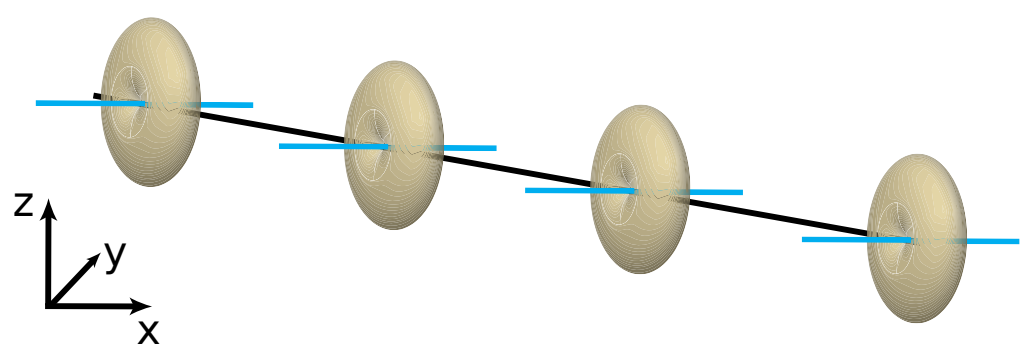

Figure 3.1: Example for an unconventional state realized in low-dimensional spin systems: sketch of a ferroquadrupolar state (taken from my contribution [SRM25], attached in the second part of the thesis; (C) by APS). Note that for this type of ordered state, the $\mathrm{SU}(2)$ symmetry of the system can be spontaneously broken while the local magnetizations remain zero. The emergent order can be visualized as fluctuations around the directors which in this case align parallel to each other.

conserves the $\mathrm{SU}(2)$ symmetry of the system, but acts as a competing interaction. As discussed in [SRM25] the resulting phase diagram is indeed rich. Here, I want to highlight one feature, namely the uncovering of a ferroquadrupolar Luttinger liquid (LL) phase [depicted in Fig. 3.1] realized at finite magnetic fields. Such a phase is one possible realization of spinnematic quasi long range order (QLRO) in $1 \mathrm{D}^{1}$. In higher dimensions, this would correspond to an ordered phase with local order parameter in which the $\mathrm{SU}(2)$ symmetry of the system is spontaneously broken without simultaneously breaking time reversal invariance as in a system with magnetic long range order ${ }^{2}$. In model (3.1), this phase undergoes an unconventional continuous pair-unbinding transition with central charge $c=3 / 2$ to the well-known Luttinger liquid realized in the magnetized Haldane phase [218]. Although at finite magnetizations the value of the central charge is the same as at the integrable point $\theta=-\pi / 4$ at zero field, at which the system is described by a $S U(2)_{2}$ Wess-Zumino-Witten-Novikov theory, the value $c=3 / 2$ in this case is not due to the extended $\mathrm{SU}(2)$ symmetry. Instead, my findings indicate

\footnotetext{
${ }^{1}$ At zero temperature, in higher dimensions continuous symmetries can be spontaneously broken and so lead to 'true' long range order. In 1D, however, this is forbidden by the Mermin-Wagner-Hohenberg theorem [215-217].

${ }^{2}$ Strictly speaking, with a finite magnetic field the SU(2) symmetry of the system has already been reduced to $\mathrm{U}(1)$, and a finite magnetization can be present without spontaneously breaking the $\mathrm{SU}(2)$ symmetry. Nevertheless it is interesting to see that the spin nematic correlation functions become dominant, so that this nomenclature for the phase can be used. Details are discussed in [SRM25] attached in the second part of this thesis.
} 
that the transition realizes a combination of a LL with $c=1$ and an Ising transition which adds a contribution of $c=1 / 2$.

Other systems showing interesting quantum critical behavior in magnetic fields are $S \geq 1 / 2$ frustrated Heisenberg ladders, in which the geometrical frustration leads to a series of magnetization plateaux (the number of which depending on the value of $S$ ) and phase transitions on the plateaux, see my own contribution [SRM27] attached in the second part of this thesis and references therein. Going beyond these (quasi)-1D geometries, unconventional behavior in magnetic fields is studied in the context of the quantum magnetic material $\mathrm{SrCu}_{2}\left(\mathrm{BO}_{3}\right)_{2}$ which is a very good realization of the Shastry-Sutherland lattice [219] depicted in Fig. 1.1(b) [11, 12]. My own contributions [SRM3, SRM11, SRM16] attached in the second part of this thesis address the $T=0$ phase diagram of this system. In Ref. [SRM11], the phase diagram at finite magnetic fields for a quasi-1D version of the lattice is obtained. Interestingly, an unconventional Mott-insulator which is a Wigner crystal of bound states of triplons is uncovered, realized on a magnetization plateau for intermediate values of the couplings which are not amenable to perturbative approaches. In [19], it is argued that such a bound state of triplons cannot be realized in the Shastry-Sutherland system, so this finding in a quasi-1D version of the model is quite interesting. In the meantime, using iPEPS it has been confirmed that, indeed, it is possible to have such bound states of triplons also in the full 2D system where they realize interesting Mott insulators on magnetization plateaus [172, 173]. [SRM11] is an interesting contribution not only because of the finding of this unconventional Wigner crystal, but also from the methods side. In order to obtain the phase diagram, it was essential to combine an analytical perturbative approach ('perturbative continuous unitary transformations', PCUTs [220]) with the DMRG. The latter encounters difficulties due to subtle boundary effects, convergence issues due to the strongly dimerized nature of the system, and commensurability issues for the states with a unit-cell different from the one of the original system. These are, essentially, the difficulties one will encounter with any variational method when treating finite (quasi-)2D lattices. In this specific case, the convergence difficulties could be overcome by invoking some modifications during the sweeps or by adding additional interactions. For the interpretation of the results, it was crucial to perform a careful analysis of the numerical data based on the analytical results obtained in the perturbative limit, and with this knowledge at hand, the behavior in the intermediate regime could be analyzed, leading to the uncovering of the aforementioned unconventional Mott-insulator state. In the meantime, we have extended the study to treat tube geometries which are broader in the 
transverse direction, see my contribution [SRM16] attached in the second part of the thesis. Again, a combination of the perturbative approach and of the DMRG was very helpful in identifying the physical state and the mechanisms leading to its realization, in particular on some magnetization plateaus which are now seen to be understood in terms of highly delocalized and entangled structures. Interestingly, in this case it was the DMRG guiding the development of effective models: in the simple PCUT effective model, the ground state energy per site at a magnetization plateau at $M=1 / 8$ is slightly higher than the DMRG result, with a difference of only $\sim 10^{-4} \mathrm{~J}$. However, the structure of the associated Mott insulator is completely different from the DMRG result. This spurred the development of more accurate effective models including correlated hopping terms (see [SRM16] for more details). This leads to a lowering of the energy by $\sim 10^{-4} \mathrm{~J}$, and a structure of the magnetization plateau which now is in very good agreement to the DMRG result. The delocalization of triplons due to the correlated hopping then leads to the aforementioned highly entangled structures.

My contribution [SRM3] (attached in the second part of the thesis) aims directly at the study of the 2D system by combining a variety of methods, in particular also the DMRG and iPEPS, and a comparison to experiments in magnetic fields up to $118 \mathrm{~T}$ is provided. From $[172,173]$ the impression arises that the iPEPS is particularly suited for treating such strongly dimerized systems, and, indeed, the standard DMRG results of [SRM3] support the picture obtained from iPEPS: The DMRG calculations in [SRM3] are done with periodic boundary conditions in both spatial directions. Although this is a very difficult situation for the DMRG, in this case the dimerization helps because it leads to a structure closer to a product state of dimers than in other systems and hence to a lower entanglement. Computing the magnetization curve is then feasible with a good accuracy since only ground state energies at the specific values of the magnetization need to be performed (for more details see the supplementary material attached in the second part of the thesis right after [SRM3]). The DMRG approach working on finite systems is hence complementary to the iPEPS approach which is working directly in the thermodynamic limit, but plagued by the uncertainties on the size of the unit cells involved and possible convergence issues due to the complexity of the algorithm. The agreement between the results of these two approaches (and also further methods) in [SRM3] allows us to make theoretical predictions and compare to the experimental findings. The excellent agreement of the theoretical and experimental results (also concerning the sequence of magnetization plateaus up to magnetization $M=1 / 2$ ) leads us to conclude that the ratio of the inter- and intra-dimer couplings $J^{\prime} / J \approx 0.63$, and to 

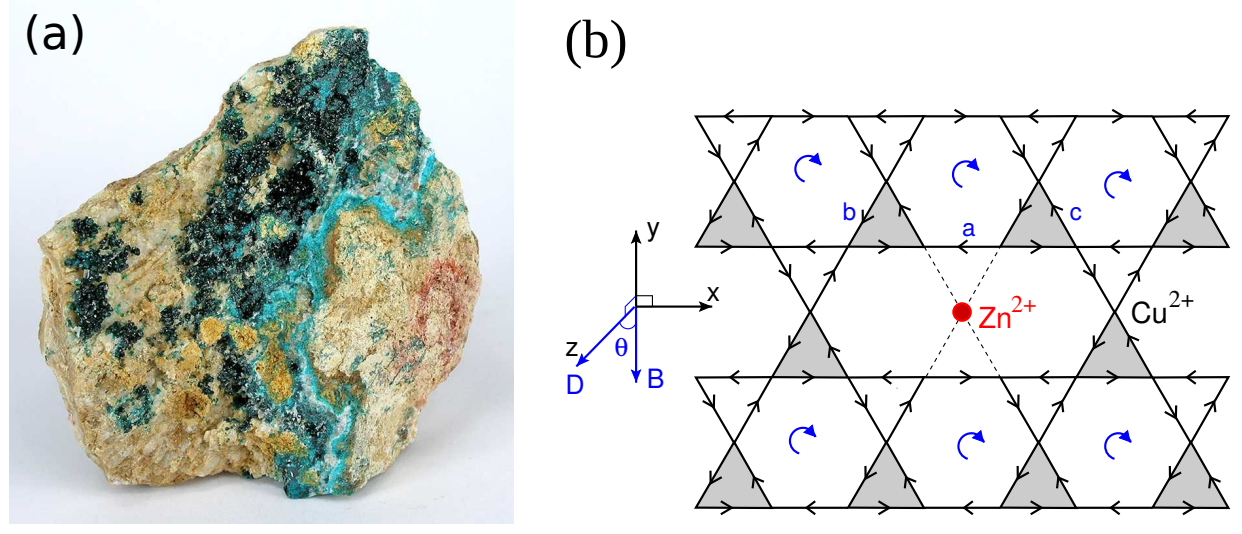

Figure 3.2: (a) The natural mineral Herbertsmithite is the until now best known realization of a Heisenberg $S=1 / 2$ system on the kagome lattice [13]. However, the material contains $\sim 5 \%$ nonmagnetic impurities and anisotropic DM interactions due to spin-orbit coupling (picture from Wikipedia). (b) The lattice with one impurity and the orientation of the DM-vectors used in our study [SRM28] (picture taken from this publication; (C) by APS).

predict that the phases between the plateaux could be supersolids.

\subsection{Effect of Spin-Orbit Coupling at High Mag- netic Fields}

The models mentioned so far are often minimal models. However, in real materials additional effects like spin-orbit coupling are present. In the second part of this thesis, some of my own contributions are attached which focus on a more realistic modeling of such materials. These address $S=1 / 2$ ladder systems [SRM14, SRM30] (see Fig. 1.1(a)), the kagome material Herbertsmithite [SRM28] (Figs. 1.1(c) and 3.2), and the $S=1 / 2$ chain material $\mathrm{Cu}-\mathrm{PM}$ (copper pyrimidine dinitrate [SRM12, SRM1], Fig. 3.3). A common aspect of these materials is the presence of spin-orbit couplings, which in a magnetic field can alter the physics of the system significantly [221], but usually have been neglected in the past due to their smallness. In order to obtain a more realistic description, it is necessary to consider this effect by treating additional anisotropic interactions of the Dzyaloshinskii-Moriya 
(a)

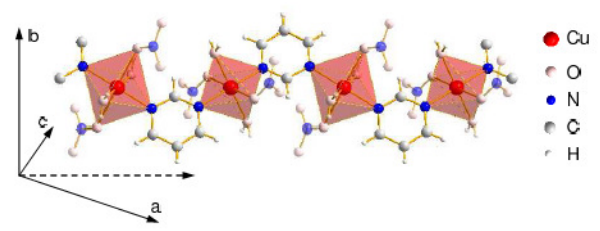

(b)

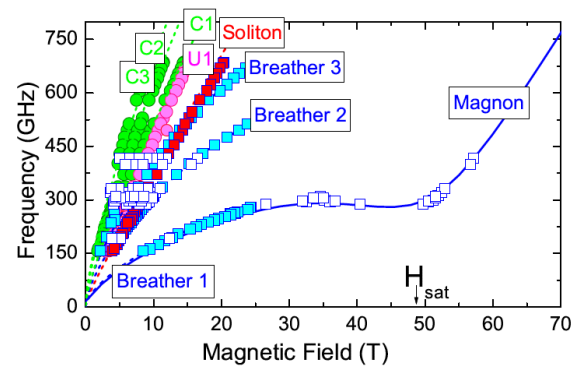

Figure 3.3: (a) Crystal structure of $\mathrm{Cu}-\mathrm{PM}$ (copper pyrimidine dinitrate), a $S=1 / 2$ spin chain material with DM interactions and alternating gtensor. (b) Comparison of ESR spectra (symbols) and DMRG results (solid line) from my own contribution [SRM12] (attached in the second part of the thesis. Both figures are taken from this publication; (c) by APS).

(DM) type [222, 223],

$$
\mathcal{H}_{\mathrm{DM}}=\sum_{\langle i, j\rangle} \vec{D}_{i, j} \cdot\left(\vec{S}_{i} \times \vec{S}_{j}\right)
$$

This term arises in the strong coupling limit of the fermionic Hubbard model (1.5) when taking into account spin orbit coupling $\sim \vec{L} \cdot \vec{S}$. Note that, in contrast to the Heisenberg term (1.1), the DM interaction is antisymmetric upon exchange of the spins and breaks the $\mathrm{SU}(2)$ symmetry. On dimers, this leads to a mixing of the singlet and the triplet sectors and can so lead to new interesting effects.

My contributions cover various aspects of the effects of DM interactions in 1D systems, ladder systems and 2D kagome systems: Contribution [SRM14] (attached in the second part of the thesis) addresses how DM interactions affect torque measurements of the magnetization in experiments. Interestingly, pronounced peaks at the ends of magnetization plateaux can be realized which we propose to use for high precision measurements of the endpoints of magnetization plateaux. My contribution [SRM28] (attached in the second part of the thesis) sheds some light onto NMR experiments on the kagome material Herbertsmithite by addressing a kagome system in the presence of nonmagnetic impurities and DM interactions using exact diagonalizations. Our findings support that the findings of NMR experiments at low temperatures (in particular a remnant line shift indicating the presence of two different magnetizations at the low field strengths used) [224] can be directly explained by the presence of DM interactions. Our results indicate the strength of the 
DM interaction to be of the order of $0.06 \lesssim|\vec{D} / J| \lesssim 0.1$, in agreement with ESR measurements [225] which find $|\vec{D} / J| \sim 0.08$. My contribution [SRM12] (attached in the second part of the thesis) provides a direct comparison of experimental and numerical DMRG data for CU-PM which is a $1 \mathrm{D}$ Heisenberg $S=1 / 2$ chain material with DM interactions [226, 227]. The experiments go up to field strengths $\sim 60 \mathrm{~T}$, identifying unconventional behavior of the gap. This behavior can be understood by an effective sine-gordon model which predicts various soliton- and breather-like excitations [227-231] which, however is predicted to be valid rather at low magnetic fields. The agreement between the DMRG and the experimental results confirms the validity of the underlying microscopic model for this system, i.e., that the description in terms of a Heisenberg chain with additional DM interactions is valid for all values of the magnetic field. The recent contribution [SRM1] takes this model as an example for demonstrating the accuracy of a recently developed Liouville approach to compute dynamical spectral functions at finite temperatures directly in frequency space. It will be interesting to compute ESR line shapes to make predictions for this and further systems for ongoing and future experiments. Note that for the Shastry-Sutherland system $\mathrm{SrCu}_{2}\left(\mathrm{BO}_{3}\right)_{2}$, DM interaction is also of relevance [232-237], but we have neglected it at the present stage and instead have focused on the crossover from quasi-1D to $2 \mathrm{D}$ as described in the previous section.

It remains a challenge to apply numerical methods to further interesting quantum magnetic systems in the future. With increasing experience and optimization of the codes and methods, it is well imaginable that on an intermediate time scale further interesting insights can be obtained in 2D systems, e.g., in the uncovering and characterization of spin liquid phases. The hope is that these approaches are complemented by quantum simulation of the systems in experiments with ultracold gases on optical lattices which I will describe in the next chapter. 


\section{Chapter 4}

\section{Quantum Simulation with Ultracold Atoms and Molecules}

As we have seen in the previous chapters, there is a lot of interesting behavior in correlated systems. Unfortunately, they are often very hard to describe theoretically, even with elaborate numerical tools like the ones presented in Chapter 2. An alternative was envisaged by Y.I. Manin and most prominently by R.P. Feynman at the beginning of the 1980s in quantum computation and for particular problems so-called quantum simulators [7881]. These are experiments aiming at the realization of the same or similar behavior as the system of interest (e.g., a frustrated spin model), however, in an experimental setup which is easier to study than the original one (e.g., some material which is plagued by defects). This is explored in ongoing experiments with ultracold atomic and molecular gases on optical lattices which directly aim at the realization of microscopic models as the ones discussed above in a condensed matter context, see e.g. [1, 2, 238, 239]. The goal is to reach insights into yet open questions, e.g., in the context of hightemperature superconductivity, and to realize novel behavior, as, e.g., new states of matter. Major challenges are the realization of $\mathrm{t}-\mathrm{J}$ and fermionic Hubbard models on optical lattices and to cool them down to temperatures low enough to be able to observe the interesting behavior [239-241]. At this point, I want to highlight two recent developments which are very promising concerning the realization of unconventional states of matter in such quantum simulators: the first development allows for the realization of systems with enhanced $\mathrm{SU}(\mathrm{N})$ symmetry (specifically, the $\mathrm{SU}(\mathrm{N})$ symmetric version of the 
Hubbard model, (1.8)) by cooling down alkaline earth atoms. The second development uses ultracold polar molecules instead of gases of atoms on optical lattices. As further discussed below, this allows for the realization of spin systems (e.g., the Heisenberg model (1.1)) and a generalized version of the t-J-model (1.7). My own contributions to the field can be divided into three groups: i) [SRM22] (attached in the second part of the thesis) and [SRM8] deal with properties of $\mathrm{SU}(\mathrm{N})$ Hubbard like systems. ii) [SRM10, SRM5, SRM6] (attached in the second part of the thesis) and [SRM24] discuss effective models for ultracold polar molecules on optical lattices and how to probe the behavior through non-equilibrium dynamics. iii) [SRM10, SRM6, SRM13, SRM17, SRM18, SRM20, SRM21, SRM23, SRM29] (attached in the second part of the thesis) and [SRM2, SRM15, SRM19, SRM32] and the submitted [SRM39] deal with various aspects of the non-equilibrium dynamics in setups which are of direct relevance for existing quantum simulation experiments or which can be probed in possible future experiments. In this chapter, I will focus on aspects i) and ii) while the next chapter will further discuss the non-equilibrium dynamics.

\subsection{Alkaline Earth Atoms Realization of SU(N) Symmetric Systems}

The first type of quantum simulators exploits the fact that alkaline earth atoms have an even number of electrons in the outer shell. The total electron spin is then zero and, hence, there is no coupling between the nuclear and the electronic spin. This leads to degenerate hyperfine levels of the atoms with degeneracy $N=2 I+1$ for a given value of the nuclear spin $I$. The largest number of degenerate states for this type of experiments is realized by ${ }^{87} \mathrm{Sr}$ with nuclear spin $I=9 / 2$, leading to $N=10$ degenerate levels. In the experiments, these levels can be addressed and populated individually, so that for a setup using ${ }^{87} \mathrm{Sr}$ one can realize $N \leq 10$ degrees of freedom (or 'flavors') per atom. When two of the so prepared atoms scatter, one finds that the population of these levels remains unchanged, which directly leads to a realization of $\mathrm{SU}(\mathrm{N})$ symmetric versions of the fermionic Hubbard model (1.8) [66]. This is very interesting, since for the first time this is a way to realize in a controlled way systems with up to $N=10$ on various types of lattice geometries. Experimental groups have reported the realization of degenerate quantum gases of such alkaline earth atoms, see e.g., [242-246] (for a recent review on the present status of experimen- 
tal and theoretical progress see [247]). Interesting for experiments is also that, similar to Pomeranchuk cooling [248], the larger number of degrees of freedom per site leads to a stronger cooling effect than in $\mathrm{SU}(2)$ systems [109, 249, 250] (see also my own contribution [SRM8]). It appears easier to reach temperature regimes relevant for the realization of interesting quantum many body states when increasing $N$, and first experiments support this prediction [244]. From the theoretical side, SU(N) systems have been considered in the past as an interesting generalization of the standard $\mathrm{SU}(2)$ Hubbard or Heisenberg model, and a number of numerical studies for low-D systems is available, e.g., [85, 87, 91, 96, 99, 101, 106, 116, 122, 134, 251] and my own contribution [SRM22] (attached in the second part of the thesis). A lot of interest has been created by the possibility of observing spin-nematic states $[54,56]$ (see chapter 1), generalizations of the AKLT valence-bond-solid state [111, 122, 127-134], and the possible realization of a chiral spin liquid state $[135,136,252,253]$. In particular the latter one has been proposed to be realizable in these experiments for large enough values of $N$ [132]. It might well be within reach since the experiments can realize up to $N=10$. In my contribution [SRM22] (attached in the second part of the thesis), we address $\mathrm{SU}(\mathrm{N})$ Hubbard chains at commensurate fillings for $N \leq 4$ using the DMRG and at large $N$ using Bethe ansatz (which gives an approximate solution for $N>2$ ). The results show the behavior of various observables and indicate that for $U / t>10$ a description in terms of a $\mathrm{SU}(\mathrm{N})$ Heisenberg model (1.9) becomes reliable. This is interesting since for the experiments it is more favorable to be at smaller values of $U / t$, and our findings can hence guide experimental setups aiming for the realization of $\mathrm{SU}(\mathrm{N})$ spin physics. Finally, using the fidelity susceptibility (1.4), we obtain indications that the transition from a metallic to a Mott insulating phase is at finite values of $U / t$ for $N>2$, confirming previous findings from Quantum Monte Carlo and bosonization approaches [85]. However, as further discussed in my contribution [SRM20], it later turned out that one probably needs a more elaborate finite size scaling in order to obtain the transitions with a high accuracy. At the end of this section, I want to briefly mention a recent important development with ultracold alkaline earth atoms, namely the realization of an optical lattice atomic clock with Sr atoms which has a stability of the order of $10^{-18}$ [254]. These approaches exploit the fact that in an optical lattice a large number of atoms is addressed at the same time, helping in controlling the stability and enabling further developments of atomic clocks. 


\subsection{Ultracold Polar Molecules for Studying Quantum Magnetism and Superconduc- tivity}

The second type of quantum simulator I want to highlight is somewhat unusual in the sense that it is not using atoms as building blocks, but ultracold polar molecules [255-268] (see also the viewpoint [269]). The creation of these molecules at temperatures on the $\mathrm{nK}$ scale is a rather intriguing recent development: using STIRAP techniques ('stimulated Raman adiabatic passage', see $[268,270]$ and references therein), the experimental groups have succeeded to subsequently transform two initially free atoms cooled to $\mathrm{nK}$ temperatures into a bound state, and then transfer this highly excited state to the energetically lowest molecular bound state. This development shows how, in principle, chemical reactions at such low temperatures can be controlled step by step [268].

These molecules have various interesting properties. For example, the ones formed in the JILA experiment - KRb - [257] are chemically reactive [261], but due to their fermionic nature, scattering is due to p-wave collisions and affected by the centrifugal barrier so the reaction rate depends strongly on the relative orientation of the molecular axis [261, 263]. When confining them to a plane and aligning the axis parallel to each other, they are practically chemically inert, so that stable setups are obtained in which life times of the order of $25 \mathrm{~s}$ have been measured [271]. Other species (e.g., NaK) are always chemically inert, which might be advantageous for certain experiments. It is possible to form fermionic (e.g., the aforementioned $\mathrm{KRb}$ ) as well as bosonic molecules (e.g., LiCs [258]). Most prominently, the fact that the molecules are heteronuclear introduces a rather large dipole moment which couples to external electrical fields (and which can be used to align them in the aforementioned setups). Most interestingly in the context of quantum simulation, the dipolar interaction can be directly used to emulate spin exchange, see the review articles [268, 272] and references therein, and my own contributions [SRM10] (attached in the second part of the thesis) and [SRM24] which discuss how to realize Heisenberg-like (1.1) and t-J-like (1.7) models. This is achieved by populating two (or more) rotational eigenstates of the molecules, forming a subspace of two (or more) states per molecule. When confining them onto an optical lattice, each lattice site is now representative for a twolevel system as in a $S=1 / 2$ spin system (or $S>1 / 2$ when populating more than two rotational eigenstates). The energy scales are typically such that the population of these levels is stable on the time scale of the experiments, 
so that in a good approximation one can treat the systems as $S \geq 1 / 2$ particles. The dipolar interaction between the molecules now directly leads to an interacting spin system [SRM10, SRM24]. The terms of the effective model are identified by mapping the dipole operator for the interaction between the molecules onto this subspace, leading to two-body interaction terms decaying as distance cube. In the case of doping (i.e. less than one particle per site), the molecules can hop between neighboring sites of the optical lattice, and when restricting the population of the optical lattice to one molecule per site, one obtains a generalization of the t-J-model Eq. (1.7),

$$
\begin{aligned}
& \mathcal{H}^{t J V W}=-t \sum_{i, \sigma}\left[f_{i, \sigma}^{\dagger} f_{i+1, \sigma}+\text { h.c. }\right] \\
& +\sum_{j>i} \frac{1}{|i-j|^{3}}\left[\frac{J_{\perp}}{2}\left(S_{i}^{+} S_{j}^{-}+S_{i}^{-} S_{j}^{+}\right)+J_{z} S_{i}^{z} S_{j}^{z}\right. \\
& \left.\quad+V n_{i} n_{j}+W n_{i} S_{j}^{z}\right] .
\end{aligned}
$$

Note that the kinetic term is short-ranged since hopping is only possible between neighboring lattice sites. However, the two-body interaction terms are all long-ranged since they originate from the dipolar interaction between the molecules. Also note that here we have chosen the fermionic variant. However, since it is possible to create bosonic molecules, it is also possible to realize a bosonic version of this model. In the context of $\mathrm{KRb}$, the chemical reactivity does not allow for double occupancy of a lattice site; via a quantum Zeno effect, [273] (see also my contribution [SRM24]) it is expected that the high reactivity leads to a suppression of the hopping to an already occupied site, so that double occupancies are excluded. It is also possible to consider Hubbard-like on-site interaction terms [SRM24] which can be tuned to large values so that again double occupancies are excluded. It therefore appears possible that the experiments can realize in a good approximation this extension of the t-J model. In a recent contribution, the JILA group has demonstrated that, indeed, the spin exchange part of (4.1) is realized in ongoing experiments [274]. This observation is based on a prediction from my own contribution [SRM6] (attached in the second part of the thesis), which I will address again later.

Let me emphasize that in contrast to the usual t-J model, this effective Hamiltonian is not the result of a strong coupling expansion of the Hubbard model, but is obtained directly from the interaction between the particles. The most eminent differences to the usual t-J model are (i) the long-range interactions, (ii) the ability to tune independently all parameters by applying 
microwaves and dc electrical fields, and (iii) the possibility to tune away from $\mathrm{SU}(2)$ symmetry. The $W$-term is rather peculiar and not easily obtained in a condensed matter context (it has been discussed in the context of variants of the 'Bariev-model' and of exactly solvable variants of the t-J model, but it is unknown to me whether a realization in experimental setups or materials has been discussed before; see, e.g., [275-281]). It is also possible to make the interaction direction dependent, which allows for the realization of compass-like models (see my own contribution [SRM5] attached in the second part of this thesis). One expects a rather rich behavior of this model. In my contribution [SRM10] (attached in the second part of the thesis), we introduce the effective model and discuss the ground state phase diagram in the case which appears to be the easiest realization in experiment and treatable with DMRG, namely the $\mathrm{t}-\mathrm{J}_{\perp}$ chain. In this case, all parameters are tuned to zero other than the hopping and the $\mathrm{J}_{\perp}$ term. Generally speaking, the phase diagram of the usual t-J chain is realized (see my own contribution [SRM26] for a recent DMRG study). However, when working in units of the hopping $t \equiv 1$, the superconducting phase is enhanced, and also the spin gap which in this case measures the binding strength of the pairs of fermions and hence the stability of the superconducting phase is largely enhanced by tuning away from the $\mathrm{SU}(2)$ symmetry of the Hubbard model and setting to zero the density-density interaction, $V=0$. The question arises if this observation persists in other configurations and higher dimensions, which is a topic presently further pursued. My contribution [SRM5] (attached in the second part of the thesis) shows that symmetry protected topological phases (see the next section) survive in the presence of long-range interactions on two-leg ladder systems.

The energy scales for the molecules are larger than the ones in other proposals for emulating super-exchange interactions with ultracold atoms [282], which should facilitate the realization of interesting many body states in the experiments. This is encouraging for future emulations of quantum magnetism and of variants of the t-J-model in these experiments. For the moment, reaching low enough temperatures and entropies is an ongoing challenge. However, there is a way to probe spin exchange by going out of equilibrium as demonstrated in my contribution [SRM6] (attached in the second part of the thesis). In the experiments presented in [274], the molecules are prepared in a state which corresponds to all the spins being aligned parallel to each other, e.g., in the $x$-direction, and the optical lattice is so deep that tunneling is suppressed. The time evolution of this initial state is trivial if two-body interactions are absent, but shows oscillatory behavior in the case of interactions. The experiments show a decay of the amplitude with time which can 
be understood as decoherence, but on top of that, oscillations with periods in agreement with the prediction from the effective spin model (i.e., (4.1) in the limit of deep lattices in which tunneling is suppressed, and in the limit of weak electrical fields, in which case only $J_{\perp} \neq 0$ ) are observed, providing a strong indication for the realization of spin exchange in these setups.

In the next chapter I will revisit the ability for controlling the non-equilibrium dynamics in quantum simulators and discuss interesting features of the nonequilibrium dynamics of quantum many body states. In the remaining part of this chapter, I will address recent developments in characterizing quantum phases which have topological properties.

\subsection{Topological Phases}

States of matter are usually characterized by the Landau paradigm of phases and phase transitions in which a continuous phase transition and the associated phases are obtained by the spontaneous breaking of one (or more) symmetries of the Hamiltonian and the emergence of a local order parameter [283] (i.e. for quantum systems a local observable exists which could be zero by the symmetries of the system, but retains a finite value in the thermodynamic limit due to the spontaneous symmetry breaking). This paradigm has been the framework for understanding phases of matter and phase transitions, until in the 1980s experiments discovered the integer [284] and later the fractional quantum Hall effect [285, 286] which possess transitions between states with different conductivities which apparently are not associated to the spontaneous breaking of symmetries. Subsequently, and also motivated by the discovery of high-temperature superconductivity [3], a new type of 'order' was proposed whose phenomenology is not due to the finiteness of some local order parameter, but in which the phases are characterized by global characteristics, like the degeneracy of the ground state or entanglement of the system. This type of order has been coined topological order [67, 287, 288] since the behavior is captured by topological field theories [289]. The main characteristics of topologically ordered phases are the presence of degenerate ground states, of gapless edge states, and the characterization in terms of topological invariants which are integer numbers capturing 'topological' properties of the system and which vary in the different phases. One characteristic of topological phases is that they do not change under continuous deformations of the system (i.e., the topological invariant does not change unless one hits a critical point at which the system experiences singular be- 
havior) and are, hence, protected against local perturbations like noise. This makes these states very interesting for quantum computation in which one of the biggest challenges is to protect the entanglement between qubits from decoherence effects due to local noise induced by the environment. This approach is coined topological quantum computation and is described in the review article [67].

At the present, obtaining a complete characterization of topological phases is an ongoing topic of research. However, one can use the following approach to distinguish between gapped phases [290, 291]: a gapped quantum phase is characterized by ground states of Hamiltonians which can be smoothly deformed into each other without closing the gap. An illustrative example is the $S=1$ BLBQ chain (3.1), which at zero magnetic field displays a gapped phase for $-\pi / 4<\theta<\pi / 4$; even though at $\theta=0$ the Hamiltonian looks much simpler, the system in this parameter range is in the same phase since the gap closes only at the endpoints of this region. This property can be rephrased by saying that two ground states belong to the same phase if they are related by a local unitary transformation. Since local unitary transformations can only change local entanglement properties but not global ones, states in the same topological phase are characterized by the same 'longrange entanglement'. Based on these considerations, the following gapped phases can be identified:

1. Phases with 'short-range entanglement':

(a) Topologically 'trivial' product states.

(b) Symmetry protected topological phases (SPT). In these phases, local unitary transformations exist which preserve the symmetry of the state. Short-range entangled phases in which such a symmetry is broken are well described by Landau theory. Note that phases without local order parameter can still belong to different SPT phases if they are characterized by different symmetries, even though in Landau classification they would belong to the same 'disordered' phase.

2. 'True' topological order with 'long-range' entanglement, existing only in spatial dimensions $D \geq 2$ [290]. These phases are characterized by anyonic fractionalized excitations, which obey a generalized quantum statistics and are neither fermions nor bosons [67].

It is possible to characterize topological order by considering entanglement properties [292, 293], and tensor-network approaches have been introduced (see, e.g. [294] and the viewpoint [295]). While it is possible to investigate for 
'true' topological order in 2D using the DMRG (for recent examples on the kagome lattice see [69-71]), in this thesis we focus on SPT phases which are often encountered in 1D. My contribution [SRM7] is based on the approach presented in [296] (and references therein) and demonstrates how topological invariants in terms of the winding number of Green's functions can be computed in the presence of interactions, see [297] for the effect of interactions in 1D on fermionic topological phases. In my contribution [SRM5] (attached in the second part of the thesis), we address the question for the existence of SPT phases in the presence of long-range interactions, as realized in quantum simulators with polar molecules, e.g., Eq. (4.1). An SPT phase can numerically be detected by identifying an excitation gap, zero local order parameters, and degeneracy of the entanglement spectrum [298] which is given by the eigenvalues of the Schmidt decomposition Eq. (2.4). As discussed in $[293,299]$, in an SPT phase all states of the entanglement spectrum are nontrivially degenerate due to the symmetry in the system. Other indications for topological properties can be obtained from diagonalizing transfer matrices from which one can obtain directly the projective representations of the symmetry group [300], which can be used to further characterize SPT phases (see also [301] for a nice discussion of this aspect). According to [301-303], it is possible to distinguish between different SPT phases by applying the corresponding active operators: if the correct active operator is coupled to the edge of the system, the ground state degeneracy is lifted. As shown in my contribution [SRM5], this can indeed be used to distinguish the different SPT phases obtained from the projective representations. Numerically, it is easily seen that applying the 'wrong' active operator does not lift the ground state degeneracy, while applying the correct one leads to different energies of the ground states with a difference far greater than the numerical accuracy. This was used in my contribution [SRM5] to see whether the SPT phases in a 2-leg $S=1 / 2$ system survive when turning on the dipolar long-range interactions, as in a quantum simulation of this model with polar molecules. As can be seen, this is the case, and it will be interesting to see if other topological phases survive in the presence of long-range interactions and whether the idea of applying active operators to the edges can be used in experimental setups for the detection of SPT phases. 


\section{Chapter 5}

\section{Nonequilibrium Dynamics of Quantum Many Body Systems}

The Experiments with ultracold atomic and molecular gases discussed in the previous chapter can be performed out-of-equilibrium in a highly controlled manner $[1,2,304]$. This opened the way to experimentally study the nonequilibrium dynamics of quantum many body systems and has spurred the development of numerical methods such as the adaptive t-DMRG [189194] (see also my own contribution [SRM38]) and time-dependent numerical renormalization group schemes (NRG) [305]. My own contributions to the nonequilibrium dynamics of quantum many body systems can be grouped in the following way ${ }^{1}$ :

i) Method development: [SRM38] (noteworthy proceedings article discussing a Krylov-space version of the adaptive t-DMRG) and [SRM36, SRM37, SRM$\mathrm{PhD}$ ] (review articles and my $\mathrm{PhD}$ thesis).

ii) Fundamental questions to the nonequilibrium dynamics of quantum many body systems: relaxation and thermalization behavior, and causal structures in the propagation of information in systems with long range interactions [SRM17, SRM18] (attached in the second part of the thesis), [SRM2, SRM15, SRM42] and [SRM39] (submitted).

iii) Transport in experiments with ultracold quantum gases [SRM21, SRM23] (attached in the second part of the thesis) and [SRM32].

iv) Dynamics of quasiparticle excitations following a quantum quench (see

\footnotetext{
${ }^{1}$ Note that several papers treat multiple of the topics, so that they are mentioned in more than one point
} 
below) at short times and the formation of unconventional metastable states [SRM29, SRM13, SRM17] (attached in the second part of the thesis) and [SRM7, SRM19].

v) Approaches for using the nonequilibrium dynamics in quantum simulators for the identification of phase transitions and the validity of effective models [SRM6, SRM10, SRM20, SRM23] (attached in the second part of the thesis).

Generally speaking, the nonequilibrium behavior studied in these contributions can be characterized in two ways: A typical out-of-equilibrium situation frequently studied is a so-called quantum quench in which the time evolution of an initial state following the sudden change of one or more intrinsic parameters of the system is studied. Examples are the experimental observation of the collapse and revival of an initial BEC state [304], and experimental $[306,307]$ as well as theoretical investigations on the thermalization behavior of these systems, see, e.g., the focus issue in New Journal of Physics [308] for an overview. My contributions [SRM6, SRM10, SRM17, SRM18, SRM20, SRM23, SRM29] (attached in the second part of the thesis) and [SRM2, SRM15, SRM19, SRM39, SRM42] treat this class of nonequilibrium situations.

A second class of interesting non-equilibrium problems is realized in transport setups. The simplest realization in systems on optical lattices is the release of initially trapped particles from a trap. This has lead to the discovery of various interesting phenomena, as, e.g., the emergence of (quasi-)coherent matter waves from initial insulating states (see, e.g., [309, 310] and my contribution [SRM32]), fermionization of bosonic systems [311], the realization of the quantum distillation effect in which repulsive interactions lead to the dynamical formation of a metastable low-entropy band-insulating state (see my contribution [SRM13] attached in the second part of the thesis), and the breakdown [312] or crossover [313] to diffusive behavior in 2D systems. In a condensed matter perspective, the crossover from ballistic to diffusive motion in correlated spin systems $[195,314]$ has been treated. This is connected to more general setups in nano- and mesoscopic systems, in which either the dynamics and the steady state when applying a finite voltage bias, or the wave packet dynamics through structures (e.g., quantum dots) connected to leads is treated $[315,316]$. At the present, the effect of interactions on the transport properties in these systems is a topic of ongoing investigations, and using the t-DMRG some progress has been reported, see, e.g., [193, 317324]. My contributions [SRM13, SRM21, SRM23] (attached in the second part of the thesis) and [SRM7, SRM32] discuss various aspects of transport on optical lattices. 
A third class of interesting non-equilibrium setups is realized in experiments for ultrafast spectroscopy of condensed matter systems. In these pump-probe experiments, ultrashort laser pulses are used to excite the system and to observe its behavior during or after the excitation [325-333] (see also the Perspectives in Science [334, 335]). This is interesting from various perspectives. It has been reported that due to the excitation process interesting states are realized, and both, their characterization and the life-time of these states is, in the great, unknown. In addition, this is a system in which the interaction between light and matter can be analyzed in detail in a quantum many-body context.

Here, we will focus on the first two aspects and discuss basic features of the nonequilibrium situations treated in the publications attached in the second part of the thesis.

\subsection{Relaxation Behavior following a Quan- tum Quench}

One of the fundamental questions in statistical mechanics addresses the emergence of a thermal quasi-stationary state in the course of the time evolution of some randomly chosen initial state. This is at the heart of the ergodic hypothesis, which was introduced by Boltzmann as a posteriori justification for the description of thermodynamical properties by statistical ensembles and averages. While the dynamics for classical systems is expected to lead to a thermal state if the system is strongly chaotic in the sense of the KAMtheorem [336] (i.e., if the dynamics covers the hypersurface of constant energy in the phase space of the system in a mixing way [337]), it is a priori not clear if such a statement is true for quantum many body systems, and to which extend integrability (i.e., the existence of conserved quantities during the time evolution other than the energy, see also [338] for a discussion of this notion in the context of quantum many body systems) affects the thermalization behavior. This has been studied in many theoretical approaches, and a huge body of literature exists. Examples are [339-348] and further work mentioned below. My contribution [SRM15] was one of the first to deal with this problem using the t-DMRG providing results for the time evolution with a high accuracy for systems with up to 100 lattice sites for both, integrable and non-integrable systems (see also the right panel of Fig. 5.1 from [SRM15] for an example of relaxation behavior). My contribution [SRM17] attached in the second part of the thesis extends this study and discusses a controlled 

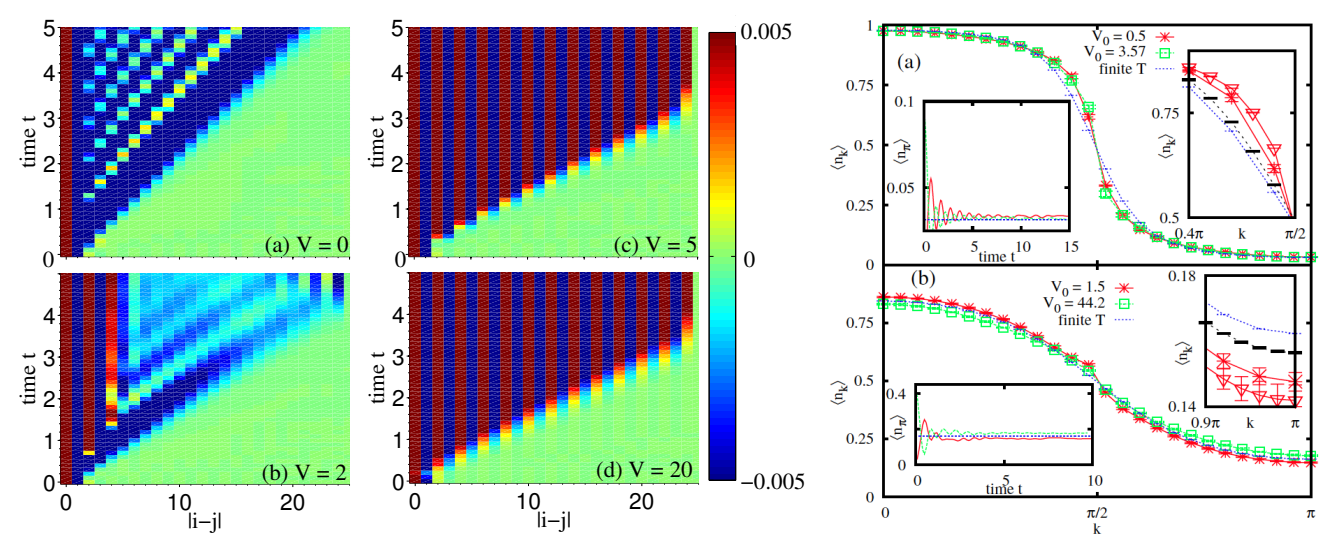

Figure 5.1: Time evolution of interacting spinless fermions following a sudden quantum quench. Left panel (taken from my contribution [SRM29] attached in the second part of the thesis; (c) by APS): time evolution of the densitydensity correlation function displaying a light-cone effect. Right panel (taken from my contribution [SRM15]; (C) by APS): comparison of the expectation value of the momentum distribution function in the quasi-stationary state to a thermal state, the explicit time evolution (left insets), and the effect of doubling the system size (right insets).

way for breaking integrability. Interestingly, on the time scales and for the system sizes treated, both studies do not provide clear signatures for thermalization, despite the breaking of integrability. It is unclear at the moment if this is due to the $1 \mathrm{D}$ character of the systems which might involve longer time scales for relaxation to the stationary state, the finiteness of the systems which makes boundary effects influence the long-time behavior, or whether there are more fundamental issues preventing these systems from relaxation to a thermal state. In [SRM17], one particular scenario is closer considered in which a metastable non-thermal state is realized which, however, eventually will relax to a thermal state for times long enough. This behavior has been coined prethermalization [349] and is investigated in a quantum many body context, e.g., in Refs. [350-353]. As discussed there and in [SRM17], the quench leads to the creation of metastable quasiparticles which describe the non-thermal prethermalized behavior, and after the quasiparticles decay or scatter to each other, the system is expected to finally reach the true steady state which is a thermal one. In [SRM17], the formation of these quasiparticles and of the prethermalization plateau is shown in a very precise way by an excellent quantitative agreement of numerical t-DMRG results to (semi-)analytical results obtained from a continuous unitary transformations 
treatment [220]. However, the turnover to thermalization is not observed, as discussed above. A second route to thermalization has been formulated in the context of Srednicki's eigenstate thermalization hypothesis (ETH) [348, 354], and current efforts aim at understanding the importance of integrability and the connection to quantum chaos in the ETH [355-358].

Note that for systems with spectrum bounded from above, it is possible to introduce the notion of 'negative absolute temperatures' [359-363]. This notion is well known since the 1950s in the context of NMR experiments [359, 360] and essentially relates to the fact that population inversion can be obtained, i.e., the higher or highest eigenstate can be substantially higher populated than the low energy eigenstates of the system, a situation which formally can be associated to a negative sign in the inverse temperature $\beta$ in a GibbsBoltzmann distribution ${ }^{2} \exp (-\beta \mathcal{H})$. This situation is similar to the setup of a laser, but here we consider population inversions which are stable in time and hence allow for a thermodynamic description. Recently, this has been studied in experiments with ultracold gases [361] and the findings there can be interpreted as negative absolute temperature states. In my contribution [SRM18] (attached in the second part of the thesis) we consider the exact time evolution of the Bose-Hubbard model (1.6) in the so-called hardcore limit $U / J \rightarrow \infty$ which in $1 \mathrm{D}$ can be treated exactly by virtue of a Jordan-Wigner transform [371]. Indeed, we identify stationary states which can be associated to negative absolute temperatures and the emergence of this state can be understood in terms of a dynamic symmetry in the Hubbard model [312]. Depending on the quench, we find that the steady state is captured by so-called generalized Gibbs ensembles [339] at positive or negative absolute temperatures, a notion further discussed in [SRM18].

While in the long-time behavior one is mainly interested in the emergence of a quasi-stationary state and its characterization, on short time scales following a quantum quench, further interesting aspects come into play. Here, I want to focus on two aspects discussed in my contributions. In [SRM29] (attached in the second part of the thesis) and [SRM2, SRM7, SRM39] the propagation of signals after a global [SRM2, SRM29, SRM29] or a local quench [SRM7]

\footnotetext{
${ }^{2}$ There is an ongoing discussion on the validity of this notion (re-)initiated by J. Dunkel and S. Hilbert [364]. The essential point of these authors' claim is that only Gibbs' formulation of the entropy leads to a consistent thermodynamical description which avoids the notion of negative absolute temperatures, while the usual formulation based on Boltzmann's notion of the entropy allows to introduce negative absolute temperatures; see the comments and replies [365-368] to Ref. [364] and further recent preprints [369, 370] and references therein. Here, I take on the standard view and refer to a state with population inversion stable in time as a state at 'absolute negative temperatures'.
} 
is described. In all cases, if the interaction is short-ranged, a 'light-cone' (see left pannel of Fig. 5.1) is found; in the presence of long-range interactions, the light-cone can get modified to a non-linear region of causal propagation of information (see [SRM2] and references therein) and for power-law interactions with an exponent smaller than the spatial dimension, instantaneous propagation of information is found. The light-cone follows a prediction by Calabrese and Cardy [372] based on conformal field theory according to which a lightcone effect in correlation functions is obtained due to scattering of entangled quasiparticles created due to the quench. This has been confirmed using the adaptive t-DMRG in [373] and my contribution [SRM29] (attached in the second part of the thesis), and the light-cone effect has in the meantime been seen in experiments on optical lattices [374]. This can be contrasted to the Kibble-Zurek mechanism [375-377] in which quenches of a finite duration to a symmetry broken state create stable topological defects separating domains in which the different vacua of the system get realized, which might be an alternative scenario also for sudden quenches (see [SRM29] attached in the second part of the thesis).

The second short-time aspect following a quantum quench lies in the probing of effective models and of phase transitions in experiments on optical lattices. As discussed in [SRM10, SRM20, SRM23], I propose to use Bloch oscillations [378] to get an estimate for phase transition points when performing the quantum simulation of a quantum many body system for obtaining its low-temperature phase diagram. The idea is that in different phases the dynamics is dominated by different types of excitations; e.g., in a superconductor, the pairs of fermions will dominate the center of mass motion, while in a metal it will be single fermions, so that by considering the amplitude and frequency of Bloch oscillations the phase transition can be estimated, see my contribution [SRM10] (attached in the second part of the thesis) for numerical findings at low fillings. Also at low fillings, [SRM20] (attached in the second part of the thesis) manages to obtain a rather good estimate of the phase transition point in a 1D Bose Hubbard system (1.6) at filling $n=1$. The amplitude of the Bloch oscillations vanishes around the phase transition point, so that it appears feasible to use the nonequilibrium dynamics as indicator for quantum critical behavior ${ }^{3}$. A further aspect of the short-time behavior of quantum quenches on optical lattices is that the dynamics can be used to identify many-body interaction terms. As proposed in my contribution [SRM6] and later identified in experiments with ultracold

\footnotetext{
${ }^{3}$ This seems to work best at low fillings, the behavior at higher fillings is more involved and I leave the question open for future research if this approach can be used successfully there.
} 
polar molecules [274], a description in terms of an effective spin model (see Sec. 4.2 for more details) leads to typical short time behavior and oscillations which have been observed in the experimental work [274] hence indicating the validity of the effective spin model in this experimental setup.

\subsection{Transport on Optical Lattices}

Interesting non-equilibrium situations can also be realized when releasing particles from a trap, applying a voltage bias, or when studying excitations which remain invariant in shape in the course of the time evolution (solitons). We will briefly revisit these three nonequilibrium situations in the context of optical lattices.

When releasing particles from an initial trapping potential (e.g., a box), one expects them to flow into the empty space and disappear eventually. However, on a lattice and in the presence of interactions, this is not necessarily true. Both, bosonic [309, 311] (see also my contribution [SRM32]) and fermionic [310] (see also my contribution [SRM13] attached in the second part of the thesis) systems have been treated, and interesting behavior is found: Particles initially forming a Fock state [309] or a trapped Mottinsulator [SRM32] dynamically create a (quasi-)coherent matter wave when released from the trap, with a wave vector which depends on the strength of the interaction. This can be envisaged to realize an atom-laser on optical lattices with tunable wavelength. When the initial density is high enough, the 'Quantum Distillation' effect takes place which is discussed in my contribution [SRM13] (attached in the second part of the thesis; see also recent experimental work on 1D bosons in the preprint [379]): in fermionic Hubbard systems (1.5) for values of the repulsive interaction $U / t$ much larger than the bandwidth and fillings $n \gtrsim 1.5$, repulsively bound pairs [380] are created which possess a lifetime $\sim U$. In the real time evolution, this is seen as an actual increase of the local density close to the maximal possible value $n=2$, while for free particles the initial state melts away, as expected. In this way, it is possible to realize a band-insulator of doubly occupied sites in a Fermi-Hubbard system with a lower entanglement entropy than the initial state. The question arises for the possibility to use this in cooling schemes in low-dimensional fermionic systems on optical lattices, but further studies at finite temperatures are needed.

Typical transport setups are obtained when attaching leads to a system and 
applying a voltage bias, which is equivalent to having an electrical field along the system or a chemical potential which varies linearly with position. In optical lattices, this can be realized by tilting the lattice and letting the gravitational force pull the particles in one direction. In a solid state system, the presence of impurities and defects leads to scattering centers which typically inhibit coherent motion and, in the presence of an external field, eventually lead to a directed net current [378]. This is not the case in a clean system as it can be realized in optical lattices: the lack of defects allows for the formation of coherent motion, and so-called Bloch oscillations are realized. Due to Bragg scattering, the center of mass performs in the presence of a constant force an oscillatory motion on the lattice with amplitude depending on the strength of the applied field and the mass of the particles. This amplitude can be smaller than a lattice spacing, so that there is no net current flowing from one end of the system to the other end. While they are an interesting phenomenon per se to study, for investigating transport in such setups in optical lattices, one needs to find other routes for creating a net current. In my contribution [SRM23] (attached in the second part of the thesis) we propose a scheme coined slinky transport, in which a periodic modulation of the optical lattice leads to a directed center of mass motion, interestingly for appropriately chosen parameters also opposite to the acting force.

An intriguing type of excitations traveling through a system are solitons which do not change their shape in the course of the time evolution. The first observation of a soliton is reported by John Scott Russell in 1834, who reported a water wave which travelled through the Union Canal in Scotland for a very long distance barely changing its shape (see, e.g., the corresponding Wikipedia entry). Solitons and solitary excitations are due to nonlinearities in the system and it is interesting to study them in the context of ultracold gases. Typically, soliton excitations are realized in Bose-Einstein condensates (BEC) [381-385] in a continuum (see [386, 387] and my contribution [SRM21] for soliton solutions on a lattice).

Interestingly, in addition to the dark soliton which is usually observed in the continuum case of BECs and understood in terms of the Gross-Pitaevski equation [388, 389], also bright solitons can be realized [390]. The question arises, whether these solutions survive quantum fluctuations and the effect of a lattice. In my contribution [SRM21] (attached in the second part of the thesis) we address this question and show that on a time scale accessible to experiments this is indeed the case. Furthermore, it is not crucial that the initial perturbation has exactly the predicted shape of the solitonic solution, but that the correct phase of the wave function is imprinted onto the initial 
state. This will help in the search of this type of solitons in optical lattice realizations of the proposed setup. 


\section{Chapter 6}

\section{Outlook}

In this thesis, I have summarized recent developments in the investigation of many-body effects in strongly interacting quantum systems. I highlighted three aspects: 1) the realization of quantum many-body behavior in quantum magnetic materials, in particular their behavior in strong magnetic fields. 2) the approach to unconventional many-body states in so-called quantum simulators with ultracold atomic and molecular gases. 3) the non-equilibrium dynamics which demonstrates various interesting effects and also addresses fundamental questions to the foundations of statistical mechanics, in particular concerning the relaxation behavior to a possible thermal state. From the theoretical side, it has been proven very useful to apply numerical approaches to the problems. One particularly useful method is the DMRG and its generalization in terms of MPS and PEPS allowing for the formulation of more efficient algorithms e.g. for systems with periodic boundary conditions and in higher dimension, as well as the adaptive t-DMRG which allows to treat the nonequilibrium dynamics of low-dimensional quantum many body systems with a high accuracy on short to intermediate time scales. In this thesis, various aspects of the physics of the aforementioned topics is addressed mainly using the DMRG and its variants, resulting in the uncovering of a rich bouquet of interesting behavior.

The realization of unconventional states of matter in strongly correlated systems remains an interesting topic of ongoing research. Future studies, e.g., in the context of pump-probe experiments will reveal further interesting behavior due to quantum effects. Particularly interesting are the uncovering of new effects in situations out-of-equilibrium, the investigation of quantum magnetism in quantum simulators, and the search for topological phases, which form a new paradigm for the characterization of quantum phases. 
Both, theoretical and experimental efforts will contribute to the wealth of behavior and it is worth to look forward to intriguing discoveries in the years to come. 


\section{List of Own Contributions}

In the following a complete list of my publications at the time of finalizing this thesis. The articles marked by * are the ones attached in the second part of the thesis.

\section{Publications in Letter Journals, Rapid Communications, and Editors' Suggestions (chronological order):}

SRM1 A.C. Tiegel, S.R. Manmana, T. Pruschke, and A. Honecker, Matrix product state formulation of frequency-space dynamics at finite temperatures,

Phys. Rev. B (rapid communication) 90, 060406(R) (2014).

SRM2 J. Eisert, M. van den Worm, S.R. Manmana, and M. Kastner, Breakdown of quasi-locality in long-range quantum lattice models, Phys. Rev. Lett. 111, 260401 (2013).

* SRM3 Y.H. Matsuda, N. Abe, S. Takeyama, H. Kageyama, P. Corboz, A. Honecker, S.R. Manmana, G.R. Foltin, K.P. Schmidt, and F. Mila, Magnetization of $\mathrm{SrCu}_{2}\left(\mathrm{BO}_{3}\right)_{2}$ in ultrahigh magnetic fields up to $118 \mathrm{~T}$, Phys. Rev. Lett. 111, 137204 (2013).

SRM4 F. Michaud, S.R. Manmana, and F. Mila, Realization of higher Wess-Zumino-Witten models in spin chains, Phys. Rev. B (rapid communication) 87, 140404(R) (2013);

Editors' Suggestion.

* SRM5 S.R. Manmana, E.M. Stoudenmire, K.R.A. Hazzard, A.M. Rey, and A.V. Gorshkov,

Topological phases in ultracold polar-molecule quantum magnets, Phys. Rev. B (rapid communication) 87, 081106(R) (2013). 
* SRM6 K.R.A. Hazzard, S.R. Manmana, M. Foss-Feig, and A.M. Rey, Far from equilibrium quantum magnetism with ultracold polar molecules, Phys. Rev. Lett. 110, 075301 (2013); research highlight at JILA.

SRM7 S.R. Manmana, A.M. Essin, R.M. Noack, and V. Gurarie, Topological invariants and interacting one-dimensional fermionic systems, Phys. Rev. B 86, 205119 (2012) - Editors' Suggestion.

SRM8 L. Bonnes, K.R.A. Hazzard, S.R. Manmana, A.M. Rey, and S. Wessel, Adiabatic loading of one-dimensional $S U(N)$ alkaline earth fermions in optical lattices, Phys. Rev. Lett. 109, 205305 (2012); research highlight at JILA.

SRM9 F. Michaud, F. Vernay, S.R. Manmana, and F. Mila, Antiferromagnetic Spin-S Chains with Exactly Dimerized Ground States, Phys. Rev. Lett. 108, 127202 (2012).

* SRM10 A.V. Gorshkov, S.R. Manmana, G. Chen, J. Ye, E. Demler, M.D. Lukin, and A.M. Rey, Tunable Superfluidity and Quantum Magnetism with Ultracold Polar Molecules,

Phys. Rev. Lett. 107, 115301 (2011); featured as Synopsis in Physics and as research highlight at JILA. DARPA-OLE best theoretical paper award.

* SRM11 S.R. Manmana, J.-D. Picon, K.P. Schmidt, and F. Mila, Unconventional magnetization plateaus in a Shastry-Sutherland spin tube, Europhysics Letters (EPL) 94, 67004 (2011).

* SRM12 S.A. Zvyagin, E. Čižmár, M. Ozerov, J. Wosnitza, R. Feyerherm, S.R. Manmana, and F. Mila,

Field-Induced Gap in a Quantum Spin-1/2 Chain in a Strong Magnetic Field, Phys. Rev. B (rapid communication) 83, 060409(R) (2011).

* SRM13 F. Heidrich-Meisner, S.R. Manmana, M. Rigol, A. Muramatsu, A.E. Feiguin, and E. Dagotto, Quantum distillation: dynamical generation of low-entropy states of strongly correlated fermions in an optical lattice, Phys. Rev. A (rapid communication) 80, 041603(R) (2009). 
* SRM14 S.R. Manmana and F. Mila, Torque anomalies at magnetization plateaux in quantum magnets with Dzyaloshinskii-Moriya interactions, Europhysics Letters (EPL) 85, 27010 (2009).

SRM15 S.R. Manmana, S. Wessel, R.M. Noack, and A. Muramatsu, Strongly correlated fermions after a quantum quench, Phys. Rev. Lett. 98, 210405 (2007).

\section{Regular Articles in Peer Reviewed Journals (chronolog- ical order):}

* SRM16 G.R. Foltin, S.R. Manmana, and K.P. Schmidt, Exotic magnetization plateaus in a quasi-two-dimensional Shastry-Sutherland model,

Phys. Rev. B 90, 104404 (2014).

* SRM17 F.H.L. Essler, S. Kehrein, S.R. Manmana, and N.J. Robinson, Quench Dynamics in a Model with Tuneable Integrability Breaking, Phys. Rev. B 89, 165104 (2014).

* SRM18 S. Mandt, A.E. Feiguin, and S.R. Manmana, Relaxation towards negative temperatures in bosonic systems: Generalized Gibbs ensembles and beyond integrability, Phys. Rev. A 88, 043643 (2013).

SRM19 S. Li, S.R. Manmana, A.M. Rey, R. Hipolito, A. Reinhard, J.-F. Riou, L.A. Zundel, and D.S. Weiss, Self-trapping dynamics in a 2D optical lattice, Phys. Rev. A 88, 023419 (2013).

* SRM20 J. Carrasquilla, S.R. Manmana, and M. Rigol, Scaling of the gap, fidelity susceptibility, and Bloch oscillations across the superfluid to Mott insulator transition in the one-dimensional BoseHubbard model,

Phys. Rev. A 87, 043606 (2013). 
* SRM21 C.P. Rubbo, I.I. Satija, W.P. Reinhardt, R. Balakrishnan, A.M. Rey, and S.R. Manmana,

Quantum dynamics of solitons in strongly interacting systems on optical lattices,

Phys. Rev. A 85, 053617 (2012); one figure selected for Phys. Rev. A Kaleidoscope.

* SRM22 S.R. Manmana, K.R.A. Hazzard, G. Chen, A.E. Feiguin, and A.M. Rey,

$S U(N)$ magnetism in chains of ultracold alkaline-earth-metal atoms: Mott transitions and quantum correlations,

Phys. Rev. A 84, 043601 (2011); referred to in research highlight at JILA.

* SRM23 C.P. Rubbo, S.R. Manmana, B.M. Peden, M.J. Holland, and A.M. Rey, Resonantly Enhanced Tunneling and Transport of Ultracold Atoms on Tilted Optical Lattices,

Phys. Rev. A 84, 033638 (2011).

SRM24 A.V. Gorshkov, S.R. Manmana, G. Chen, E. Demler, M.D. Lukin, and A.M. Rey,

Quantum magnetism with polar alkali-metal dimers,

Phys. Rev. A 84, 033619 (2011); featured by Synopsis in Physics and as research highlight at JILA.

* SRM25 S.R. Manmana, A.M. Läuchli, F.H.L. Essler, and F. Mila, Phase diagram and continuous pair-unbinding transition of the bilinearbiquadratic $S=1$ Heisenberg chain in a magnetic field, Phys. Rev. B 83, 184433 (2011).

SRM26 A. Moreno, A. Muramatsu, and S.R. Manmana, Ground-State Phase Diagram of the $1 D$ t-J model, Phys. Rev. B 83, 205113 (2011).

* SRM27 F. Michaud, T. Coletta, S.R. Manmana, J.-D. Picon, and F. Mila, Frustration induced plateaux in $S=1 / 2$ Heisenberg spin ladder systems, Phys. Rev. B 81, 014407 (2010).

* SRM28 I. Rousochatzakis, S.R. Manmana, A.M. Läuchli, B. Normand, and F. Mila,

Dzyaloshinskii-Moriya anisotropy and non-magnetic impurities in the $s=1 / 2$ kagome system $\mathrm{ZnCu}(\mathrm{OH})_{6} \mathrm{Cl}_{2}$,

Phys. Rev. B 79, 214415 (2009). 
* SRM29 S.R. Manmana, S. Wessel, R.M. Noack, and A. Muramatsu, Time evolution of correlations in strongly interacting fermions after a quantum quench,

Phys. Rev. B 79, 155104 (2009).

SRM30 S. Miyahara, J.-B. Fouet, S.R. Manmana, R.M. Noack, H. Mayaffre, I. Sheikin, C. Berthier, and F. Mila, Uniform and staggered magnetizations induced by Dzyaloshinskii-Moriya interactions in isolated and coupled spin 1/2 dimers in a magnetic field, Phys. Rev. B 75, 184402 (2007).

SRM31 The ALPS collaboration (A.F. Albuquerque, et al. ), The ALPS project release 1.3: open source software for strongly correlated systems,

Proceedings of the 17th International Conference on Magnetism, J. Magn. Mag. Mat. 310, 1187 (2007).

SRM32 K. Rodriguez, S.R. Manmana, M. Rigol, R.M. Noack, and A. Muramatsu, Coherent matter waves emerging from Mott-insulators,

New J. Phys. 8, 169 (2006). Invited paper to the focus issue Cold Atoms in Optical Lattices.

SRM33 M. Rigol, S.R. Manmana, A. Muramatsu, R.T. Scalettar, R.R.P. Singh, and S. Wessel,

Comment on Novel Superfluidity in a Trapped Gas of Fermi Atoms with Repulsive Interaction Loaded on an Optical Lattice,

Phys. Rev. Lett. 95, 218901 (2005).

SRM34 The ALPS collaboration (F. Alet, et al. ),

The ALPS Project: Open Source Software for Strongly Correlated Systems,

J. Phys. Soc. Jpn. Suppl. 74, 30 (2005).

SRM35 S.R. Manmana, V. Meden, R.M. Noack, and K. Schönhammer, Quantum Critical Behavior of the One-Dimensional Ionic Hubbard Model, Phys. Rev. B 70, 155115 (2004). 


\section{Review Articles, Method Development:}

SRM36 R.M. Noack, S.R. Manmana, S. Wessel, and A. Muramatsu, Studying Time-Dependent Quantum Phenomena with the Density-Matrix Renormalization Group,

Conference Proceedings for the Heraeus summer school Computational Many-Particle Physics in Greifswald, Springer Lecture Notes in Physics 739, 637652 (2008).

SRM37 R.M. Noack and S.R. Manmana,

Diagonalization- and Numerical Renormalization-Group-Based Methods for Interacting Quantum Systems,

Lecture Notes for the Conference Proceedings IX. Training Course in the Physics of Correlated Electron Systems and High-Tc Superconductors, AIP Conference Proceedings 789, 93163 (2005).

SRM38 S.R. Manmana, A. Muramatsu, and R.M. Noack, Time Evolution of One-Dimensional Quantum Many Body Systems, Conference Proceedings IX. Training Course in the Physics of Correlated Electron Systems and High-Tc Superconductors, AIP Conference Proceedings 789, 269278 (2005).

\section{Submitted:}

SRM39 K.R.A. Hazzard, M. van den Worm, M. Foss-Feig, S.R. Manmana, E. Dalla Torre, T. Pfau, M. Kastner, and A.M. Rey, Quantum correlations and entanglement in far-from-equilibrium spin systems, arXiv:1406.0937, submitted to Phys. Rev. A (2014).

\section{Conference Proceedings:}

SRM40 M. Ozerov, J.Wosnitza, E. Čižmár, R. Feyerherm, S.R. Manmana, F. Mila, and S.A. Zvyagin,

Field-Induced Gap in the Spin-1/2 Heisenberg Chain Compound CuPyrimidine Dinitrate: ESR Studies in Magnetic Fields up to 63 T, Journal of Low Temperature Physics 170, 268-273 (2013). 
SRM41 C. Lavalle, S.R. Manmana, S. Wessel, and A. Muramatsu, Monte Carlo Simulations of Strongly Correlated and Frustrated Quantum Systems,

Springer High Performance Computing in Science and Engineering 06 Transactions of the HLRS 2006, 137151 (2007).

SRM42 S.R. Manmana, A. Muramatsu, and R.M. Noack, Collapse and Revival Starting from a Luttinger Liquid, Conference Proceedings, ESF Exploratory Workshop Effective Models for Low-Dimensional Strongly Correlated Systems, AIP Conference Proceedings 816, 198203 (2006).

\section{Theses:}

SRMPhD S.R. Manmana, Nonequilibrium Dynamics of Strongly Correlated Quantum Systems, PhD thesis University of Stuttgart (2006).

SRMDiploma S.R. Manmana, Vom Bandisolator zum Mott-Hubbard-Isolator in einer Raumdimension, Diploma thesis University of Göttingen (2002). 


\section{Part II}

\section{Selected Results Published in Refereed Journals}



In the second part of the thesis, some of my published work is presented. The following topics are covered:

I) Quantum Critical Behavior and Unconventional Phases.

II) Quantum Simulations.

III) Non-Equilibrium Dynamics.

Each of the topics is presented in a separate chapter. Note that several of the publications cover more than one of these topics (e.g., PRL 110, 075301 (2013) treats quantum simulation in a non-equilibrium situation). In these cases, the paper is assigned to the topic which reflects best the main purpose of the publication. A short description of the content and of my contribution to each paper is given before the manuscript is shown. It is understood that for all papers I was involved in writing the manuscript and in the discussions/interpretations underlying the publication.

Note added to the published version of the thesis: In order to avoid conflict with copyright, the pdf-versions of the publications are not provided here. Instead, for each publication a link to the published version as well as a link to the arXiv-preprint version is given. The preprint-version is freely available and very similar to the published article, but can have minor differences to the published one. 


\section{Chapter 7}

\section{Quantum Critical Behavior and Unconventional Phases}

In this chapter, I present published results related to quantum critical behavior (i.e., phase transitions which take place at temperature $T=0 K$ ). In the first section, the behavior of various spin systems in external magnetic fields is treated. The motivation for treating these systems comes mostly from modeling quantum magnetic materials, noteworthy are the finding of unconventional phases and phase transitions. In the second section, more realistic modeling of quantum magnetic materials is aimed at by considering the effect of spin-orbit coupling treated as Dzyaloshinskii-Moriya interaction (3.2). In the third section, the quantum critical behavior in Bose Hubbard models (1.6) and in $\mathrm{SU}(\mathrm{N})$ symmetric fermionic Hubbard models (1.8) is revisited by applying quantum information approaches. Amongst others, the quantum critical behavior is addressed by considering the fidelity susceptibility (1.4) across the phase transition as indicator for the critical point. Different to the previous sections, the motivation for treating these systems comes mostly from their realization with ultra cold atomic gases on optical lattices. The focus of these papers therefore rather lies in providing insights for future experimental investigations on these systems. 


\subsection{Spin Systems In Magnetic Fields}

S.R. Manmana, J.-D. Picon, K.P. Schmidt, and F. Mila, Unconventional magnetization plateaus in a Shastry-Sutherland spin tube, Europhysics Letters (EPL) 94, 67004 (2011).

This is the first of three papers in this thesis which treat Shastry-Sutherland systems. Here, a quasi-one-dimensional version of the two-dimensional ShastrySutherland lattice is analyzed, which allows to better control the DMRG calculations. By combining a PCUTs approach and the DMRG, we can characterize the complete phase diagram of the system in a magnetic field. I had a central role in this project, the discussions and the writing of the paper. My contributions enclose the various DMRG results, in particular the phase diagram and the uncovering of the unconventional Mott-insulator at $M=1 / 5$.

Link to the published version:

http://dx.doi.org/10.1209/0295-5075/94/67004

Link to the preprint version:

https://arxiv.org/abs/1003.1696 
Y.H. Matsuda, N. Abe, S. Takeyama, H. Kageyama, P. Corboz, A. Honecker, S.R. Manmana, G.R. Foltin, K.P. Schmidt, and F. Mila, Magnetization of $\mathrm{SrCu}_{2}\left(\mathrm{BO}_{3}\right)_{2}$ in ultrahigh magnetic fields up to $118 \mathrm{~T}$, Phys. Rev. Lett. 111, 137204 (2013).

This paper presents measurements and theoretical results for the magnetization curve of the quantum magnetic material $\mathrm{SrCu}_{2}\left(\mathrm{BO}_{3}\right)_{2}$, which is described by the two-dimensional Shastry-Sutherland lattice, up to extremely high magnetic fields of $118 \mathrm{~T}$. The paper combines a state-of-the-art experimental study with state-of-the-art numerical results which show very good agreement if the ratio of inter-dimer couplings $J^{\prime}$ to intra-dimer couplings $J$ takes the specific value $J^{\prime} / J=0.63$. This is mainly achieved by an iPEPS approach by one of the authors (P. Corboz), which by construction is suited to treat two-dimensional systems directly in the thermodynamic limit (see Ch. 2). The difficulties of this approach are that one needs to find the correct unit cell of the ground state, and that it is difficult to control convergence. Therefore, it is important to compare the iPEPS results to other numerical approaches. In this paper, this is done by comparing to exact diagonalizations, unitary transformations (PCUTs), and the DMRG; different to the previous paper, here the DMRG is applied to the full $2 \mathrm{D}$ case. This is a challenge since the DMRG works best for one-dimensional systems with open boundary conditions, while here we need to treat the full twodimensional case with periodic boundary conditions in order to be able to compare to the iPEPS results. Interestingly, the results for the magnetization curves obtained from the various approaches are in very good agreement within error bars, which supports the picture emerging from the iPEPS approach. My contributions to this paper are the DMRG results which for this situation are challenging to obtain, detailed comparisons to the other numerical approaches and the detailed discussion of the DMRG findings in the supplementary material.

Link to the published version:

http://dx.doi.org/10.1103/PhysRevLett.111.137204

Link to the preprint version (including supplementary material):

https://arxiv.org/abs/1308.4151 
G.R. Foltin, S.R. Manmana, and K.P. Schmidt, Exotic magnetization plateaus in a quasi-two-dimensional Shastry-Sutherland model,

Phys. Rev. B 90, 104404 (2014).

In complement to the previous two papers, this work treats the ShastrySutherland lattice in a quasi-2D geometry. Again, a combination of the (semi-) analytical PCUTs approach and the DMRG is used to perform a detailed analysis of the system. Other than in the previous paper, here we focus on a particular tube-like geometry to facilitate computations, allowing us to obtain local structures with a very high accuracy. I had a central role in this project by providing the DMRG results, discussing the behavior and in writing the paper. In particular, the DMRG results were obtained with such a high accuracy that tiny differences (of the order of $10^{-4}$ ) to the firstly obtained PCUTs results urged the developments of more accurate effective models in the PCUTs which change the physical picture substantially.

Link to the published version:

http://dx.doi.org/10.1103/PhysRevB.90.104404

Link to the preprint version:

https://arxiv.org/abs/1405.3260 
S.R. Manmana, A.M. Läuchli, F.H.L. Essler, and F. Mila, Phase diagram and continuous pair-unbinding transition of the bilinear-biquadratic $S=1$ Heisenberg chain in a magnetic field, Phys. Rev. B 83, 184433 (2011).

This paper presents the complete phase diagram of the one-dimensional $S=1$ bilinear-biquadratic Heisenberg chain (3.1) in a magnetic field. Noteworthy are the identification of spin-nematic ('quadrupolar') phases, and of a transition line along which the central charge is $c=3 / 2$, which is a rather unconventional value and which we associate to an Ising-type pair-unbinding transition. I had a central role in this project by contributing the extensive DMRG results which allowed us to map out the phase diagram, discussions on and interpretations of the results and in writing the paper.

Link to the published version:

http://dx.doi.org/10.1103/PhysRevB.83.184433

Link to the preprint version:

https://arxiv.org/abs/1012.4518 
F. Michaud, T. Coletta, S.R. Manmana, J.-D. Picon, and F. Mila, Frustration induced plateaux in $S \geq 1 / 2$ Heisenberg spin ladder systems, Phys. Rev. B 81, 014407 (2010).

This paper presents the phase diagrams of frustrated two-leg Heisenberg spin ladder systems for values of the spin $S \geq 1 / 2$. In the strong-rung limit, effective models are derived which allow to predict the behavior for all values of $S$. These are confirmed by numerical DMRG calculations up to $S=2$, which also allowed us to treat cases beyond the strong rung limit. One of the highlights is the finding of phase transitions inside some of the magnetization plateaus.

The results discussed in this paper are mainly obtained by F. Michaud and T. Coletta, at that time both Master students in the group of Prof. F. Mila at EPFL. The contribution by F. Michaud was achieved under my supervision (co-supervision with Prof. F. Mila) and using my code. I had a central role in this project by guiding the students, in the discussions and in writing the paper.

Link to the published version:

http://dx.doi.org/10.1103/PhysRevB.81.014407

Link to the preprint version:

https://arxiv.org/abs/0907.1461 


\subsection{Frustrated Quantum Magnets with Spin- Orbit Coupling in Strong Magnetic Fields}

This section discusses published results in which the effect of spin-orbit coupling, modeled by the Dzyaloshinskii-Moriya (DM) interaction (3.2) is taken into account for obtaining a more realistic modeling of quantum magnetic materials in strong magnetic fields. The papers in this chapter focus on three aspects: first, we consider frustrated two-leg ladder systems as toy models for investigating the effect of DM interactions in magnetic torque measurements, which is a technique often used to obtain magnetization curves of correlated materials at low temperatures. Second, our theoretical results explain the behavior of low-lying excitations in the quasi-one-dimensional material $\mathrm{Cu}$ PM with substantial DM interactions. Noteworthy is the excellent agreement of the DMRG and the experimental results for the gap as a function of the external magnetic field. Third, we consider the antiferromagnetic Heisenberg model on the kagome lattice and the interplay of DM interactions and impurities. 
S.R. Manmana and F. Mila, Torque anomalies at magnetization plateaux in quantum magnets with DzyaloshinskiiMoriya interactions,

Europhysics Letters (EPL) 85, 27010 (2009).

This paper discusses frustrated two-leg ladder systems which we consider as toy models to reveal typical behavior of quantum magnetic materials in magnetic fields in the presence of anisotropies, specifically anisotropic g-tensors and DM interactions. While an anisotropic g-tensor is known to lead to a magnetic torque which can be used to determine the magnetization curve in corresponding experiments, we show that care needs to be taken when interpreting results of torque measurements in the presence of DM interactions. Interestingly, strong peak-like anomalies come into play at phase transition points at finite magnetizations, which we propose to use to obtain the endpoints of magnetization plateaus with a high accuracy. I had a central role in this project by contributing all of the results and in the writing of the paper in close collaboration with F. Mila.

Link to the published version:

http://dx.doi.org/10.1209/0295-5075/85/27010

Link to the preprint version:

https://arxiv.org/abs/0810.5329 
S.A. Zvyagin, E. Čižmár, M.Ozerov,J.Wosnitza,R.Feyerherm,S.R.Manmana, and F.Mila,

Field-Induced Gap in a Quantum Spin-1/2 Chain in a Strong Magnetic Field, Phys. Rev. B (rapid communication) 83, 060409(R) (2011).

This paper presents an experimental study and comparison to theoretical results for the quasi-one-dimensional quantum magnetic material $\mathrm{Cu}-\mathrm{PM}$ in which ESR measurements in magnetic fields up to $63 \mathrm{~T}$ are presented. Due to the DM interaction in this material, a minimum of the gap as a function of the magnetic field is obtained. We compare the experimental ESR results to numerical DMRG results for the gap and find excellent agreement for all values of the magnetic fields treated, indicating that the model Hamiltonian used to describe $\mathrm{Cu}-\mathrm{PM}$ and other, similar materials, is a valid description. My contribution to this paper is the numerical calculation of the gap compared in Fig. 3 to the experimental results and in the writing of the theoretical part.

Link to the published version:

http://dx.doi.org/10.1103/PhysRevB.83.060409

Link to the preprint version:

https://arxiv.org/abs/1010.6141 
I. Rousochatzakis, S.R. Manmana, A.M. Läuchli, B. Normand, and F. Mila, Dzyaloshinskii-Moriya anisotropy and non-magnetic impurities in the $s=$ 1/2 kagome system $\mathrm{ZnCu}_{3}(\mathrm{OH})_{6} \mathrm{Cl}_{2}$,

Phys. Rev. B 79, 214415 (2009).

This paper was motivated by NMR experiments on Herbertsmithite, a natural mineral whose lattice structure is the kagome lattice, but which contains $\sim 5 \%$ non-magnetic impurities and significant DM interactions (see introduction). By a state-of-the-art numerical calculation using exact diagonalization techniques, we analyze the behavior in the vicinity of the impurity and find that for small DM interactions the minimal building block to understand the observed behavior consists of two orthogonally coupled dimers. By increasing the value of the DM interaction, we obtain a phase transition to a semiclassical $120^{\circ}$ state. We obtain qualitatively similar behavior to experimental ESR and NMR results for values of the DM interaction which are in agreement with ESR measurements, but in disagreement with other theoretical predictions. My contributions to this work are some numerical results from exact diagonalization methods, close collaboration in obtaining and interpreting the presented numerical results, discussions and interpretations of the findings and in the writing of the manuscript.

Link to the published version:

http://dx.doi.org/10.1103/PhysRevB.79.214415

Link to the preprint version:

https://arxiv.org/abs/0903.2884 


\subsection{Quantum Information Approach}

In the last section of this chapter, I want to present two works in which quantum critical behavior was investigated using a quantum information approach by computing the fidelity susceptibility (1.4). The hope is that this quantity helps in identifying quantum critical behavior by considering small systems. As we see here, there are, however, situations in which a delicate finite size scaling can come into play, so that the results obtained for small systems need to be considered with care.

The discussions in these papers are in context of the next chapter which treats quantum simulation of strongly correlated systems. Specifically, here I present results for the Bose Hubbard model (1.6) and the fermionic SU(N) Hubbard model (1.8). Both can be realized on optical lattices, see Chs. 1 and 4 . 
S.R. Manmana, K.R.A. Hazzard, G. Chen, A.E. Feiguin, and A.M. Rey, $S U(N)$ magnetism in chains of ultracold alkaline-earth-metal atoms: Mott transitions and quantum correlations,

Phys. Rev. A 84, 043601 (2011).

This paper presents results on fermionic Hubbard chains with an enhanced $\mathrm{SU}(\mathrm{N})$ symmetry (1.8), as realizable by alkaline earth metal atoms on optical lattices. We present results for correlation functions, for up to quintuple occupancies of the sites, and for structure factors. By comparing to the Heisenberg case, we identify that for values of the interaction $U \gtrsim 10 t$ (with hopping amplitude $t$ ) for all values of $N$ the results are very well approximated by the corresponding $\mathrm{SU}(\mathrm{N})$ Heisenberg model. We also provide a comparison to Bethe ansatz, which provides an exact solution for $N=2$, but not for $N>2$. While these results are all obtained using rather traditional DMRG approaches, I want to highlight the computation of the fidelity susceptibility (FS)(1.4) which we expected to shed some light on the issue whether the transition to a Mott insulator for $N>2$ might be at $U=0$. This is difficult to answer numerically due to possible subtle behavior of the Luttinger parameters, as pointed out by J. Sólyom and collaborators [96]. The hope was that this can be resolved by considering this quantity, which in the next paper is discussed in more detail. Indeed, the FS seems to support scenarios with a transition at $U / t>0$, but according to the results of the next contribution [SRM20], probably a more elaborate finite size scaling is needed. I had a central role in this project by computing all of the numerical DMRG results for the various quantities presented, discussions and interpretations of the results and in the writing of the paper.

Link to the published version:

http://dx.doi.org/10.1103/PhysRevA.84.043601

Link to the preprint version:

https://arxiv.org/abs/1108.2327 
J. Carrasquilla, S.R. Manmana, and M. Rigol, Scaling of the gap, fidelity susceptibility, and Bloch oscillations across the superfluid to Mott insulator transition in the one-dimensional Bose-Hubbard model,

Phys. Rev. A 87, 043606 (2013).

This paper compares three approaches to determine quantum critical points in the one-dimensional Bose-Hubbard model: a scaling ansatz to obtain an accurate finite-size scaling of the excitation gap, a quantum information approach by computing the fidelity susceptibility (1.4), and a non-equilibrium approach in which the amplitude of Bloch oscillations is used to estimate the phase transition point. This approach can be useful for ongoing experiments with ultra cold gases on optical lattices.

The numerical DMRG results for the gap, the fidelity susceptibility and for the Bloch oscillations are all my contribution. The idea of comparing the phase transition points as obtained from the computation of the gap to the ones resulting from the fidelity susceptibility and the amplitude of Bloch oscillations emerged from some of my previous work. The critical analysis of the behavior of the fidelity susceptibility shows that this quantity needs to be taken with care when investigating for quantum critical behavior (probably a more elaborate finite size scaling analysis is needed), while the results for the Bloch oscillations further support previous findings from my contributions [SRM10, SRM23] (see the next chapter) that this approach can provide an estimate for the critical point by performing an out-of-equilibrium experiment. I had a central role in this project in providing the results and pursuing the various approaches discussed there, in the discussions and interpretations and in the writing of the manuscript.

Link to the published version:

http://dx.doi.org/10.1103/PhysRevA.87.043606

Link to the preprint version:

https://arxiv.org/abs/1212.2219 


\section{Chapter 8}

\section{Quantum Simulation of Superconductivity, Quantum Magnetism, and Topological Phases}

The papers in this chapter deal with the possibility to realize superconducting (or, in the context of ultracold atoms, superfluid) and quantum magnetic models on optical lattices using ultracold polar molecules, see Ch. 4. These models can possess topological phases, see [SRM5] further below. The novel aspect of the presented proposal is to use the interactions between the polar molecules to directly emulate such behavior, which leads directly to a generalized t-J-like model without needing to take the strong coupling limit of an underlying Hubbard model (1.5). This allows to realize a wider range of interactions, and also causes additional interactions in the model which can lead to interesting effects. Due to the nature of the dipolar interactions between the molecules, all interactions are long-range, decaying $\sim 1 / r^{3}$ with distance $r$, so that it becomes necessary to test for the relevance of the longrangedness of the interactions for the phase diagrams of the systems.

The first paper and references therein introduces the proposal and presents the ground-state phase diagram for the simplest experimentally realizable case, the $t-J_{\perp}$ chain, and also presents the idea to look for quantum critical behavior in the properties of Bloch oscillations when crossing the phase transition. The second contribution discusses a generalization allowing for interactions which depend on the spatial directions and leading to models which realize symmetry protected topological phases; interestingly, we find 
these phases to survive also in the presence of the long range interactions typical for quantum simulators with ultracold polar molecules. The third paper treats the non-equilibrium dynamics of a simple initial state which helps in verifying the proposal in ongoing experiments, as recently demonstrated in an experiment at JILA [274]. 
A.V. Gorshkov, S.R. Manmana, G. Chen, J. Ye, E. Demler, M.D. Lukin, and A.M. Rey,

Tunable Superfluidity and Quantum Magnetism with Ultracold Polar Molecules, Phys. Rev. Lett. 107, 115301 (2011).

This paper presents a proposal to use recently realized ultracold polar molecules for the emulation of quantum $S=1 / 2$ systems. The idea of the proposal is to use the dipole moment and the resulting interaction between the heteronuclear molecules for the emulation of strongly correlated systems; in particular, by populating only two rotational eigenstates of the molecules, which can be done by forming dressed states induced by external DC electrical fields and microwaves, it is possible to realize two-level systems, which emulate interacting itinerant and static $S=1 / 2$ systems. This encompasses to possibility to emulate superconducting as well as quantum magnetic states of matter. My role in this project was a central one: the publication is based on extensive discussions in the collaboration exploring possibilities for the realization of interesting quantum many body behavior, for identifying the simplest experimentally realizable case, obtaining the phase diagrams using the DMRG in this case, checking for the effect of the long-range interactions by computing the corresponding phase diagrams with and without long-range interactions, and contributing with the idea to use Bloch oscillations in the search for phase transitions in non-equilibrium experiments on optical lattices; correspondingly, my role was central for the writing of the paper, in particular the part involving the numerical results. In December 2011, I was awarded with the DARPA-OLE best paper (theory) award for this publication.

Link to the published version:

http://dx.doi.org/10.1103/PhysRevLett.107.115301

Link to the preprint version:

https://arxiv.org/abs/1106.1644 
S.R. Manmana, E.M. Stoudenmire, K.R.A. Hazzard, A.M. Rey, and A.V. Gorshkov,

Topological phases in ultracold polar-molecule quantum magnets, Phys. Rev. B (rapid communication) 87, 081106(R) (2013).

This paper introduces a setup to realize systems with spatially inhomogeneous interactions in polar molecule systems on optical lattices. This extension of the proposal presented in the previous paper allows for the realization of various models which host topological phases. The paper then treats the effect of long-range interactions on symmetry protected topological (SPT) phases (see Sec. 4.3) in a two-leg ladder system with spatially inhomogeneous interactions (in the present case the interactions along the legs are different from the ones along the rungs). We treat an extension of a model analyzed by X.-G. Wen and collaborators to discuss properties of SPT phases and to distinguish between different SPT phases by considering the behavior of so-called 'active operators' [301]. My contribution to the paper is central: using DMRG and MPS/MPO approaches, in close collaboration with E.M. Stoudenmire I obtained the phase diagram of the system with and without long-range interactions, and I contributed substantially in the writing of the paper and to the underlying discussions. I introduced the quantity which we coined 'entanglement splitting'; this is a single number being zero if the entanglement spectrum is (at least) two-fold degenerate and finite otherwise. This is very useful in identifying transitions from SPT phases to non-topological gapped phases as discussed in the paper, and helped in identifying SPT phases also in the presence of long-range interactions. It would be interesting to do a similar analysis in a system with long-range interactions decaying with arbitrary exponent, $\sim 1 / r^{\alpha}$, and to see if there is a threshold value for $\alpha$ at which the SPT phases cease to exist.

Link to the published version:

http://dx.doi.org/10.1103/PhysRevB.87.081106

Link to the preprint version (including supplementary material):

https://arxiv.org/abs/1210.5518 
K.R.A. Hazzard, S.R. Manmana, M. Foss-Feig, and A.M. Rey, Far from equilibrium quantum magnetism with ultracold polar molecules, Phys. Rev. Lett. 110, 075301 (2013).

This paper takes on the proposal discussed in [SRM10] and addresses the question what can be done in experiments today. This is necessary since the experimental realization of quantum magnetic states is hampered by the difficulty of reaching temperatures well below the Fermi-temperature and entropies low enough for obtaining quantum many body effects. In this proposal, the idea is to consider the time evolution of simple initial states at arbitrary temperatures and look for signatures of quantum magnetism in the subsequent dynamics. A recent experiment performed at JILA [274] takes on the ideas presented in this paper and finds for the first time signatures for the realization of effective spin-interactions in polar molecule systems. My contributions to the paper are central since I contributed all the numerical results presented (in particular also the numerically costly results in the presence of disorder) and I contributed substantially in the underlying discussions which lead to the paper and in its writing.

Link to the published version:

http://dx.doi.org/10.1103/PhysRevLett.110.075301

Link to the preprint version:

https://arxiv.org/abs/1209.4076 


\section{Chapter 9}

\section{Non-Equilibrium Dynamics}

In this chapter, some of my work concerning the non-equilibrium dynamics of strongly correlated quantum systems is presented. The first section deals with so-called quantum quenches, in which the system is pushed outof-equilibrium by suddenly changing one of the parameters. This typically leads to interesting behavior, and here we focus on the propagation of correlations, the formation of quasiparticles and prethermalization behavior, and the emergence of a 'negative temperature state' following a quantum quench.

The second section treats issues related to transport in systems of cold gases on optical lattices, where the notion of transport is taken in a wider sense. This includes the dynamics after releasing particles from a trap, transport when tilting an optical lattice, and properties of solitons in interacting lattice systems. 


\subsection{Quantum Quenches}

S.R. Manmana, S. Wessel, R.M. Noack, and A. Muramatsu,

Time evolution of correlations in strongly interacting fermions after a quantum quench,

Phys. Rev. B 79, 155104 (2009).

This paper discusses the dynamics of correlation functions in a lattice system with short-range interactions following a quantum quench in the interaction strength. Over a wide range of parameters, a light-cone-effect can be identified, which can be understood in terms of ballistically propagating quasiparticles created by the quench, as proposed by Calabrese and Cardy [372], and which conforms with the notion of a Lieb-Robinson bound which gives an upper speed for the propagation of correlations and information in a lattice system. Interestingly, the effect seems to persist also in regions beyond the validity of the conformal field theory treatment of Calabrese and Cardy. On the other hand, for quenches starting from a gapless Luttinger liquid phase deep into the Mott-insulating regime, on the time scales treated, domain walls are created, reminiscent of the Kibble-Zurek mechanism. In the meantime, the light-cone-effect has been observed in experiments on optical lattices [374], and in a recent contribution [SRM2], the question is addressed to which extend a Lieb-Robinson bound (i.e., such a 'causal' propagation of correlations and information) persists in the presence of long-range interactions decaying $\sim 1 / r^{\alpha}$ with distance $r$. It appears that the causal region can become non-linear, and for systems in $D$ spatial dimensions for a generic situation no Lieb-Robinson bound can be identified if $\alpha<D$ for generic initial states and $\alpha<D / 2$ for product initial states. My contributions to this paper are all of the results, and I was centrally involved in the interpretation of the findings and the discussions underlying the publication as well as its writing.

Link to the published version:

http://dx.doi.org/10.1103/PhysRevB.79.155104

Link to the preprint version:

https://arxiv.org/abs/0812.0561 
S. Mandt, A.E. Feiguin, and S.R. Manmana,

Relaxation towards negative temperatures in bosonic systems:

generalized Gibbs ensembles and beyond integrability,

Phys. Rev. A 88, 043643 (2013).

This paper treats a specific quench which leads to a final state which can be associated to 'negative absolute temperatures'. This is a notion well known since the 1950ies from nuclear spin systems and essentially describes a situation in which high energy eigenstates of the system are more likely to be occupied than low energy states. In contrast to systems with inverted energy populations (like lasers), however, the population inversion is stable in time and all laws of thermodynamics apply to the system. In this paper, integrable 'hard-core' bosons are treated, which allowed us to numerically treat the dynamics on very long time scales and in this way to follow the formation of the negative temperature state. We also introduce a notion which defines such a state in the context of generalized Gibbs ensembles for a negative temperature state, see the description in the paper. The formation of the negative temperature state can be understood in terms of a dynamical symmetry of the underlying Hubbard model, and we discuss to which extend it survives when leaving the ideal case of a product initial state. My contribution to this paper lies in guiding the first author in the implementation of the code for treating the systems and in obtaining the numerical results, in the interpretation and underlying discussions of the results in the hard-core limit, in obtaining the exact diagonalization results of Sec. V which present results beyond the integrable 'hard-core' boson case, and in the writing of the paper.

Link to the published version:

http://dx.doi.org/10.1103/PhysRevA.88.043643

Link to the preprint version:

https://arxiv.org/abs/1307.7188 
F.H.L. Essler, S. Kehrein, S.R. Manmana, and N.J. Robinson, Quench dynamics in a model with tuneable integrability breaking, Phys. Rev. B 89, 165104 (2014).

This paper treats the quench in a 1D interacting model in which the degree of breaking the integrability can be tuned. This allows for the systematic investigation of the effect of integrability on the relaxation and thermalization behavior of $1 \mathrm{D}$ strongly correlated quantum systems. In combination of the adaptive t-DMRG and the (semi-) analytical CUTs approach, we identify the creation of quasiparticles due to the quench and the formation of a (possibly) metastable state which corresponds to a prethermalization scenario. Interestingly, despite the tuning of the integrability breaking and the rather large system sizes and relatively long times reached, it is not possible to identify a crossover from the prethermalized state to the expected thermal state. The question arises if this is due to enhanced time scales in $1 \mathrm{D}$, that the breaking of integrability is still not strong enough, or whether there are fundamental issues inhibiting the formation of a thermal state in the configuration studied. My contribution to this paper is central since I provided all the numerical t-DMRG results, I was involved in the discussions and interpretation of the results and in the writing of the paper.

Link to the published version:

http://dx.doi.org/10.1103/PhysRevB.89.165104

Link to the preprint version:

https://arxiv.org/abs/1311.4557 


\subsection{Transport on Optical Lattices}

F. Heidrich-Meisner, S.R. Manmana, M. Rigol, A. Muramatsu, A.E. Feiguin, and E. Dagotto,

Quantum distillation: dynamical generation of low-entropy states of strongly correlated fermions in an optical lattice,

Phys. Rev. A (rapid communication) 80, 041603(R) (2009).

This paper treats interacting fermions when released from an initial confining potential. Naively, one would expect the particles to flow out onto the empty part of the lattice, with a speed depending on the interaction strength. Interestingly, however, repulsive interactions can block the flow into the empty space, and somewhat counterintuitively lead to a pile-up of the particles. This leads to the dynamical generation of a metastable low-entropy state in the originally confined region of the lattice, an effect we coined 'quantum distillation'. My contributions to this paper are time-dependent exact diagonalization as well as time-dependent DMRG results in close collaboration with the other authors, to discussions and interpretations of the results and to the writing of the paper.

Link to the published version:

http://dx.doi.org/10.1103/PhysRevA.80.041603

Link to the preprint version (including supplementary material):

https://arxiv.org/abs/0903.2017 
C.P. Rubbo, S.R. Manmana, B.M. Peden, M.J. Holland, and A.M. Rey, Resonantly Enhanced Tunneling and Transport of Ultracold Atoms on Tilted Optical Lattices,

Phys. Rev. A 84, 033638 (2011).

This paper treats the dynamics of bosons on an optical lattice after tilting the lattice. Interestingly, since the system is a closed one, Bloch oscillations are the dominant effect which inhibits mass transport through the lattice. The question arises, if it is possible to create transport in an optical lattice and overcome the Bloch oscillations. A particular focus is put on the resonant case, in which the interaction strength equals the difference in local chemical potential between two neighboring sites. In this case, two interesting effects are obtained: first, as pointed out by Sachdev et al. [391] it is possible to derive an effective $S=1 / 2$ Ising-like model in a transverse and a longitudinal magnetic field. In the meantime, this proposal has been realized in an experiment in M. Greiner's group at Harvard University [392] which represents one of the first realizations of quantum magnetism on optical lattices. The second interesting aspect which we propose in this work is that by periodically modulating the lattice strength, it is indeed possible to enhance transport, a mechanism which we coined 'slinky scheme'. A third aspect is that we obtain indications that the Bloch oscillations can give a hint for quantum critical behavior, similar to the observations made in my contributions [SRM10, SRM20]. Most of the results were obtained by C.P. Rubbo, who at that time was a PhD student in the group of Prof. A.M. Rey at JILA, and whose co-supervisor I was during the last year of his $\mathrm{PhD}$. The numerical results presented were obtained using my time-dependent DMRG code and under the supervision and in intense collaboration between him, Prof. Rey and myself. I contributed substantially to discussions and the interpretations of the findings and to the writing of the paper.

Link to the published version:

http://dx.doi.org/10.1103/PhysRevA.84.033638

Link to the preprint version:

https://arxiv.org/abs/1106.0326 
C.P. Rubbo, I.I. Satija, W.P. Reinhardt, R. Balakrishnan, A.M. Rey, and S.R. Manmana,

Quantum dynamics of solitons in strongly interacting systems on optical lattices,

Phys. Rev. A 85, 053617 (2012).

This paper tests a proposal of I.I. Satija and collaborators [390] for the creation of 'bright' solitons in cold gases set-ups. In this paper we test for the possibility to realize this proposal via $S=1 / 2$ spin systems on a lattice and for the stability of these soliton-solutions by preparing the according initial states and then performing the time evolution with the corresponding interacting spin model using the adaptive t-DMRG. Indeed, on the time scales accessible to us, the soliton solutions appear to be rather stable in time, so that we expect that in corresponding experiments on optical lattices on the typical time scales of these experiments the effects should become visible. Interestingly, replacing the soliton initial state by a simple Gaussian wave packet leads to a similar stability of the initial state, but only if a discontinuity in the phase of the wave function (as requested by the soliton solution) is present, otherwise the wave packet disperses immediately. This might be helpful for the realization of these effects in experiments since the constraints on the initial state appear to be somewhat relaxed. My contribution to this paper was to work intensely with C.P. Rubbo, who at that time was a $\mathrm{PhD}$ student in the group of Prof. A.M. Rey at JILA and whose co-supervisor I was during the last year of his $\mathrm{PhD}$. The numerical results were obtained by him using my time-dependent DMRG code. I contributed substantially to the discussions and interpretations of the findings as well as to the writing of the paper.

Link to the published version:

http://dx.doi.org/10.1103/PhysRevA.85.053617

Link to the preprint version:

https://arxiv.org/abs/1202.3400 


\section{Acknowledgements}

The research presented in this thesis would not have been possible without the contribution of many people. Most of all, I would like to thank Prof. Thomas Pruschke for giving me the opportunity for pursuing my Habilitation and all the support I am experiencing, despite many difficulties, and the Institute for Theoretical Physics at the University of Göttingen. I also thank Prof. Stefan Kehrein and Prof. Andreas Honecker for their continuous support during this time. The environment at our institute is very lively and friendly, and I appreciate the contributions of all members to the friendly and active atmosphere, and a big thank you goes to everyone working here (allow me not to mention everyone name by name - I am sure I would forget someone which I do not intend to do, and so please accept my collective thanks to everyone). Equally, I want to thank all my collaborators over the past years who helped me realizing interesting research and for the countless exciting and lively discussions (again, allow me not to mention everyone name by name - I am sure I would forget someone which I do not intend to do, and so please accept my collective thanks to everyone), and the hospitality at all places I had a chance to be at and to visit. Last but not least, let me acknowledge the funding I obtained in the past, in particular also the possibility to be principal investigator and for the ongoing support by the SFB/CRC 1073 at the University of Göttingen as well as the Virtual Institute of the Helmholtz foundation 'New States of Matter and their Excitations'. 


\section{Bibliography}

[1] I. Bloch, Nature Physics 1, 23 (2005).

[2] I. Bloch, J. Dalibard, and W. Zwerger, Rev. Mod. Phys. 80, 885 (2008).

[3] J. G. Bednorz and K. A. Müller, Zeitschrift für Physik B Condensed Matter 64, 189 (1986).

[4] E. Dagotto, Rev. Mod. Phys. 66, 763 (1994).

[5] Frustrated Spin Systems, edited by H. T. Diep (World Scientific Publishing, Singapore, 2004).

[6] Quantum Magnetism, Vol. 645 of Lecture Notes in Physics, edited by U. Schollwöck, J. Richter, D. Farnell, and R. Bishop (Springer, Berlin/Heidelberg, 2004).

[7] Introduction to Frustrated Magnetism, Vol. 164 of Springer Series in Solid-State Sciences, edited by C. Lacroix, P. Mendels, and F. Mila (Springer, Berlin / Heidelberg, 2011).

[8] M. Greiner, O. Mandel, T. Esslinger, T. W. Hänsch, and I. Bloch, Nature 415, 39 (2002).

[9] K. Takatsu, W. Shiramura, and H. Tanaka, Journal of the Physical Society of Japan 66, 1611 (1997).

[10] C. Rüegg, N. Cavadini, A. Furrer, H.-U. Güdel, K. Krämer, H. Mutka, A. Wildes, K. Habicht, and P. Vorderwisch, Nature 423, 62 (2003).

[11] H. Kageyama, K. Yoshimura, R. Stern, N. V. Mushnikov, K. Onizuka, M. Kato, K. Kosuge, C. P. Slichter, T. Goto, and Y. Ueda, Phys. Rev. Lett. 82, 3168 (1999).

[12] K. Kodama, M. Takigawa, M. Horvatic, C. Berthier, H. Kageyama, Y. Ueda, S. Miyahara, F. Becca, and F. Mila, Science 298, 395 (2002). 
[13] M. P. Shores, E. A. Nytko, B. M. Bartlett, and D. G. Nocera, Journal of the American Chemical Society 127, 13462 (2005).

[14] M. Matsumoto, B. Normand, T. M. Rice, and M. Sigrist, Phys. Rev. Lett. 89, 077203 (2002).

[15] M. Jaime, V. F. Correa, N. Harrison, C. D. Batista, N. Kawashima, Y. Kazuma, G. A. Jorge, R. Stern, I. Heinmaa, S. A. Zvyagin, Y. Sasago, and K. Uchinokura, Phys. Rev. Lett. 93, 087203 (2004).

[16] T. Giamarchi, C. Rüegg, and O. Tchernyshyov, Nature Physics 4, 198 (2008).

[17] M. Oshikawa, M. Yamanaka, and I. Affleck, Phys. Rev. Lett. 78, 1984 (1997).

[18] T. M. Rice, Science 298, 760 (2002).

[19] T. Momoi and K. Totsuka, Phys. Rev. B 62, 15067 (2000).

[20] K.-K. Ng and T. K. Lee, Phys. Rev. Lett. 97, 127204 (2006).

[21] K. Ng and T. Lee, J. Magn. Mag. Mat. 310, 1371 (2007).

[22] P. Sengupta and C. D. Batista, Phys. Rev. Lett. 98, 227201 (2007).

[23] N. Laflorencie and F. Mila, Phys. Rev. Lett. 99, 027202 (2007).

[24] K. P. Schmidt, J. Dorier, A. M. Läuchli, and F. Mila, Phys. Rev. Lett. 100, 090401 (2008).

[25] O. Penrose and L. Onsager, Phys. Rev. 104, 576 (1956).

[26] E. Kim and M. H. W. Chan, Nature 427, 225 (2004).

[27] E. Kim and M. H. W. Chan, Science 305, 1941 (2004).

[28] S. Sasaki, R. Ishiguro, F. Caupin, H. J. Maris, and S. Balibar, Science 313, 1098 (2006).

[29] N. Prokof'ev, Advances in Physics 56, 381 (2007).

[30] S. Sachdev, Quantum Phase Transitions (Cambridge University Press, Cambridge, 1999).

[31] M. Vojta, Reports on Progress in Physics 66, 2069 (2003).

[32] S.-A. Cheong and C. L. Henley, Phys. Rev. B 79, 212402 (2009).

[33] S.-A. Cheong and C. L. Henley, Phys. Rev. B 80, 165124 (2009). 
[34] W. Münder, A. Weichselbaum, A. Holzner, J. von Delft, and C. L. Henley, New Journal of Physics 12, 075027 (2010).

[35] M. Henkel, Conformal invariance and critical phenomena (Springer, Berlin, 1999).

[36] A. M. Tsvelik, Quantum Field Theory in Condensed Matter Physics (Cambridge University Press, Cambridge, 2003).

[37] G. Vidal, J. I. Latorre, E. Rico, and A. Kitaev, Phys. Rev. Lett. 90, $227902(2003)$.

[38] P. Calabrese and J. Cardy, J. Stat. Mech.: Theor. and Exp. P06002 (2004).

[39] S. R. White, Phys. Rev. Lett. 69, 2863 (1992).

[40] S. R. White, Phys. Rev. B 48, 10345 (1993).

[41] M. Nielsen and I. Chuang, Quantum Computation and Quantum Information (Cambridge University Press, Cambridge, 2000).

[42] P. Zanardi and N. Paunković, Phys. Rev. E 74, 031123 (2006).

[43] M. Cozzini, P. Giorda, and P. Zanardi, Phys. Rev. B 75, 014439 (2007).

[44] L. Campos Venuti and P. Zanardi, Phys. Rev. Lett. 99, 095701 (2007).

[45] L. Campos Venuti, M. Cozzini, P. Buonsante, F. Massel, N. Bray-Ali, and P. Zanardi, Phys. Rev. B 78, 115410 (2008).

[46] S.-J. Gu, H.-M. Kwok, W.-Q. Ning, and H.-Q. Lin, Phys. Rev. B 77, 245109 (2008).

[47] M. Rigol, B. S. Shastry, and S. Haas, Phys. Rev. B 80, 94529 (2009).

[48] A. F. Albuquerque, F. Alet, C. Sire, and S. Capponi, Phys. Rev. B 81, 64418 (2010).

[49] C. N. Varney, K. Sun, V. Galitski, and M. Rigol, Phys. Rev. Lett. 107, 77201 (2011).

[50] J. Sirker, Phys. Rev. Lett. 105, 117203 (2010).

[51] M. J. Stephen and J. P. Straley, Rev. Mod. Phys. 46, 617 (1974).

[52] M. Blume and Y. Y. Hsieh, Journal of Applied Physics 40, 1249 (1969).

[53] H. H. Chen and P. M. Levy, Phys. Rev. Lett. 27, 1383 (1971).

[54] A. F. Andreev and I. A. Grishchuk, Sov. Phys. JETP 60, 267 (1984). 
[55] N. Papanicolaou, Nuclear Physics B 305, 367 (1988).

[56] A. Chubukov, J. Phys. Condens. Matter 2, 1593 (1990).

[57] P. Chandra and P. Coleman, Phys. Rev. Lett. 66, 100 (1991).

[58] V. Barzykin, L. P. Gor'kov, and A. V. Sokol, EPL (Europhysics Letters) 15, 869 (1991).

[59] V. Barzykin and L. P. Gor'kov, Phys. Rev. Lett. 70, 2479 (1993).

[60] A. Smerald and N. Shannon, Phys. Rev. B 88, 184430 (2013).

[61] A. Läuchli, G. Schmid, and S. Trebst, Phys. Rev. B 74, 144426 (2006).

[62] P. Corboz, A. M. Läuchli, K. Totsuka, and H. Tsunetsugu, Phys. Rev. B 76, 220404 (2007).

[63] A. Läuchli, F. Mila, and K. Penc, Phys. Rev. Lett. 97, 87205 (2006).

[64] H. Tsunetsugu and M. Arikawa, Journal of the Physical Society of Japan 75, 083701 (2006).

[65] S. Nakatsuji, Y. Nambu, H. Tonomura, O. Sakai, S. Jonas, C. Broholm, H. Tsunetsugu, Y. Qiu, and Y. Maeno, Science 309, 1697 (2005).

[66] A. V. Gorshkov, M. Hermele, V. Gurarie, C. Xu, P. S. Julienne, J. Ye, P. Zoller, E. Demler, M. D. Lukin, and A. M. Rey, Nature Physics 6, 289 (2010).

[67] C. Nayak, S. H. Simon, A. Stern, M. Freedman, and S. D. Sarma, Rev. Mod. Phys. 80, 1083 (2008).

[68] L. Balents, Nature 464, 199 (2010).

[69] S. Yan, D. A. Huse, and S. R. White, Science 332, 1173 (2011).

[70] S. Depenbrock, I. Mcculloch, and U. Schollwöck, Phys. Rev. Lett. 109, 067201 (2012).

[71] H.-C. Jiang, Z. Wang, and L. Balents, Nature Physics 8, 902 (2012).

[72] P. Lee, Science 321, 1306 (2008).

[73] J. Hubbard, Proc. R. Soc. (London) A 276, 238 (1963).

[74] M. C. Gutzwiller, Phys. Rev. Lett. 10, 159 (1963).

[75] J. Kanamori, Prog. Theor. Phys. 30, 275 (1963). 
[76] F. H. L. Essler, H. Frahm, F. Göhmann, A. Klümper, and V. E. Korepin, The One-Dimensional Hubbard Model (Cambridge University Press, Cambridge, 2005).

[77] M. P. A. Fisher, P. B. Weichman, G. Grinstein, and D. S. Fisher, Phys. Rev. B 40, 546 (1989).

[78] Y. I. Manin, Computable and uncomputable (in Russian); Moscow, Sovetskoye Radio 1980. See appendix of arXiv:quant-ph/9903008 for an english translation.

[79] R. P. Feynman, Int. J. Theor. Phys. 21, 467 (1982).

[80] R. P. Feynman, Found. Phys. 16, 507 (1985).

[81] R. P. Feynman, Optics News 11, 11 (February 1985).

[82] A. Auerbach, Interacting Electrons and Quantum Magnetism (Springer, New York, 1994).

[83] F. C. Zhang and T. M. Rice, Phys. Rev. B 37, 3759 (1998).

[84] I. Affleck and J. B. Marston, Phys. Rev. B 37, 3774 (1988).

[85] R. Assaraf, P. Azaria, M. Caffarel, and P. Lecheminant, Phys. Rev. B 60, 2299 (1999).

[86] C. Wu, J.-P. Hu, and S.-C. Zhang, Phys. Rev. Lett. 91, 186402 (2003).

[87] R. Assaraf, P. Azaria, E. Boulat, M. Caffarel, and P. Lecheminant, Phys. Rev. Lett. 93, 016407 (2004).

[88] C. Honerkamp and W. Hofstetter, Phys. Rev. Lett. 92, 170403 (2004).

[89] P. Lecheminant, E. Boulat, and P. Azaria, Phys. Rev. Lett. 95, 240402 (2005).

[90] E. Szirmai and J. Sólyom, Phys. Rev. B 71, 205108 (2005).

[91] F. F. Assaad, Phys. Rev. B 71, 075103 (2005).

[92] C. Wu, Phys. Rev. Lett. 95, 266404 (2005).

[93] E. Szirmai and J. Sólyom, Phys. Rev. B 74, 155110 (2006).

[94] Á. Rapp, G. Zaránd, C. Honerkamp, and W. Hofstetter, Phys. Rev. Lett. 98, 160405 (2007).

[95] J. Zhao, K. Ueda, and X. Wang, J. Phys. Soc. Jpn. 76, 4711 (2007). 
[96] K. Buchta, Ö. Legeza, E. Szirmai, and J. Sólyom, Phys. Rev. B 75, 155108 (2007).

[97] P. Lecheminant, P. Azaria, and E. Boulat, Nuclear Physics B 798, 443 (2008).

[98] Á. Rapp, W. Hofstetter, and G. Zaránd, Phys. Rev. B 77, 144520 (2008).

[99] E. Szirmai, Ö. Legeza, and J. Sólyom, Phys. Rev. B 77, 045106 (2008).

[100] S. Capponi, G. Roux, P. Lecheminant, P. Azaria, E. Boulat, and S. R. White, Phys. Rev. A 77, 13624 (2008).

[101] R. A. Molina, J. Dukelsky, and P. Schmitteckert, Phys. Rev. A 80, 013616 (2009).

[102] E. V. Gorelik and N. Blümer, Phys. Rev. A 80, 51602 (2009).

[103] G. Roux, S. Capponi, P. Lecheminant, and P. Azaria, The European Physical Journal B 68, 293 (2009).

[104] P. Azaria, S. Capponi, and P. Lecheminant, Phys. Rev. A 80, 41604 (2009).

[105] G. Klingschat and C. Honerkamp, Phys. Rev. B 82, 94521 (2010).

[106] T. Ulbricht, R. A. Molina, R. Thomale, and P. Schmitteckert, Phys. Rev. A 82, 011603 (2010).

[107] E. Szirmai and M. Lewenstein, Europhysics Lett. 93, 66005 (2011).

[108] H. Nonne, P. Lecheminant, S. Capponi, G. Roux, and E. Boulat, Phys. Rev. B 81, 20408 (2010).

[109] K. R. A. Hazzard, V. Gurarie, M. Hermele, and A. M. Rey, Phys. Rev. A 85, 041604 (2012).

[110] B. Sutherland, Phys. Rev. B 12, 3795 (1975).

[111] N. Read and S. Sachdev, Phys. Rev. Lett. 62, 1694 (1989).

[112] I. Affleck, D. P. Arovas, J. B. Marston, and D. A. Rabson, Nuclear Physics B 366, 467 (1991).

[113] K. Harada, N. Kawashima, and M. Troyer, Phys. Rev. Lett. 90, 117203 (2003).

[114] P. Li and S.-Q. Shen, New Journal of Physics 6, 160 (2004). 
[115] A. Paramekanti and J. B. Marston, Journal of Physics: Condensed Matter 19, 5215 (2007).

[116] M. Führinger, S. Rachel, R. Thomale, M. Greiter, and P. Schmitteckert, Annalen der Physik 17, 922 (2008).

[117] A. Kolezhuk, Phys. Rev. B 78, 144428 (2008).

[118] M. Aguado, M. Asorey, E. Ercolessi, F. Ortolani, and S. Pasini, Phys. Rev. B 79, 12408 (2009).

[119] N. Kawashima and Y. Tanabe, Phys. Rev. Lett. 98, 57202 (2007).

[120] R. Thomale, D. Schuricht, and M. Greiter, Phys. Rev. B 75, 24405 (2007).

[121] C. Xu and C. Wu, Phys. Rev. B 77, 134449 (2008).

[122] S. Rachel, R. Thomale, M. Führinger, P. Schmitteckert, and M. Greiter, Phys. Rev. B 80, 180420 (2009).

[123] S. Rachel, D. Schuricht, B. Scharfenberger, R. Thomale, and M. Greiter, Journal of Physics: Conference Series 200, 022049 (2010).

[124] K. S. D. Beach, F. Alet, M. Mambrini, and S. Capponi, Phys. Rev. B 80, 184401 (2009).

[125] P. Li, G.-M. Zhang, and S.-Q. Shen, Phys. Rev. B 75, 104420 (2007).

[126] T. A. Tóth, A. M. Läuchli, F. Mila, and K. Penc, Phys. Rev. Lett. 105, 265301 (2010).

[127] I. Affleck, T. Kennedy, E. H. Lieb, and H. Tasaki, Phys. Rev. Lett. 59, 799 (1987).

[128] I. Affleck, T. Kennedy, E. H. Lieb, and H. Tasaki, Communications in Mathematical Physics 115, 477 (1988).

[129] M. Greiter and S. Rachel, Phys. Rev. B 75, 184441 (2007).

[130] M. Greiter, S. Rachel, and D. Schuricht, Phys. Rev. B 75, 60401 (2007).

[131] S. Rachel and M. Greiter, Phys. Rev. B 78, 134415 (2008).

[132] M. Hermele, V. Gurarie, and A. M. Rey, Phys. Rev. Lett. 103, 135301 (2009).

[133] F. Alet, S. Capponi, H. Nonne, P. Lecheminant, and I. P. McCulloch, Phys. Rev. B 83, 60407 (2011). 
[134] J. Lou, A. W. Sandvik, and N. Kawashima, Phys. Rev. B 80, 180414 (2009).

[135] V. Kalmeyer and R. B. Laughlin, Phys. Rev. Lett. 59, 2095 (1987).

[136] X. G. Wen, F. Wilczek, and A. Zee, Phys. Rev. B 39, 11413 (1989).

[137] A. Kitaev, Annals of Physics 321, 2 (2006).

[138] U. Schollwöck, Annals of Physics 326, 96 (2011).

[139] U. Schollwöck, Rev. Mod. Phys. 77, 259 (2005).

[140] Density Matrix Renormalization - A New Numerical Method in Physics, edited by I. Peschel, X. Wang, M. Kaulke, and K. Hallberg (Springer Verlag, Berlin, 1999).

[141] K. Hallberg, Adv. Phys. 55, 477 (2006).

[142] A. L. Malvezzi, Braz. Journ. of Phys. 33, 55 (March 2003).

[143] http://quattro.phys.sci.kobe-u.ac.jp/dmrg/condmat.html, maintained by T. Nishino, updated daily.

[144] M. B. Hastings, Journal of Statistical Mechanics: Theory and Experiment 2007, P08024 (2007).

[145] J. Eisert, M. Cramer, and M. B. Plenio, Rev. Mod. Phys. 82, 277 (2010).

[146] S. R. White and A. L. Chernyshev, Phys. Rev. Lett. 99, 127004 (2007).

[147] Z. Zhu, D. Huse, and S. White, Phys. Rev. Lett. 111, 257201 (2013).

[148] E. M. Stoudenmire and S. R. White, Annual Review of Condensed Matter Physics 3, 111 (2012).

[149] E. M. Stoudenmire and S. R. White, Phys. Rev. B 87, 155137 (2013).

[150] O. Legeza and J. Sólyom, Phys. Rev. B 68, 195116 (2003).

[151] J. Rissler, R. M. Noack, and S. R. White, Chemical Physics 323, 519 (2006).

[152] M. Fannes, B. Nachtergaele, and R. Werner, Communications in Mathematical Physics 144, 443 (1992).

[153] S. Östlund and S. Rommer, Phys. Rev. Lett. 75, 3537 (1995).

[154] I. P. McCulloch, Journal of Statistical Mechanics: Theory and Experiment 2007, P10014 (2007). 
[155] F. Verstraete, V. Murg, and J. I. Cirac, Advances in Physics 57, 143 (2008).

[156] J. I. Cirac and F. Verstraete, Journal of Physics A: Mathematical and Theoretical 42, 504004 (2009).

[157] F. Verstraete, D. Porras, and J. I. Cirac, Phys. Rev. Lett. 93, 227205 (2004).

[158] P. Pippan, S. R. White, and H. G. Evertz, Phys. Rev. B 81, 081103 (2010).

[159] F. Verstraete and J. I. Cirac, cond-mat/0407066 (unpublished).

[160] G. Sierra and M. Martin-Delgado, arXiv:cond-mat/9811170v3 (1998).

[161] J. Jordan, R. Orús, G. Vidal, F. Verstraete, and J. I. Cirac, Phys. Rev. Lett. 101, 250602 (2008).

[162] P. Corboz, R. Orús, B. Bauer, and G. Vidal, Phys. Rev. B 81, 165104 (2010).

[163] G. Vidal, Phys. Rev. Lett. 99, 220405 (2007).

[164] G. Vidal, Phys. Rev. Lett. 101, 110501 (2008).

[165] V. Giovannetti, S. Montangero, and R. Fazio, Phys. Rev. Lett. 101, 180503 (2008).

[166] P. Corboz and G. Vidal, Phys. Rev. B 80, 165129 (2009).

[167] V. Murg, F. Verstraete, O. Legeza, and R. M. Noack, Phys. Rev. B 82, 205105 (2010).

[168] B. Bauer, G. Vidal, and M. Troyer, Journal of Statistical Mechanics: Theory and Experiment 2009, P09006 (2009).

[169] P. Corboz, J. Jordan, and G. Vidal, Phys. Rev. B 82, 245119 (2010).

[170] P. Corboz, S. R. White, G. Vidal, and M. Troyer, Phys. Rev. B 84, 041108 (2011).

[171] D. Poilblanc, P. Corboz, N. Schuch, and J. I. Cirac, Phys. Rev. B 89, 241106 (2014).

[172] P. Corboz and F. Mila, Phys. Rev. B 87, 115144 (2013).

[173] P. Corboz and F. Mila, Phys. Rev. Lett. 112, 147203 (2014). 
[174] B. Bauer, P. Corboz, A. M. Läuchli, L. Messio, K. Penc, M. Troyer, and F. Mila, Phys. Rev. B 85, 125116 (2012).

[175] P. Corboz, K. Penc, F. Mila, and A. M. Läuchli, Phys. Rev. B 86, 041106 (2012).

[176] P. Corboz, M. Lajkó, K. Penc, F. Mila, and A. M. Läuchli, Phys. Rev. B 87, 195113 (2013).

[177] G. Evenbly and G. Vidal, Phys. Rev. Lett. 104, 187203 (2010).

[178] S. R. White, Phys. Rev. Lett. 102, 190601 (2009).

[179] E. M. Stoudenmire and S. R. White, New Journal of Physics 12, 055026 (2010).

[180] U. Schollwöck, Physics 2, 39 (2009).

[181] A. W. Sandvik and G. Vidal, Phys. Rev. Lett. 99, 220602 (2007).

[182] N. Schuch, M. M. Wolf, F. Verstraete, and J. I. Cirac, Phys. Rev. Lett. 100, 040501 (2008).

[183] L. Amico, R. Fazio, A. Osterloh, and V. Vedral, Rev. Mod. Phys. 80, 517 (2008).

[184] E. Fradkin and J. E. Moore, Phys. Rev. Lett. 97, 050404 (2006).

[185] M. Srednicki, Phys. Rev. Lett. 71, 666 (1993).

[186] G. M. Crosswhite, A. C. Doherty, and G. Vidal, Phys. Rev. B 78, 035116 (2008).

[187] B. Pirvu, V. Murg, J. I. Cirac, and F. Verstraete, New Journal of Physics 12, 025012 (2010).

[188] M. B. Hastings, Phys. Rev. B 76, 035114 (2007).

[189] A. J. Daley, C. Kollath, U. Schollwöck, and G. Vidal, J. Stat. Mech.: Theor. Exp. P04005 (2004).

[190] G. Vidal, Phys. Rev. Lett. 91, 147902 (2003).

[191] G. Vidal, Phys. Rev. Lett. 93, 040502 (2004).

[192] S. R. White and A. E. Feiguin, Phys. Rev. Lett. 93, 076401 (2004).

[193] P. Schmitteckert, Phys. Rev. B 70, 121302(R) (2004).

[194] U. Schollwöck, J. Phys. Soc. Jpn. (Suppl.) 74, 246 (2005). 
[195] D. Gobert, C. Kollath, U. Schollwöck, and G. Schütz, Phys. Rev. E 71, 036102 (2005).

[196] A. E. Feiguin and S. R. White, Phys. Rev. B 72, 020404(R) (2005).

[197] F. Verstraete, J. J. Garcia-Ripoll, and J. I. Cirac, Phys. Rev. Lett. 93, 207204 (2004).

[198] M. Zwolak and G. Vidal, Phys. Rev. Lett. 93, 207205 (2004).

[199] I. Pižorn, V. Eisler, S. Andergassen, and M. Troyer, New Journal of Physics 16, 073007 (2014).

[200] T. Barthel, U. Schollwöck, and S. R. White, Phys. Rev. B 79, 245101 (2009).

[201] S. R. White and I. Affleck, Phys. Rev. B 77, 134437 (2008).

[202] R. G. Pereira, S. R. White, and I. Affleck, Phys. Rev. B 79, 165113 (2009).

[203] C. Rüegg, K. Kiefer, B. Thielemann, D. F. McMorrow, V. Zapf, B. Normand, M. B. Zvonarev, P. Bouillot, C. Kollath, T. Giamarchi, S. Capponi, D. Poilblanc, D. Biner, and K. W. Krämer, Phys. Rev. Lett. 101, 247202 (2008).

[204] J. Jaklič and P. Prelovšek, Phys. Rev. B 49, 5065 (1994).

[205] J. Jaklič and P. Prelovšek, Adv. in Phys. 49, 1 (2000).

[206] J. Kokalj and P. Prelovšek, Phys. Rev. B 80, 205117 (2009).

[207] C. Karrasch, J. H. Bardarson, and J. E. Moore, Phys. Rev. Lett. 108, 227206 (2012)

[208] E. Jeckelmann, Phys. Rev. B 66, 045114 (2002).

[209] H. Benthien, F. Gebhard, and E. Jeckelmann, Phys. Rev. Lett. 92, 256401 (2004).

[210] E. Jeckelmann, Progress of Theoretical Physics Supplement 176, 143 (2008).

[211] M. J. Hartmann, J. Prior, S. R. Clark, and M. B. Plenio, Phys. Rev. Lett. 102, 057202 (2009).

[212] S. R. Clark, J. Prior, M. J. Hartmann, D. Jaksch, and M. B. Plenio, New Journal of Physics 12, 025005 (2010).

[213] H. Pichler, A. J. Daley, and P. Zoller, Phys. Rev. A 82, 063605 (2010). 
[214] P. Barmettler, J.-S. Bernier, and C. Kollath, Physics 3, 102 (2010).

[215] N. D. Mermin and H. Wagner, Phys. Rev. Lett. 17, 1133 (1966).

[216] N. D. Mermin and H. Wagner, Phys. Rev. Lett. 17, 1307 (1966).

[217] P. C. Hohenberg, Phys. Rev. 158, 383 (1967).

[218] I. Affleck, Phys. Rev. B 43, 3215 (1991).

[219] B. S. Shastry and B. Sutherland, Physica B+C 108, 1069 (1981).

[220] S. Kehrein, The Flow Equation Approach to Many-Particle Systems, Vol. 217 of Springer Tracts in Modern Physics (Springer, Berlin/Heidelberg, 2006).

[221] K. Penc, J.-B. Fouet, S. Miyahara, O. Tchernyshyov, and F. Mila, Phys. Rev. Lett. 99, 117201 (2007).

[222] I. Dzyaloshinskii, J. Phys. Chem. Solids 4, 241 (1958).

[223] T. Moriya, Phys. Rev. Lett. 4, 228 (1960).

[224] A. Olariu, P. Mendels, F. Bert, F. Duc, J. C. Trombe, M. A. de Vries, and A. Harrison, Phys. Rev. Lett. 100, 087202 (2008).

[225] A. Zorko, S. Nellutla, J. van Tol, L. C. Brunel, F. Bert, F. Duc, J.-C. Trombe, M. A. de Vries, A. Harrison, and P. Mendels, Phys. Rev. Lett. 101, 026405 (2008).

[226] R. Feyerherm, S. Abens, D. Günther, T. Ishida, M. Meißner, M. Meschke, T. Nogami, and M. Steiner, J. Phys.: Condens. Matter 12, 8495 (2000).

[227] S. A. Zvyagin, A. K. Kolezhuk, J. Krzystek, and R. Feyerherm, Phys. Rev. Lett. 93, 027201 (2004).

[228] F. H. L. Eßler, Phys. Rev. B 59, 14376 (1999).

[229] M. Oshikawa and I. Affleck, Phys. Rev. Lett. 79, 2883 (1997).

[230] I. Affleck and M. Oshikawa, Phys. Rev. B 60, 1038 (1999).

[231] I. Affleck and M. Oshikawa, Phys. Rev. B 62, 9200 (2000).

[232] O. Cépas, K. Kakurai, L. P. Regnault, T. Ziman, J. P. Boucher, N. Aso, M. Nishi, H. Kageyama, and Y. Ueda, Phys. Rev. Lett. 87, 167205 (2001). 
[233] Y. F. Cheng, O. Cépas, P. W. Leung, and T. Ziman, Phys. Rev. B 75, $144422(2007)$

[234] V. V. Mazurenko, S. L. Skornyakov, V. I. Anisimov, and F. Mila, Phys. Rev. B 78, 195110 (2008).

[235] J. Romhányi, K. Totsuka, and K. Penc, Phys. Rev. B 83, 024413 (2011).

[236] M. Takigawa, T. Waki, M. Horvatić, and C. Berthier, Journal of the Physical Society of Japan 79, 011005 (2010).

[237] M. Nemec, G. R. Foltin, and K. P. Schmidt, Phys. Rev. B 86, 174425 (2012).

[238] W. Hofstetter, Philosophical Magazine 86, 1891 (2006).

[239] T. Esslinger, Annual Review of Condensed Matter Physics 1, 129 (2010).

[240] S. Fuchs, E. Gull, L. Pollet, E. Burovski, E. Kozik, T. Pruschke, and M. Troyer, Phys. Rev. Lett. 106, 30401 (2011).

[241] S. Trotzky, L. Pollet, F. Gerbier, U. Schnorrberger, I. Bloch, N. V. Prokof'Ev, B. Svistunov, and M. Troyer, Nature Physics 6, 998 (2010).

[242] S. Taie, Y. Takasu, S. Sugawa, R. Yamazaki, T. Tsujimoto, R. Murakami, and Y. Takahashi, Phys. Rev. Lett. 105, 190401 (2010).

[243] C. Wu, Physics 3, 92 (2010).

[244] S. Taie, R. Yamazaki, S. Sugawa, and Y. Takahashi, Nature Physics 8, 825 (2012).

[245] S. Stellmer, R. Grimm, and F. Schreck, Phys. Rev. A 87, 013611 (2013).

[246] G. Pagano, M. Mancini, G. Cappellini, P. Lombardi, F. Schäfer, H. $\mathrm{Hu}$, X.-J. Liu, J. Catani, C. Sias, M. Inguscio, and L. Fallani, Nature Physics 10, 198 (2014).

[247] M. Cazalilla and A. Rey, arXiv:1403.2792 (2014).

[248] R. C. Richardson, Rev. Mod. Phys. 69, 683 (1997).

[249] L. Messio and F. Mila, Phys. Rev. Lett. 109, 205306 (2012).

[250] Z. Cai, H.-h. Hung, L. Wang, D. Zheng, and C. Wu, Phys. Rev. Lett. 110, 220401 (2013). 
[251] S. Capponi and F. F. Assaad, Phys. Rev. B 75, 045115 (2007).

[252] E. Fradkin and F. A. Schaposnik, Phys. Rev. Lett. 66, 276 (1991).

[253] D. F. Schroeter, E. Kapit, R. Thomale, and M. Greiter, Phys. Rev. Lett. 99, 097202 (2007).

[254] B. J. Bloom, T. L. Nicholson, J. R. Williams, S. L. Campbell, M. Bishof, X. Zhang, W. Zhang, S. L. Bromley, and J. Ye, Nature 506, 71 (2014).

[255] A. N. Nikolov, J. R. Ensher, E. E. Eyler, H. Wang, W. C. Stwalley, and P. L. Gould, Phys. Rev. Lett. 84, 246 (2000).

[256] J. M. Sage, S. Sainis, T. Bergeman, and D. DeMille, Phys. Rev. Lett. 94, 203001 (2005).

[257] K.-K. Ni, S. Ospelkaus, M. H. G. de Miranda, A. Pe'er, B. Neyenhuis, J. J. Zirbel, S. Kotochigova, P. S. Julienne, D. S. Jin, and J. Ye, Science 322, 231 (2008).

[258] J. Deiglmayr, A. Grochola, M. Repp, K. Mörtlbauer, C. Glück, J. Lange, O. Dulieu, R. Wester, and M. Weidemüller, Phys. Rev. Lett. 101, 133004 (2008).

[259] F. Lang, K. Winkler, C. Strauss, R. Grimm, and J. H. Denschlag, Phys. Rev. Lett. 101, 133005 (2008).

[260] K. K. Ni, S. Ospelkaus, D. Wang, G. Quemener, B. Neyenhuis, M. H. G. de Miranda, J. L. Bohn, J. Ye, and D. S. Jin, Nature 464, 1324 (2010).

[261] S. Ospelkaus, K.-K. Ni, D. Wang, M. H. G. de Miranda, B. Neyenhuis, G. Quéméner, P. S. Julienne, J. L. Bohn, D. S. Jin, and J. Ye, Science 327, 853 (2010).

[262] S. Ospelkaus, K.-K. Ni, G. Quéméner, B. Neyenhuis, D. Wang, M. H. G. de Miranda, J. L. Bohn, J. Ye, and D. S. Jin, Phys. Rev. Lett. 104, 030402 (2010).

[263] M. H. G. de Miranda, A. Chotia, B. Neyenhuis, D. Wang, G. Quéméner, S. Ospelkaus, J. L. Bohn, J. Ye, and D. S. Jin, Nature Physics 7, 502 (2011).

[264] A. Micheli, G. K. Brennen, and P. Zoller, Nature Physics 2, 341 (2006).

[265] G. K. Brennen, A. Micheli, and P. Zoller, New Journal of Physics 9, 138 (2007). 
[266] G. Pupillo, A. Micheli, H. P. Büchler, and P. Zoller, in Cold Molecules: Theory, Experiment, Applications, edited by R. Krems, W. Stwallye, and B. Friedrich (CRC Press, Boca Raton, FL, 2009).

[267] L. D. Carr and J. Ye, New Journal of Physics 11, 055009 (2009).

[268] L. D. Carr, D. DeMille, R. V. Krems, and J. Ye, New Journal of Physics 11, 055049 (2009).

[269] S. Cornish, Physics 1, 24 (2008).

[270] E. Kuznetsova, M. Gacesa, P. Pellegrini, S. F. Yelin, and R. Côté, New Journal of Physics 11, 055028 (2009).

[271] A. Chotia, B. Neyenhuis, S. A. Moses, B. Yan, J. P. Covey, M. FossFeig, A. M. Rey, D. S. Jin, and J. Ye, Phys. Rev. Lett. 108, 080405 (2012).

[272] M. L. Wall, K. R. A. Hazzard, and A. M. Rey, arXiv:1406.4758 (2014).

[273] B. Zhu, B. Gadway, M. Foss-Feig, J. Schachenmayer, M. L. Wall, K. R. A. Hazzard, B. Yan, S. A. Moses, J. P. Covey, D. S. Jin, J. Ye, M. Holland, and A. M. Rey, Phys. Rev. Lett. 112, 070404 (2014).

[274] B. Yan, S. A. Moses, B. Gadway, J. P. Covey, K. R. A. Hazzard, A. M. Rey, D. S. Jin, and J. Ye, Nature 501, 521 (2013).

[275] R. Z. Bariev, Phys. Rev. B 49, 1474 (1994).

[276] R. Z. Bariev, A. Klümper, A. Schadschneider, and J. Zittartz, Zeitschrift für Physik B Condensed Matter 96, 395 (1995).

[277] Y. Saiga, Y. Kato, and Y. Kuramoto, Journal of the Physical Society of Japan 65, 2361 (1996).

[278] R. Sato, Journal of Physics: Condensed Matter 8, 8363 (1996).

[279] J. Shiraishi, Y. Morita, and M. Kohmoto, Journal of Physics A: Mathematical and General 30, 831 (1997).

[280] T. Fujii, Y. Tsukamoto, and N. Kawakami, Journal of the Physical Society of Japan 66, 2552 (1997).

[281] R.-H. Yue and P. Schlottmann, Phys. Rev. B 66, 85114 (2002).

[282] S. Trotzky, P. Cheinet, S. Folling, M. Feld, U. Schnorrberger, A. M. Rey, A. Polkovnikov, E. A. Demler, M. D. Lukin, and I. Bloch, Science 319, 295 (2008). 
[283] N. Goldenfeld, Lectures on Phase Transitions and the Renormalization Group, Vol. 85 of Frontiers in physics (Addison-Wesley, Advanced Book Program, Boston, 1992).

[284] K. v. Klitzing, G. Dorda, and M. Pepper, Phys. Rev. Lett. 45, 494 (1980).

[285] D. C. Tsui, H. L. Stormer, and A. C. Gossard, Phys. Rev. Lett. 48, 1559 (1982).

[286] R. B. Laughlin, Phys. Rev. Lett. 50, 1395 (1983).

[287] X. G. Wen, Phys. Rev. B 40, 7387 (1989).

[288] X. G. Wen, International Journal of Modern Physics B 04, 239 (1990).

[289] E. Witten, Communications in Mathematical Physics 117, 353 (1988).

[290] X. Chen, Z.-C. Gu, and X.-G. Wen, Phys. Rev. B 83, 035107 (2011).

[291] X. Chen, Z.-C. Gu, and X.-G. Wen, Phys. Rev. B 82, 155138 (2010).

[292] M. Levin and X.-G. Wen, Phys. Rev. Lett. 96, 110405 (2006).

[293] A. M. Turner, F. Pollmann, and E. Berg, Phys. Rev. B 83, 075102 (2011).

[294] Z.-C. Gu and X.-G. Wen, Phys. Rev. B 80, 155131 (2009).

[295] S. Sachdev, Physics 2, 90 (2009).

[296] V. Gurarie, Phys. Rev. B 83, 085426 (2011).

[297] L. Fidkowski and A. Kitaev, Phys. Rev. B 83, 075103 (2011).

[298] H. Li and F. D. M. Haldane, Phys. Rev. Lett. 101, 010504 (2008).

[299] F. Pollmann, A. M. Turner, E. Berg, and M. Oshikawa, Phys. Rev. B 81, 064439 (2010).

[300] F. Pollmann and A. M. Turner, Phys. Rev. B 86, 125441 (2012).

[301] Z.-X. Liu, Z.-B. Yang, Y.-J. Han, W. Yi, and X.-G. Wen, Phys. Rev. B 86, 195122 (2012).

[302] Z.-X. Liu, M. Liu, and X.-G. Wen, Phys. Rev. B 84, 075135 (2011).

[303] Z.-X. Liu, X. Chen, and X.-G. Wen, Phys. Rev. B 84, 195145 (2011).

[304] M. Greiner, O. Mandel, T. W. Hänsch, and I. Bloch, Nature 419, 51 (2002). 
[305] F. B. Anders and A. Schiller, Phys. Rev. Lett. 95, 196801 (2005).

[306] T. Kinoshita, T. Wenger, and D. S. Weiss, Nature 440, 900 (2006).

[307] I. E. Mazets and J. Schmiedmayer, New Journal of Physics 12, 055023 (2010).

[308] M. A. Cazalilla and M. Rigol, New Journal of Physics 12, 055006 (2010).

[309] M. Rigol and A. Muramatsu, Phys. Rev. Lett. 93, 230404 (2004).

[310] F. Heidrich-Meisner, M. Rigol, A. Muramatsu, A. E. Feiguin, and E. Dagotto, Phys. Rev. A 78, 013620 (2008).

[311] M. Rigol and A. Muramatsu, Phys. Rev. Lett. 94, 240403 (2005).

[312] U. Schneider, L. Hackermüller, J. P. Ronzheimer, S. Will, S. Braun, T. Best, I. Bloch, E. Demler, S. Mandt, D. Rasch, and A. Rosch, Nature Physics 8, 213 (2012).

[313] J. P. Ronzheimer, M. Schreiber, S. Braun, S. S. Hodgman, S. Langer, I. P. McCulloch, F. Heidrich-Meisner, I. Bloch, and U. Schneider, Phys. Rev. Lett. 110, 205301 (2013).

[314] S. Langer, F. Heidrich-Meisner, J. Gemmer, I. P. McCulloch, and U. Schollwöck, Phys. Rev. B 79, 214409 (2009).

[315] Y. V. Nazarov and Y. M. Blanter, Quantum Transport: Introduction to Nanoscience (Cambridge University Press, Cambridge, 2009).

[316] Y. Imry, Introduction to Mesoscopic Physics, 2nd ed. (Oxford University Press, USA, 2008).

[317] E. Boulat, H. Saleur, and P. Schmitteckert, Phys. Rev. Lett. 101, 140601 (2008).

[318] S. Kirino and K. Ueda, Journal of the Physical Society of Japan 79, 093710 (2010).

[319] S. Kirino, T. Fujii, J. Zhao, and K. Ueda, Journal of the Physical Society of Japan 77, 084704 (2008).

[320] D. Bohr and P. Schmitteckert, Phys. Rev. B 75, 241103 (2007).

[321] F. Heidrich-Meisner, I. González, K. A. Al-Hassanieh, A. E. Feiguin, M. J. Rozenberg, and E. Dagotto, Phys. Rev. B 82, 205110 (2010). 
[322] L. G. G. V. Dias da Silva, K. A. Al-Hassanieh, A. E. Feiguin, F. A. Reboredo, and E. Dagotto, Phys. Rev. B 81, 125113 (2010).

[323] F. Heidrich-Meisner, A. E. Feiguin, and E. Dagotto, Phys. Rev. B 79, 235336 (2009).

[324] L. G. G. V. Dias da Silva, F. Heidrich-Meisner, A. E. Feiguin, C. A. Büsser, G. B. Martins, E. V. Anda, and E. Dagotto, Phys. Rev. B 78, 195317 (2008).

[325] F. Krausz and M. Ivanov, Rev. Mod. Phys. 81, 163 (2009).

[326] P. Baum, D.-S. Yang, and A. H. Zewail, Science 318, 788 (2007).

[327] C. W. Siders, A. Cavalleri, K. Sokolowski-Tinten, C. Tth, T. Guo, M. Kammler, M. H. v. Hoegen, K. R. Wilson, D. v. d. Linde, and C. P. J. Barty, Science 286, 1340 (1999).

[328] E. Collet, M.-H. Leme-Cailleau, M. Buron-Le Cointe, H. Cailleau, M. Wulff, T. Luty, S.-Y. Koshihara, M. Meyer, L. Toupet, P. Rabiller, and S. Techert, Science 300, 612 (2003).

[329] K. Sokolowski-Tinten, C. Blome, J. Blums1, A. Cavalleri, C. Dietrich, A. Tarasevitch, I. Uschmann, E. Frster, M. Kammler, M. H. von Hoegen, and D. von der Linde, Nature 422, 287 (2003).

[330] S. Wall, D. Brida, S. R. Clark, H. Ehrke, D. Jaksch, A. Ardavan, S. Bonora, H. Uemura, Y. Takahashi, T. Hasegawa, H. Okamoto, G. Cerullo, and A. Cavalleri, Nature Physics 7, 114 (2011).

[331] J. C. Petersen, S. Kaiser, N. Dean, A. Simoncig, H. Y. Liu, A. L. Cavalieri, C. Cacho, I. C. E. Turcu, E. Springate, F. Frassetto, L. Poletto, S. S. Dhesi, H. Berger, and A. Cavalleri, Phys. Rev. Lett. 107, 177402 (2011).

[332] N. Dean, J. C. Petersen, D. Fausti, R. I. Tobey, S. Kaiser, L. V. Gasparov, H. Berger, and A. Cavalleri, Phys. Rev. Lett. 106, 016401 (2011).

[333] A. Cavalleri, T. Dekorsy, H. H. W. Chong, J. C. Kieffer, and R. W. Schoenlein, Phys. Rev. B 70, 161102 (2004).

[334] C. W. Siders and A. Cavalleri, Science 300, 591 (2003).

[335] A. Cavalleri, Science 318, 755 (2007).

[336] M. Gutzwiller, Chaos in Classical and Quantum Mechanics, Interdisciplinary Applied Mathematics (Springer, New York, 1990). 
[337] J. L. Lebowitz and O. Penrose, Physics Today 26, 155 (1973).

[338] J.-S. Caux and J. Mossel, Journal of Statistical Mechanics: Theory and Experiment 2011, P02023 (2011).

[339] M. Rigol, V. Dunjko, V. Yurovsky, and M. Olshanii, Phys. Rev. Lett. 98, 050405 (2007).

[340] C. Kollath, A. Läuchli, and E. Altman, Phys. Rev. Lett. 98, 180601 (2007).

[341] P. Barmettler, M. Punk, V. Gritsev, E. Demler, and E. Altman, Phys. Rev. Lett. 102, 130603 (2009).

[342] P. Barmettler, M. Punk, V. Gritsev, E. Demler, and E. Altman, New Journal of Physics 12, 055017 (2010).

[343] G. Roux, Phys. Rev. A 81, 053604 (2010).

[344] T. Barthel and U. Schollwöck, Phys. Rev. Lett. 100, 100601 (2008).

[345] M. Cramer, A. Flesch, I. P. McCulloch, U. Schollwöck, and J. Eisert, Phys. Rev. Lett. 101, 063001 (2008).

[346] A. Flesch, M. Cramer, I. P. McCulloch, U. Schollwöck, and J. Eisert, Phys. Rev. A 78, 033608 (2008).

[347] M. Eckstein and M. Kollar, Phys. Rev. Lett. 100, 120404 (2008).

[348] M. Rigol, V. Dunjko, and M. Olshanii, Nature 452, 854 (2008).

[349] J. Berges, S. Borsanyi, and C. Wetterich, Phys. Rev. Lett. 93, 142002 (2004).

[350] M. Moeckel and S. Kehrein, New Journal of Physics 12, 055016 (2010).

[351] M. Moeckel and S. Kehrein, Ann. Phys. 324, 2146 (2009).

[352] M. Moeckel and S. Kehrein, Phys. Rev. Lett. 100, 175702 (2008).

[353] M. Eckstein, M. Kollar, and P. Werner, Phys. Rev. Lett. 103, 056403 (2009).

[354] M. Srednicki, Phys. Rev. E 50, 888 (1994).

[355] M. Rigol, Phys. Rev. Lett. 103, 100403 (2009).

[356] M. Rigol and L. F. Santos, Phys. Rev. A 82, 011604 (2010).

[357] L. F. Santos and M. Rigol, Phys. Rev. E 82, 031130 (2010). 
[358] P. Jacquod and D. L. Shepelyansky, Phys. Rev. Lett. 79, 1837 (1997).

[359] E. M. Purcell and R. V. Pound, Phys. Rev. 81, 279 (1951).

[360] N. F. Ramsey, Phys. Rev. 103, 20 (1956).

[361] S. Braun, J. P. Ronzheimer, M. Schreiber, S. S. Hodgman, T. Rom, I. Bloch, and U. Schneider, Science 339, 52 (2013).

[362] L. D. Carr, Science 339, 42 (2013).

[363] S. Mandt, Physik Journal 12, 21 (2013).

[364] J. Dunkel and S. Hilbert, Nature Physics 10, 67 (2014).

[365] D. Frenkel and P. B. Warren, arXiv:1403.4299 (2014).

[366] U. Schneider, S. Mandt, A. Rapp, S. Braun, H. Weimer, I. Bloch, and A. Rosch, arXiv:1407.4127 (2014).

[367] J. Dunkel and S. Hilbert, arXiv:1403.6058 (2014).

[368] J. Dunkel and S. Hilbert, arXiv:1408.5392 (2014).

[369] S. Hilbert, P. Hänggi, and J. Dunkel, arXiv:1408.5382 (2014).

[370] R. H. Swendsen and J.-S. Wang, arXiv:1410.4619 (2014).

[371] M. Rigol, in Quantum Gases: Finite Temperatures and NonEquilibrium Dynamics, edited by N. Proukakis, S. Gardiner, and M. Davis (Imperial College Press, London, 2013).

[372] P. Calabrese and J. Cardy, Phys. Rev. Lett. 96, 136801 (2006).

[373] A. M. Läuchli and C. Kollath, J. Stat. Mech.: Theor. and Exp. P05018 (2008).

[374] M. Cheneau, P. Barmettler, D. Poletti, M. Endres, P. Schauß, T. Fukuhara, C. Gross, I. Bloch, C. Kollath, and S. Kuhr, Nature 481, $484(2012)$.

[375] T. W. B. Kibble, J. Phys. A 9, 1387 (1976).

[376] W. H. Zurek, Nature 317, 505 (1985).

[377] W. H. Zurek, Phys. Rep. 276, 177 (1996).

[378] N. W. Ashcroft and N. D. Mermin, Solid State Physics (Brooks/Cole, USA, 1976). 
[379] L. Xia, L. A. Zundel, J. Carrasquilla, A. Reinhard, J. M. Wilson, M. Rigol, and D. S. Weiss, arXiv:1409.2882 (2014).

[380] K. Winkler, G. Thalhammer, F. Lang, R. Grimm, J. H. Denschlag, A. J. Daley, A. Kantian, H. P. Büchler, and P. Zoller, Nature 441, 853 (2006).

[381] R. C.-G. P.G. Kevrekidis, D.J. Frantzeskakis, Emergent Nonlinear Phenomena in Bose-Einstein Condensates (Springer, Berlin-Heidelberg, 2008).

[382] L. D. Carr and J. Brand, Phys. Rev. Lett. 92, 040401 (2004).

[383] S. Burger, K. Bongs, S. Dettmer, W. Ertmer, K. Sengstock, A. Sanpera, G. V. Shlyapnikov, and M. Lewenstein, Phys. Rev. Lett. 83, 5198 (1999).

[384] J. Denschlag, J. E. Simsarian, D. L. Feder, C. W. Clark, L. A. Collins, J. Cubizolles, L. Deng, E. W. Hagley, K. Helmerson, W. P. Reinhardt, S. L. Rolston, B. I. Schneider, and W. D. Phillips, Science 287, 97 (2000)

[385] K. E. Strecker, G. B. Partridge, A. G. Truscott, and R. G. Hulet, Nature 417, 150 (2002).

[386] R. V. Mishmash and L. D. Carr, Phys. Rev. Lett. 103, 140403 (2009).

[387] R. V. Mishmash, I. Danshita, C. W. Clark, and L. D. Carr, Phys. Rev. A 80, 053612 (2009).

[388] F. Dalfovo, S. Giorgini, L. P. Pitaevskii, and S. Stringari, Rev. Mod. Phys. 71, 463 (1999).

[389] L. Pitaevskii and S. Stringari, Bose-Einstein Condensation (Oxford University Press, Oxford, 2003).

[390] R. Balakrishnan, I. I. Satija, and C. W. Clark, Phys. Rev. Lett. 103, 230403 (2009).

[391] S. Sachdev, K. Sengupta, and S. M. Girvin, Phys. Rev. B 66, 075128 (2002).

[392] J. Simon, W. S. Bakr, R. Ma, M. Tai, P. Preiss, and M. Greiner, Nature 472, 307 (2011). 